\title{
Universiteit
}

Leiden

The Netherlands

\section{Sensing at the surface of graphene field-effect transistors}

Fu, W.; Jiang, L.; Geest, E.P. van; Macedo Coelho Lima, L.; Schneider, G.F.

\section{Citation}

Fu, W., Jiang, L., Geest, E. P. van, Macedo Coelho Lima, L., \& Schneider, G. F. (2017). Sensing at the surface of graphene field-effect transistors. Advanced Materials, 29(6). doi:10.1002/adma.201603610

Version: $\quad$ Publisher's Version

License: $\quad$ Licensed under Article 25fa Copyright Act/Law (Amendment Taverne)

Downloaded from: https://hdl.handle.net/1887/3202698

Note: To cite this publication please use the final published version (if applicable). 


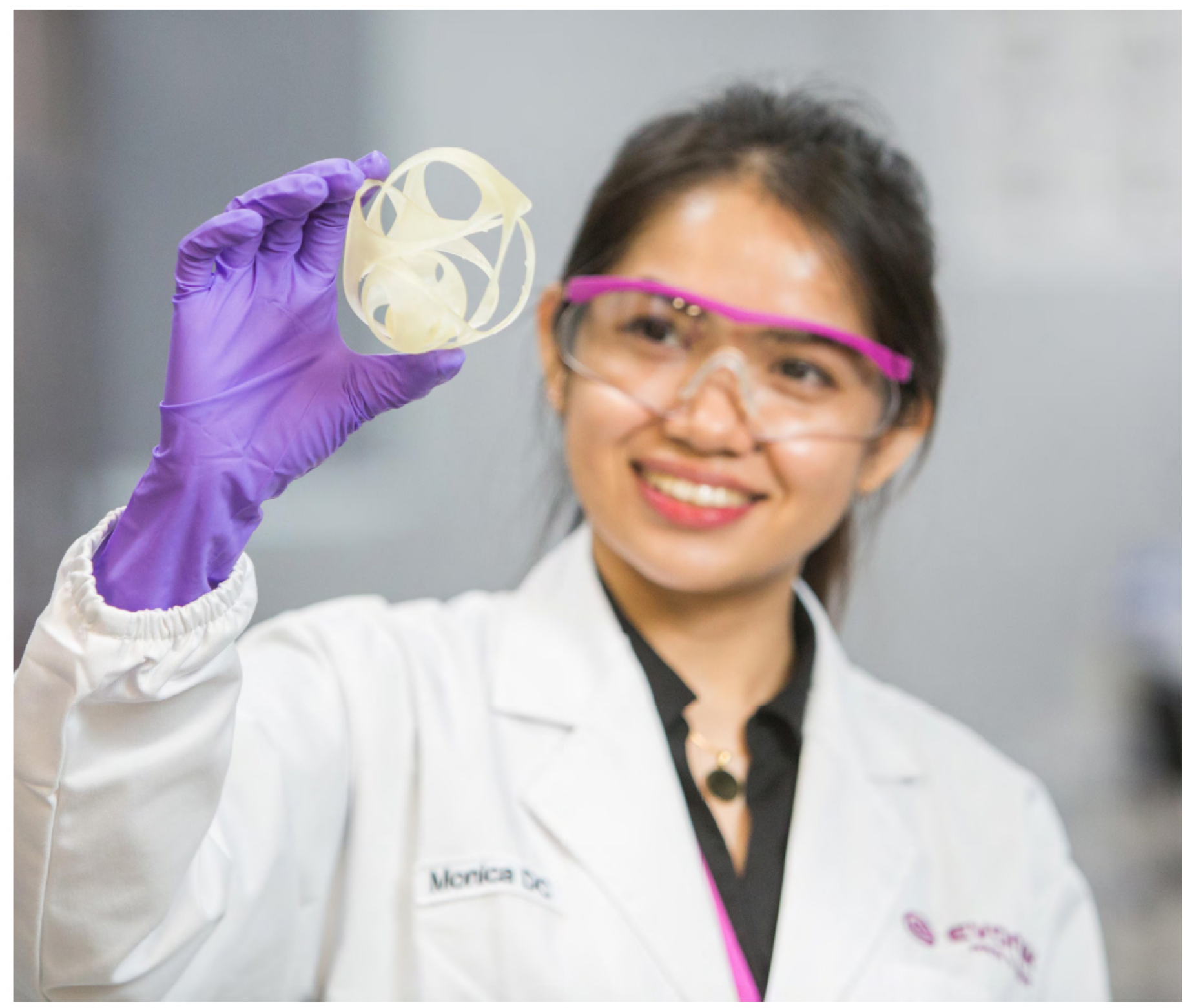

\section{Pushing the boundaries} of chemistry? It takes \#HumanChemistry

Make your curiosity and talent as a chemist matter to the world with a specialty chemicals leader. Together, we combine cutting-edge science with engineering expertise to create solutions that answer real-world problems. Find out how our approach to technology creates more opportunities for growth, and see what chemistry can do for you at:

evonik.com/career 


\title{
Sensing at the Surface of Graphene Field-Effect Transistors
}

\author{
Wangyang Fu, Lin Jiang, Erik P. van Geest, Lia M. C. Lima, and Grégory F. Schneider*
}

Recent research trends now offer new opportunities for developing the next generations of label-free biochemical sensors using graphene and other twodimensional materials. While the physics of graphene transistors operated in electrolyte is well grounded, important chemical challenges still remain to be addressed, namely the impact of the chemical functionalizations of graphene on the key electrical parameters and the sensing performances. In fact, graphene - at least ideal graphene - is highly chemically inert. The functionalizations and chemical alterations of the graphene surface - both covalently and non-covalently - are crucial steps that define the sensitivity of graphene. The presence, reactivity, adsorption of gas and ions, proteins, DNA, cells and tissues on graphene have been successfully monitored with graphene. This review aims to unify most of the work done so far on biochemical sensing at the surface of a (chemically functionalized) graphene field-effect transistor and the challenges that lie ahead. The authors are convinced that graphene biochemical sensors hold great promise to meet the ever-increasing demand for sensitivity, especially looking at the recent progresses suggesting that the obstacle of Debye screening can be overcome.

\section{Introduction: Challenges and Opportunities}

Ultrasensitive biosensors are opening up new opportunities for "personalized medicine" tailored to the specific biochemistry and diagnostic of individual patients. ${ }^{[1,2]}$ While versatile detection strategies exist, the main requirements for a biosensor is that the detection is sensitive (identification of clinically relevant concentrations of biomarkers in biological samples) and selective (availability of a suitable biological recognition element). ${ }^{[3]}$ Since the experimental preparation and observation of the electric field effect in graphene by the Manchester group in $2004,{ }^{[4]}$ biochemical sensing using graphene electronic devices has been actively pursued. ${ }^{[5-12]}$ The sensing principle roots on a change of the electrical conductance of the graphene channel upon adsorption of a molecule on the sensor surface. ${ }^{[5]}$ The uniqueness of graphene among other solid-state materials is that all carbon atoms are located on the surface, making the graphene surface potentially highly sensitive to any changes of its surrounding environment. Along with the excellent electrical properties of graphene, ${ }^{[13,14]}$ i.e., extraordinary high

Dr. W. Fu, L. Jiang, E. P. van Geest,

L. M. C. Lima, Dr. G. F. Schneider

Leiden University

Faculty of Science

Leiden Institute of Chemistry

Einsteinweg 55, 2333CC Leiden, The Netherlands

E-mail: g.f.schneider@chem.leidenuniv.nl

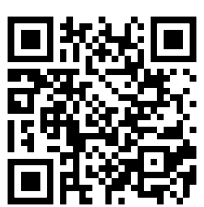

DOI: 10.1002/adma.201603610 mobility ${ }^{[15-17]}$ and low intrinsic electrical noise, ${ }^{[18-21]}$ graphene-based electronic biosensors demonstrated greater sensitivity than traditional bioassays. ${ }^{[22]}$ Additionally, graphene (at least ideal graphene) has a crystal lattice free of dangling bonds and is therefore intrinsically chemically inert. This inertness has been a driving force for the first attempts aiming at biointerfacing graphene with specific recognition moieties, via both covalent ${ }^{[23-28]}$ and noncovalent ${ }^{[29-32]}$ approaches, using different biochemical molecules and chemical treatments.

This article aims to provide a comprehensive overview and critical insight on biosensors using the surface of graphene as the sensing element. We evaluate the electronic and the chemical advantages of graphene, i.e., the high carrier mobility, low intrinsic electrical noise and the inert chemical properties, which are at the core of the sensing mechanisms but also crucial in applications where graphene must be interfaced with biological systems. Particularly, we highlight the importance of the chemistry of the graphene basal plane for sensing within the Debye screening length and shed light on the possibilities of sensing beyond the Debye screening.

\section{Physics of Graphene Field-effect Transistors (GFETs): the Basics for Sensing}

Graphene nanoelectronics provide a versatile platform for a wide spectrum of biochemical sensing applications. ${ }^{[33]}$ Detection can be realized through various mechanisms, including charge transfer, ${ }^{[34]}$ charge scattering, ${ }^{[35]}$ capacitive effect, ${ }^{[36]}$ and field effect. ${ }^{[6,7,37]}$ The field effect (i.e., the modulation of the electrical conductivity of a material upon the application of an external electric field, for example, induced by a charged biomolecule) has been widely regarded as the most reliable sensing mechanism. This effect has been harvested to design the first graphene field-effect transistor (GFET), ${ }^{[4]}$ which has inspired considerable experimental and theoretical work relating to the application of GFETs for high performance label-free chemical and biological sensors. ${ }^{[5-12,37]}$

\subsection{Back-gated GFETs}

The word transistor is a combination of two words: transfer and resistor. Usually a transistor is used to switch or amplify 
an electronic signal, comparable to a tap-valve that controls the supply and flow of water. Figure 1a depicts a back-gated GFET composed of a source/drain metallic electrode bridged together with a graphene channel. The carrier density, and thus the conductivity of the channel is typically modulated by the electric field by gating a highly conductive silicon substrate located underneath an insulating $\mathrm{SiO}_{2}$ dielectric layer to a range of voltages. As shown in Figure 1b, a typical measurement consists of applying a constant bias voltage, $V_{\text {sd }}$, between the source and the drain of the graphene channel, and monitor the resulting source-drain current $I_{\mathrm{sd}}$. A direct consequence of the electronic band structure of graphene ${ }^{[4,14]}$ is that graphene-based FET devices are of metallic nature and cannot be switched off at room temperature. Besides chemical modification, graphene nanoribbon, graphene nanomesh, and graphene nanoring, [38] have also been proved as rational designs of the graphene to open a bandgap, yielding an improved transistor $I_{\text {on }} / I_{\text {off }}$ ratio. Nevertheless, the transistor $I_{\text {on }} / I_{\text {off }}$ ratio has no direct relation to the performances of a sensor device, although it is related to graphene digital applications requiring high on state current $\left(I_{\text {on }}\right)$ and ultra-low power consumption at the off state $\left(I_{\text {off }}\right)$ of the transistors. By changing the back gate voltage $V_{\mathrm{g}}$, the electrochemical potential of the charge carriers (i.e., the Fermi energy) can be modulated. As a consequence, the type of charge carriers (which flow in the graphene channel and give the current $I_{\text {sd }}$ ) can continuously be tuned from holes (red curve in the left of Figure 1b) to electrons (gray curve in the right of Figure 1b), yielding a so-called "ambipolar behavior". At the transition between the electron and hole regime, the current is minimized and this point is also known as the charge neutrality point (CNP).

\subsection{Liquid-gated GFETs: Operation and Sensing Principle}

A change in the electric field can either be achieved using the above discussed back-gate voltage or be induced by physisorption or chemisorption of the target molecules. When the backgate is held at a fixed voltage the change in current between the drain and source thus can be ascribed to molecules adsorbed on the graphene surface, as demonstrated in a pioneering study by the Manchester group in 2007 with single molecule detection capability upon $\mathrm{NO}_{2}$ chemoadsorption. ${ }^{[5]}$

In contrast to the back-gate geometry, in a liquid-gated configuration the gate voltage is applied to the electrolyte via a reference electrode (Figure 1c). The reference electrode is coupled to the graphene channel through an interfacial capacitance $C$, consisting of a series of two capacitances, ${ }^{[37]}$ namely the quantum capacitance of graphene $\left(C_{\mathrm{Q}}\right),{ }^{[39]}$ and the double layer capacitance of the electrolyte $\left(C_{\mathrm{DL}}\right) \cdot{ }^{[40]}$ The double layer capacitor is a virtual capacitor formed by the separated charges located at the solid side and the solution side of the interface as described by the Poisson-Boltzmann equation. ${ }^{[41]}$ Liquid-gated GFET biosensors belong to the large family of ion-sensitive FETs, the first new concept of which was investigated by Bergveld with Si devices. ${ }^{[42,43]}$ Although the choice of the channel materials, the reference electrode, the operational mode, and the final encapsulation for liquid handling, vary from case to case, the heart of any ion-sensitive FETs lies on the interface

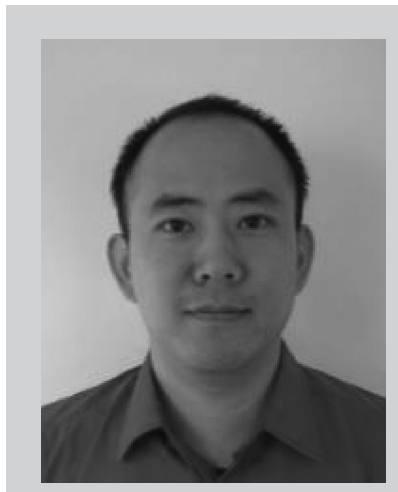

Wangyang Fu received his doctoral degree in physics from the Institute of Physics, Chinese Academy of Sciences in 2009. Currently, Dr. Fu is a postdoctoral researcher in the group of Dr. Schneider at Leiden University and recipient of a Veni grant (NWO) and an APM grant (SNF) for young researchers. Prior to joining the Leiden group in 2015, he was a postdoctoral researcher at University of Basel with Prof. Christian Schönenberger and at the Jülich Research Center with Prof. Andreas Offenhäusser (Humboldt, $\mathrm{AvH}$ ). His research interests focus on graphene nanoelectronics for biochemical sensing.

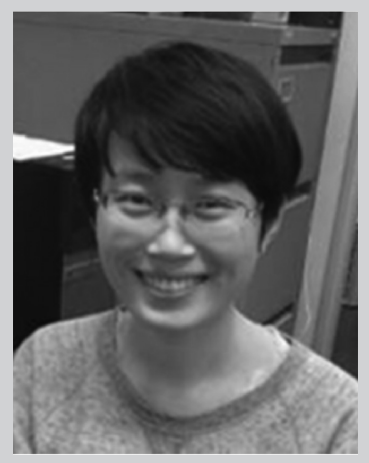

Lin Jiang is a PhD student in the group of Dr. Schneider at Leiden University. She received her MSc degree in chemistry from Shanghai University, China. Her current research interests focus on graphene defects generation for chemical (electrochemical) biosensing applications.

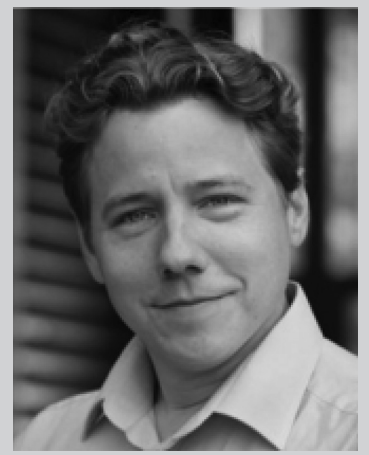

Grégory F. Schneider received his $\mathrm{PhD}$ in chemistry from the University of Strasbourg in 2005. Grégory is currently principal investigator and tenure track assistant professor of chemistry at the Leiden Institute of Chemistry. He received in 2014 an ERC starting grant and a Vidi grant from NWO to carry out chemical and biological research with graphene. Prior to joining the Leiden faculty in 2013 , he was a postdoctoral researcher at Harvard University with Prof. George Whitesides and at TU Delft with Prof. Cees Dekker. His research interests include nanotechnology, bionanotechnology, surface and interfacial chemistry, physical and organic chemistry, materials science, biophysical chemistry, nanofluidics, and self-assembly.

between the electrolyte and the solid FET materials. In general, GFETs are operated at low electrolyte gate voltage such that any electrochemical processes and exchange ionic currents are negligible, i.e., the interface is considered to be inert and purely capacitive, although this assumption is not always 
(a)

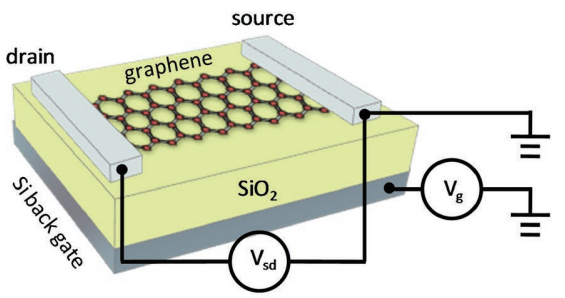

(c)

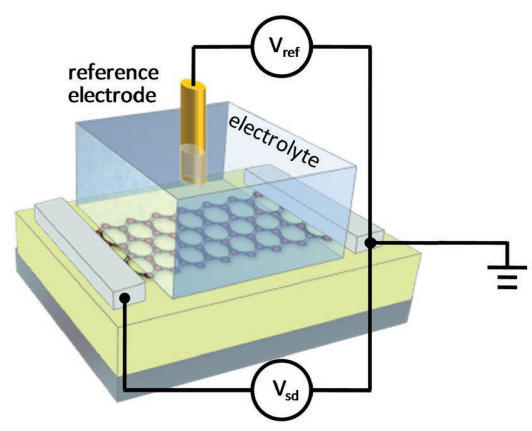

(b)

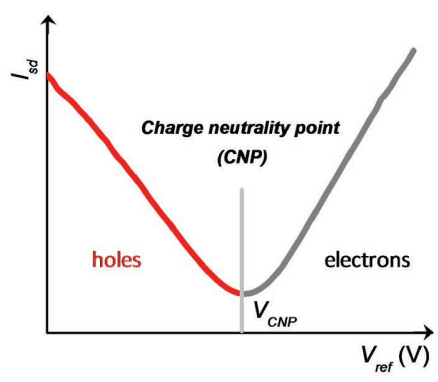

(d)

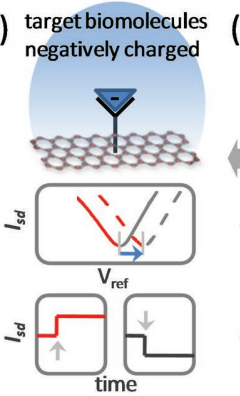

(e)

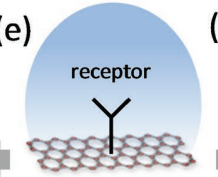

(f) target biomolecules positively charged
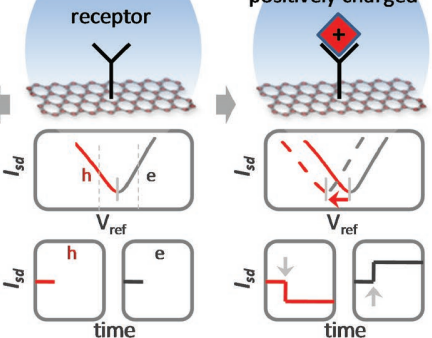

Figure 1. Working principle of a graphene field-effect transistor (GFET). a) Schematic of a back-gated GFET. b) Typical ambipolar transfer characteristics showing that the type of carriers in graphene can continuously be modulated from holes (on the left, in red) to electrons (on the right, in gray) using the field effect. The charge-neutrality point (CNP) is located at the transition between the electron and hole regime, where the current is minimized. c) Schematic of a liquid-gated GFET biosensor and its sensing principle (d-f). In the upper panel of (e), a receptor molecule is immobilized on the graphene surface. The plots of $I_{\text {sd }}$ versus $V_{\text {ref }}$ and $I_{\text {sd }}$ versus the time $t$ are shown in the middle and lower panels, respectively. The abbreviation ' $h$ ' in red refers a measurement carried in the hole regime and 'e' for the electron regime in gray. f) (respectively d) depicts the field effect resulting from the binding of positively (respectively negatively) charged target biomolecule on the receptor (as indicated by the gray arrows in the $I_{s d}(t)$ curves). The binding of a charged biomolecule as indicated by the blue arrows yields a shift in the curves of $I_{\text {sd }}$ versus $V_{\text {ref }}$.

explicitly stated in most of the literature. Experimental artifacts at moderate or relatively high electrolyte gate voltages resulting from such simple assumption are considered mainly of electrochemical nature that will be separately discussed in Section 4.4: Graphene-based electrochemical (GEC) biosensors.

The working principle of a liquid-gated GFET biosensor is illustrated in Figure 1d-f. In practice, liquid-gated GFETs can be integrated into microfluidic systems: ${ }^{[22]}$ the confinement into the fluidic channel helps in bringing the analyte to the sensor surface. ${ }^{[4]}$ In a typical measurement, receptor molecules are immobilized on the surface for selective recognition of target biomolecules (Figure 1e, upper panel). The corresponding $I_{\mathrm{sd}}$ versus $V_{\text {ref }}$ curve of such a liquid-gated GFET is shown in the middle panel (Figure 1e) with similar characteristics as the one observed for a back-gated GFET (Figure 1b). The lower panel of Figure 1e depicts the time dependent current $I_{\text {sd }}$ at a fixed reference potential $V_{\text {ref }}$ (as indicated by the dashed gray lines). In either the hole regime (as indicated by " $h$ ") or in the electron regime ('e'), when a positively charged target binds (Figure 1f, upper panel), a depletion of hole carriers (respectively an accumulation of electron carriers) in the graphene occurs due to the field effect. Such doping effect causes a negative shift of the $I_{\mathrm{sd}}\left(V_{\text {ref }}\right)$ curve as indicated by the blue arrow in Figure $1 \mathrm{f}$ (middle panel).

In the time-dependent measurement (i.e., the lower panel of Figure 1f), the binding of a positively charged molecule causes a decrease of the current $I_{\text {sd }}$ in the hole regime, and an increase of the current in the electron regime. Conversely, the binding of a negatively charged molecule (Figure 1e) induces a positive shift of the $I_{\text {sd }}\left(V_{\text {ref }}\right)$ curve and an increase in the $I_{\text {sd }}$ in the hole regime. In the electron regime - instead - the same event induces a negative shift of the $I_{\text {sd }}\left(V_{\text {ref }}\right)$ curve and a decrease of the current $I_{\text {sd }}$. This current modulation in the graphene channel can be expressed as a function of the change in the carrier density $\Delta n$, which is induced by and is proportional to the total number $N$ of charged biomolecules adsorbing on the graphene surface: ${ }^{[45]}$

$$
\Delta I_{\mathrm{sd}}=\frac{w}{l} V_{\mathrm{sd}} e \mu \Delta n \propto N
$$

where $w$ and $l$ are the width and length of the graphene channel, respectively, $e$ is the electron charge, and $\mu$ is the charge carrier mobility. In Equation (1), it is clear that the sensing response of a transistor sensor should be proportional to the total number of adsorbed biomolecules $N$. The quantitative monitoring of biomolecules, however, is non-trivial. Challenges lie in characterizing the number of charges each biomolecules carry, in controlling the chemical functionalization, and in identifying the exact sensing reactions at the graphene surface in each different regimes. We would also like to note here that, in principle, non-charged molecules should have no influences on the field-effect sensing response of GFET sensors, unless they can induce a charge variation (for example, through subtle dipole fluctuation ${ }^{[46]}$ or molecular engineering $\left.{ }^{[47]}\right)$. To deduce Equation (1), we assume that graphene has a constant carrier 
mobility $\mu$ upon the adsorption of biomolecules. This assumption is correct in most cases where the adsorbed biomolecules bind to the receptors and interact weakly with the graphene lattice. However, biomolecules that directly bind on a graphene surface form additional scattering centers, resulting in a change of the mobility of charge carriers. ${ }^{[35]}$ Additionally, practical sensor designs also take into account the changes in interfacial capacitance upon biomolecules adsorption. ${ }^{[36]}$

\subsection{Sensing with Graphene of High Carrier Mobility}

The change of the electrical current $\Delta I_{\text {sd }}$ resulting from the minute field-effect induced - for example - by the interaction of a biochemical molecule carrying an electron charge $e$, defines the sensing response $S=\Delta I_{\text {sd }} / N$. According to Equation (1), $S$ is therefore proportional to the mobility $\mu$ of graphene. With other parameters equal (especially the electrical noise performance), a higher sensing response $S$ implies a better sensor performance.

Because the performance of GFET sensors depends on the mobility $\mu$, the use and integration of high quality graphene into devices is preferential. To achieve high-quality pristine monolayer or few layer graphene sheets, the most commonly used method is the micromechanical cleavage of graphite with adhesive tape. ${ }^{[4]}$ This so-called 'scotch tape' technique involves splitting few layers of graphene from multi-layered graphite, after which the flakes are pressed and "dry-deposited" onto a silicon wafer. Compared to graphene synthesized using other methods, micromechanical cleavage yields graphene with higher mobility and lower intrinsic electrical noise, primarily because fewer structural defects are introduced upon preparation. ${ }^{[48]}$ Generally, for exfoliated graphene on $\mathrm{SiO}_{2} / \mathrm{Si}$ wafers, mobilities on the order of $\approx 3000-15000 \mathrm{~cm}^{2} \mathrm{~V}^{-1} \mathrm{~s}^{-1}$ are reported, ${ }^{[49]}$ which is more than one order of magnitude higher than those of silicon materials $\left(\approx 100-1500 \mathrm{~cm}^{2} \mathrm{~V}^{-1} \mathrm{~s}^{-1}\right) \cdot{ }^{[1,50]}$ The mobilities of the first graphene-based gas sensor devices were $\approx 5000 \mathrm{~cm}^{2} \mathrm{~V}^{-1} \mathrm{~s}^{-1}$.[5] Nowadays, at room temperature, carrier mobility up to 100000 $197600 \mathrm{~cm}^{2} \mathrm{~V}^{-1} \mathrm{~s}^{-1}$, can be achieved by encapsulating graphene in boron nitride $(\mathrm{BN}),{ }^{[17,51,52]}$ providing unprecedented possibilities for sensing applications. The fact that this idea has only been realized very recently (with h-BN capped $\mathrm{MoS}_{2}{ }^{[53]}$ ) is not a surprise: groups that work on high quality BN coated graphene samples, very often focus on cryogenic measurements of the physics of the 2D electron gases in graphene rather than its biological sensing applications; moreover, the fabrication methods are very delicate (it is not yet trivial to achieve an ideal interface) and the lack of scalability is still an important drawback. ${ }^{[17]}$

Despite all the impressive achievements in the electrical performances of graphene devices, the reproducibility and homogeneity of sample preparation and the relatively small size (on micrometer scale) represent the bottleneck for using exfoliated graphene for practical applications. Larger sheets of few-layer or monolayer graphene can now be directly synthesized via chemical vapor deposition (CVD) on nickel or copper substrates ${ }^{[54,55]}$ with mobilities rivaling the ones of exfoliated samples. ${ }^{[56]}$ For samples placed on $\mathrm{SiO}_{2} / \mathrm{Si}$ wafers, mobilities on the order of $\approx 1000-10000 \mathrm{~cm}^{2} \mathrm{~V}^{-1} \mathrm{~s}^{-1}$ are now routinely observed and regarded as the standard for graphene transistor products for biochemical sensing applications. ${ }^{[57]}$ The electronic performances of CVD graphene ${ }^{[58]}$ can be significantly enhanced by growing single-crystal graphene free of grain boundaries ${ }^{[59]}$ and by using a BN substrate similarly to exfoliated graphene, with which mobility up to $\approx 50000-350000 \mathrm{~cm}^{2} \mathrm{~V}^{-1} \mathrm{~s}^{-1}$ can be achieved. ${ }^{60,61]}$ These mobility numbers are rivaling those of exfoliated samples, making the CVD process ideal for large-area synthesis of high-quality and uniform graphene for sensing applications.

\subsection{Electrical Noise Performances of Graphene Materials}

At low frequencies ( $\lesssim 100 \mathrm{~Hz}$ ), the ubiquitous $1 / f$ noise, whose power spectral density (PSD) spectrum inversely depends on the frequency $f{ }^{[62]}$ seriously impedes the sensing performances of GFET. ${ }^{[18]}$ This low-frequency $1 / f$ noise is even more pronounced for devices that are scaled down to nanometer dimensions, where the channel current becomes more prone to fluctuations due to, particularly, interface and surface trap states. ${ }^{[63,64]}$ It is the level of these unwanted fluctuations (along with the sensing response $S$ ) that determines the ultimate detection limit of GFET biosensors. The $1 / f$ noise of graphene monolayers supported on a substrate is comparable to that of bulk semiconductors (including Si). ${ }^{[19]}$ For freestanding or bilayer graphene, however, the $1 / f$ noise was found to be one order of magnitude lower through the effective screening of potential fluctuations from external charged impurities (for example, oxide traps or interface states).${ }^{[19,21]}$ The fact that graphene possesses both superior mobility and noise performances, gives it a better signal-to-noise ratio (SNR) as advocated from time to time by literature, reporting graphene based biochemical sensors with superior performances compared to their Si based counterpart devices. ${ }^{[65-68]}$ Figure $2 \mathrm{~b}$ compares the noise performances of a GFET device supported by a $\mathrm{SiO}_{2} / \mathrm{Si}$ substrate and its counterpart after suspending the graphene monolayer by etching the underlying $\mathrm{SiO}_{2}$ substrate (Figure 2a). ${ }^{[21]}$ The large noise suppression was mainly attributed to the removal of any external trap states in the supported $\mathrm{SiO}_{2}$ substrate since the $1 / f$ noise in graphene devices is a surface phenomenon. ${ }^{[64]}$

Similarly, defects in the graphene are another source of noise. For example, the permanent oxygen-based defects contained in graphene oxide $(\mathrm{GO})$ or reduced graphene oxide (rGO) - introduced by over-oxidation (for GO) or incomplete removal of oxygen groups (for $\mathrm{rGO}$ ) - lead to inferior electrical quality (i.e., degradation in the mobility and noise performance) compared to scotch-tape or CVD graphene. ${ }^{[25]}$ Interestingly, environmental exposure and aging of graphene devices also increase the level of noise, suggesting that a proper capping layer or surface functionalization may circumvent an increase of noise. ${ }^{[69]}$ Indeed, by encapsulating a single layer graphene between two layers of hexagonal boron nitride (h-BN, as shown in Figure 2c), the noise spectral density normalized to the channel area (lower dots in red, Figure 2d) can be suppressed up to one order of magnitude lower compared to non-encapsulated devices on $\mathrm{Si} / \mathrm{SiO}_{2}$ (upper squares in blue, Figure 2d). ${ }^{[70]}$ In the case of silicon FET, the functionalization of the sensor channel (in this case a silicon nanowire buried in a $\mathrm{SiO}_{2}$ dielectric) with 3-aminopropyl-triethoxysilane (APTES) yields better noise performances (up to 60 times), presumably 
(a)

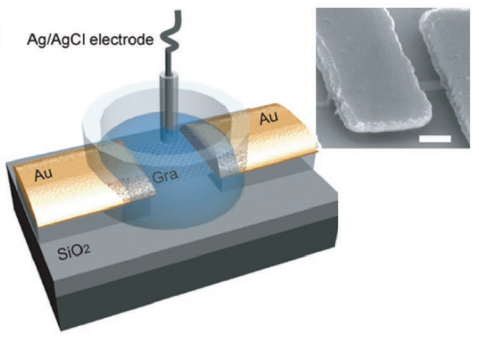

(c)

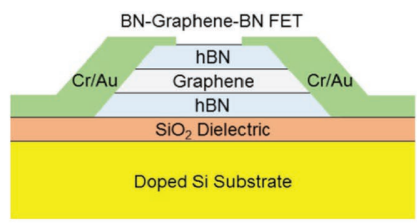

(e)

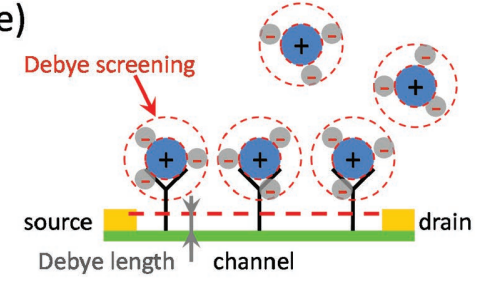

(b)

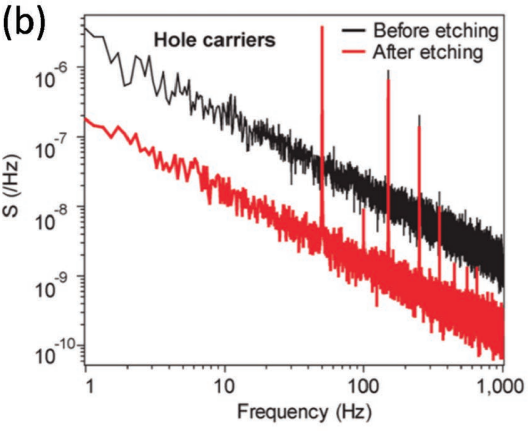

(d)

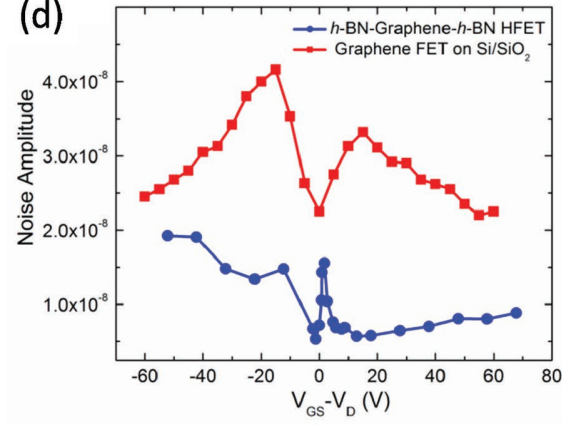

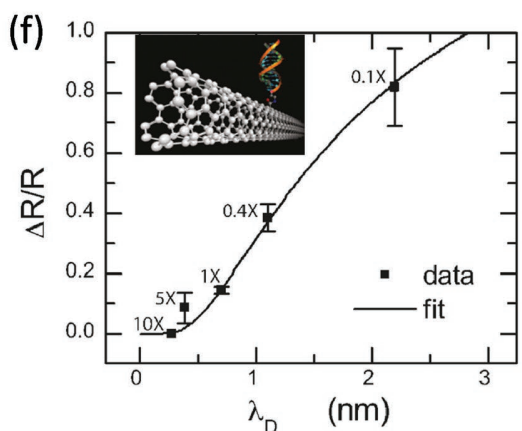

Figure 2. a) Schematic representation of the experimental setup where a single-layer graphene is supported in solution by $\mathrm{Cr} / \mathrm{Au}$ contacts to bridge a trench in the oxide. The inset shows a SEM image of a suspended graphene device. Scale bar is $0.5 \mu \mathrm{m}$. b) Comparison of graphene's noise power spectra in the linear operating modes with holes as carriers before (upper curve in black) and after suspension of the graphene layer (lower curve in red). The spikes are due to $50 \mathrm{~Hz}$ noise coupled from the power lines. Reproduced with permission. ${ }^{[21]}$ Copyright 2010 , American Chemical Society. c) Schematics of BN-graphene-BN FET. d) Noise amplitude as a function of the gate voltage for both BN-graphene-BN FET (lower dots in blue) and conventional non-encapsulated GFET on $\mathrm{Si} / \mathrm{SiO}_{2}$ wafer (upper squares in red). Reproduced with permission. ${ }^{70]}$ Copyright 2015, AIP Publishing LLC. e) The biomolecules carry zero net charge due to the Debye screening effect of ions in electrolyte. $\mathrm{f})$ Relative changes in resistance ( $\Delta R / R)$ of a carbon nanotube transistor versus buffer concentration. Increasing the buffer concentration will reduce the Debye length ( $\left.\lambda_{D}\right)$ so that most of the DNA's negative charge will be screened by counter ions in the electrolyte, resulting in a decreased sensing response ( $\Delta R / R)$. Inset: schematic representation of a DNA molecule binds on the sidewall of a carbon nanotube. Reproduced with permission. ${ }^{\text {72] }}$ Copyright 2010, American Chemical Society.

due to the passivation of the oxide traps and interface states at the sensor surface. ${ }^{[71]}$ On the contrary, for carbon nanotubes, a two-level random telegraphic noise (RTN) was reported and ascribed to a single probe molecule (more precisely, the binding and unbinding of charged target biomolecules at its active sites), which was covalently bound to a defect in the carbon nanotube sidewall. ${ }^{[72]}$ A suppression of the RTN was observed in high ionic strength buffer solutions (ionic screening) and for high gate potentials (when the target biomolecules are repelled from the nanotube). The influence of surface functionalization on the noise performances of liquid-gated GFETs has not yet been systematically studied. As we described in the previous Section 2.3, with other parameters equal (especially the electrical noise performance), a higher mobility implies a better sensor performance when considering the adsorption of charged biomolecules. We would like to note here that a higher mobility also complies with graphene bearing less defects and impurities, which is in favor of an improved noise performance (although there is still not enough experimental evidences or theories that could directly and unambiguously link the high mobility of GFETs to their noise performances).

\subsection{Debye Screening}

The true potential of graphene sensors in physiological solutions (and electrical sensors in general) is still behind expectations. This is because that GFETs are primarily sensitive to the charges carried by the biomolecules adsorbed on graphene surface, which suffer from the ionic screening due to mobile ions present in the solution, known as Debye screening effect. ${ }^{[73]}$ In electrolytes, this screening effect is characterized by the Debye length, which is the measure of how far a charge carrier's net electrostatic effect persists, outside of which charges are effectively screened and only $36.8 \%(1 / e, e=2.72)$ of the charges can still be seen by the graphene sensing devices. This screening layer (or diffuse layer) is composed of movable ions attracted to a charged surface via the Coulomb force (Figure 2e). The Debye screening effect is an intrinsic thermodynamic property of large systems of mobile charges. In the following we will examine the Debye screening dilemma in details and look into recent progresses.

For aqueous solutions at room temperature, the Debye length (in unit of nanometer) is given by: ${ }^{[7]} \lambda_{D}=0.304 / I^{1 / 2}$, where $I$ is the ionic strength expressed in $\mathrm{mol} / \mathrm{L}(\mathrm{M})$, and is typically $\approx 0.7 \mathrm{~nm}$ in physiological conditions. Given the typical several nanometer size of biomolecules, it is therefore likely only small - or even no net electrostatic effect - can be recorded by the transistor (see Figure 2e). In Table 1, we have summarized the sensing responses of several ion-sensitive FETs (including nanowire, nanotube and graphene ion-sensitive FETs) at different salt concentrations and biomolecule-to-sensor distances. Indeed, under physiological conditions of $1 \times \mathrm{PBS}$ $\left(\lambda_{D} \approx 0.7 \mathrm{~nm}\right.$ ) and near side distance of $\approx 1 \mathrm{~nm}$ (for example for 
Table 1. Selected summary of Debye screening length limitation.

\begin{tabular}{|c|c|c|c|c|c|}
\hline \multirow{2}{*}{$\begin{array}{l}\text { Surface modification + Target biomolecules } \\
\text { Biotin + Streptavidin }\end{array}$} & \multirow{2}{*}{$\begin{array}{l}\text { Distancel) }^{\mathrm{l}} \\
\quad \approx 1 \mathrm{~nm}\end{array}$} & \multirow{2}{*}{$\begin{array}{l}\left.\text { Debye length } \lambda_{\mathrm{D}} \text { (buffers }{ }^{11)}\right) \\
\quad \approx 7 \mathrm{~nm}(0.01 \times \mathrm{PBS})\end{array}$} & \multicolumn{2}{|c|}{$\begin{array}{c}\text { Concentration \& relative sensitivity } \\
{[\quad[R / R]}\end{array}$} & \multirow{2}{*}{$\begin{array}{c}\text { Refs (comments) } \\
{ }^{[74] \text { Nanowire }}\end{array}$} \\
\hline & & & $10 \mathrm{nM}$ & $\approx 15 \%$ & \\
\hline & & $\approx 0.7 \mathrm{~nm}(1 \times \mathrm{PBS})$ & & $\approx 0 \%$ & \\
\hline \multirow[t]{4}{*}{ ssDNA + ssDNA (complementary) } & At surface & $\approx 3 \mathrm{~nm}(0.05 \times \mathrm{PBS})$ & $10 \mathrm{pM}$ & $\approx 15-40 \%$ & ${ }^{[74]}$ Nanowire \\
\hline & $\approx 1.4 \mathrm{~nm}$ & $\approx 2 \mathrm{~nm}(0.1 \times \mathrm{PBS})$ & $1 \mu \mathrm{M}$ & $\approx 80 \%$ & ${ }^{[72]}$ Nanotube \\
\hline & & $\approx 0.7 \mathrm{~nm}(1 \times \mathrm{PBS})$ & & $\approx 12 \%$ & \\
\hline & & $\approx 0.2 \mathrm{~nm}(10 \times \mathrm{PBS})$ & & $\approx 0 \%$ & \\
\hline \multirow[t]{2}{*}{$P N A+s s D N A$} & $2.6 \mathrm{~nm}$ (fully complementary) & $\approx 10 \mathrm{~nm}(0.01 \times \mathrm{SSC})$ & $1 \mathrm{pM}(1 \mathrm{nM})$ & $\approx 19 \%(\approx 51 \%)$ & ${ }^{[75]}$ Nanowire \\
\hline & $7.7 \mathrm{~nm}$ (noncomplementary) & & $1 \mathrm{nM}$ & $\approx 0 \%$ & \\
\hline \multirow[t]{3}{*}{ APTES ${ }^{(I I)}+P S A^{(\mathrm{V})}$} & $\approx 0.8 \mathrm{~nm}$ & $\approx 7 \mathrm{~nm}(1 \mathrm{mM} \mathrm{PB})$ & $100 \mathrm{nM}$ & $\approx 112 \mathrm{mV*}$ & ${ }^{[76]}$ Nanowire \\
\hline & & $\approx 2 \mathrm{~nm}(10 \mathrm{mM}$ PB) & & $\approx 8 \mathrm{mV} *$ & \\
\hline & & $\approx 1 \mathrm{~nm}(50 \mathrm{mM} \mathrm{PB})$ & & $\approx 0$ & \\
\hline Bare graphene $+B S A^{V}$ ) (nonspecific) & At surface & $\approx 2 \mathrm{~nm}(10 \mathrm{mM}$ PBS $)$ & $300 \mathrm{pM}$ & $\approx 2 \%$ & ${ }^{[10]}$ Graphene \\
\hline 20-mer DNA aptamer + ATPVII) & $<2.6 \mathrm{~nm}$ & $\approx 2-3 \mathrm{~nm}(5-10 \mathrm{mM}$ PB) & $10 \mathrm{pM}$ & $\approx 1 \%$ & ${ }^{[77]}$ Graphene \\
\hline \multirow[t]{3}{*}{ PSA monoclonal antibody $+P S A^{(V)}$} & $<15 \mathrm{~nm}$ & $\approx 70 \mathrm{~nm}(1 \mu \mathrm{M}$ PBS $)$ & $\approx 1 \mathrm{nM}$ & $\approx 17 \%$ & ${ }^{[78]}$ Graphene \\
\hline & & & $\approx 1 \mathrm{pM}$ & $\approx 12 \%$ & \\
\hline & & & $\approx 1 \mathrm{fM}$ & $\approx 2 \%$ & \\
\hline
\end{tabular}

1) Near side distance of the target biomolecules from the device surface; "I)Please refer to the according literature for the exact background ionic strength and $\mathrm{pH}$ value of the buffer solutions; ${ }^{\text {III) }}$ (3-aminopropyl)-triethoxysilane; ${ }^{\mathrm{IV})}$ Prostate specific antigen, prostate cancer biomarker; ${ }^{\mathrm{V})}$ Bovine serum albumin; ${ }^{\mathrm{VI})}$ Adenosine triphosphate.

"Relative changes not given.

N.B.: $T=293 \mathrm{~K}$ unless stated otherwise

biotin receptors anchored on the transistor surface), nanowire ion-sensitive FETs showed no response upon the binding of streptavidin from a $10 \mathrm{nM}$ solution. ${ }^{[74]}$ Even at low salt concentrations, the sensing response upon hybridization of complementary DNA molecules (i.e., the normalized resistance change) was found to decrease dramatically from $80 \%$ to $12 \%$ by increasing the buffer concentration from $0.1 \times \mathrm{PBS}$ to $1 \times \mathrm{PBS}$ in a manner that follows the Debye length considerations (as given by the fitting line in Figure 2f). ${ }^{\text {[2] }}$ Increasing further the buffer concentration to $10 \times \mathrm{PBS}$ resulted in a full screening of the biological binding signal even at a relatively high DNA concentration of $1 \mu \mathrm{M}$.

The Debye screening effect has put a fundamental limit to the possible applications of the graphene ion-sensitive FETs (and ion-sensitive FETs in general) for biosensing applications, although ion-sensitive FETs can, in principle, be sensitive to changes below one single charge. ${ }^{[5,79]}$ There is significant evidence in the literature that the sensing performances can be improved by circumventing the Debye screening effect, for example by designing short antibodies, by performing ex situ measurement in low ionic strength buffers, and by incorporating porous polymer layers permeable to biomolecules (Table 1). ${ }^{[74-77,80]}$ At the end of this article, we will discuss in detail recent progress on operating GFETs at high frequencies which suggests that Debye screening can be overcome: ${ }^{[46]} 1$. without any special design or engineering of the receptor molecules and the sensor environments, and 2. in physiological conditions to facilitate in situ, real-time biosensing.

\section{Meeting the Challenges in Chemical Functionalization of Graphene for Biochemical Sensing}

Due to its large aromatic $\mathrm{sp}^{2}$ carbon lattice, free of dangling bonds, graphene is intrinsically chemically inert. ${ }^{[12]}$ The broad sensing potential of graphene can only be unlocked by the introduction of sensitizer (bio)molecules and structures, e.g., various inorganic groups, ${ }^{[23-25,81-90]}$ organic or organometallic molecules, ${ }^{[37,91-96]}$ DNAs, ${ }^{[97-101]}$ proteins, ${ }^{[102]}$ peptides, ${ }^{[30,31,103,104]}$ nanoparticles, ${ }^{[105-107]}$ and 2D heterostructures. ${ }^{[51,52,61,108]}$ These molecules are able to respond chemically or physically to their nearby environment, whose responses could then be transduced into an appreciable change in the conductivity of the carbonbased honeycomb scaffold. The introduction of such chemical moieties on the graphene surface or edge is often referred to as graphene functionalization. ${ }^{[109,110]}$ Chemical functionalization of graphene is commonly achieved using either covalent ${ }^{[23-28]}$ or non-covalent ${ }^{[29-32]}$ strategies. The resulting graphene materials contain specific recognition moieties for biochemical sensing, but still share, to a large extent, the same carbon honeycomb backbone and the electrical properties, especially the field effect, of graphene. Here, we generally include all the developed electronic biochemical sensors based on graphene and functionalized graphene. The physics of GFETs described in Section 2 can serve as the basics for sensing of (functionalized) graphene in general. A selected list of frequently used graphene surface chemistry (and their influences on the electrical properties of graphene) is presented in Table 2. Typically, 
Table 2. Selected examples of frequently used graphene surface modifications.

\begin{tabular}{|c|c|c|c|c|c|c|}
\hline \multirow[t]{2}{*}{ Surface modification } & \multirow[t]{2}{*}{ Functional group } & \multicolumn{2}{|c|}{ Degree of functionalization } & \multicolumn{2}{|c|}{ Device performance (mobility) } & \multirow[t]{2}{*}{ Refs (comments) } \\
\hline & & Unmodified & Modified & Pristine $\left[\mathrm{cm}^{2} \mathrm{~V}^{-1} \mathrm{~s}^{-1}\right]$ & Func.ed $\left[\mathrm{cm}^{2} \mathrm{~V}^{-1} \mathrm{~s}^{-1}\right]$ & \\
\hline \multirow[t]{3}{*}{ Hydrogenation') (Graphane) } & $\mathrm{H}$ & - & - & $\approx 14000(40-160 \mathrm{~K})$ & down to $\approx 10$ at low $T$ & ${ }^{[23]}$ Exfoliation \\
\hline & $\mathrm{H}$ & $\mathrm{I}_{D} / \mathrm{I}_{G}=0.1^{\mathrm{a})}$ & $\mathrm{I}_{\mathrm{D}} / \mathrm{I}_{\mathrm{G}}=1.43^{\mathrm{a})}$ & 1100 & 149 & ${ }^{[82]} \mathrm{CVD}$ \\
\hline & & & $\mathrm{I}_{\mathrm{D}} / \mathrm{I}_{\mathrm{G}}=2.05^{\mathrm{a})}$ & & 59 & \\
\hline \multirow[t]{2}{*}{ Fluorination') } & $\mathrm{F}$ & $\mathrm{I}_{\mathrm{D}} / \mathrm{I}_{\mathrm{G}}=0^{\mathrm{a})}$ & $\mathrm{I}_{\mathrm{D}} / \mathrm{I}_{\mathrm{G}}=3.8^{\mathrm{a})}$ & - & $\approx 150$ & ${ }^{[83]}$ Exfoliation \\
\hline & $\mathrm{F}$ & - & $\left.F / C: 0.21-0.25^{b}\right)$ & - & Insulator & ${ }^{[84]} \mathrm{CVD}$ (3-10 layers) \\
\hline \multirow[t]{2}{*}{ (hlorination') } & $\mathrm{Cl}$ & - & $\mathrm{wt} \% \mathrm{Cl}=42.6$ & 2076 & 1535 & ${ }^{[85]} \mathrm{CVD}$ \\
\hline & $\mathrm{Cl}$ & - & $\mathrm{wt} \% \mathrm{Cl}=20.5$ & 5000 & 1 & ${ }^{[86]} \mathrm{CVD}$ \\
\hline \multirow[t]{4}{*}{$\begin{array}{l}\text { Oxidation') } \\
\text { (reduced } \mathrm{CO}) *\end{array}$} & $\mathrm{COOH}, \mathrm{OH}, \mathrm{R}-\mathrm{O}-\mathrm{R}$ & $\begin{array}{l}I_{D} / I_{G}=0.91^{a)} \\
(\text { for } G O)\end{array}$ & $\mathrm{I}_{\mathrm{D}} / \mathrm{I}_{\mathrm{G}}=1.10^{\mathrm{a})}$ (hydrazine) & - & 0.22 & {$[87]_{\mathrm{rCO}}$} \\
\hline & & & $\mathrm{I}_{\mathrm{D}} / \mathrm{I}_{\mathrm{G}}=1.38^{\mathrm{a})}\left(\mathrm{H}_{2}\right)$ & & 4.05 & \\
\hline & & & $\mathrm{I}_{\mathrm{D}} / \mathrm{I}_{\mathrm{G}}=1.53^{\mathrm{a})}(\mathrm{EtOH})$ & & 29.1 & \\
\hline & $\mathrm{COOH}, \mathrm{OH}, \mathrm{R}-\mathrm{O}-\mathrm{R}$ & - & $\mathrm{wt} \% \mathrm{O}=5.6\left(\mathrm{Na} / \mathrm{NH}_{3}\right)$ & - & 123 & {$[88]_{\mathrm{rGO}}$} \\
\hline \multirow{6}{*}{$\begin{array}{l}\text { Graftingl) } \\
\text { (diazonium salt) }\end{array}$} & p-bromophenyl & - & $\left.\mathrm{t}=30 \mathrm{~min}^{\mathrm{c}}\right)$ & $\approx 2750$ & $\approx 2800$ & ${ }^{[89]}$ Exfoliation \\
\hline & & & $\left.\mathrm{t}=60 \mathrm{~min}^{\mathrm{c}}\right)$ & & $\approx 2400$ & \\
\hline & & & $\left.\mathrm{t}=90 \mathrm{~min}^{\mathrm{c}}\right)$ & & $\approx 1900$ & \\
\hline & & & $\mathrm{t}=120 \mathrm{~min}^{\mathrm{c})}$ & & $\approx 850$ & \\
\hline & p-nitrophenyl & $\mathrm{I}_{\mathrm{D}} / \mathrm{I}_{\mathrm{G}} \approx 0^{\mathrm{a})}$ & $\mathrm{I}_{\mathrm{D}} / \mathrm{I}_{\mathrm{G}} \approx 1.5^{\mathrm{a})}$ & $\approx 2000$ (on $\mathrm{SiO}_{2}$ ) & $\approx 50$ & ${ }^{[90]}$ Exfoliation \\
\hline & & & & $\approx 15000$ (suspended) & $\approx 200$ & \\
\hline \multirow[t]{3}{*}{$\begin{array}{l}\text { Organo(metallic) molecule }{ }^{11)} \\
\text { ( } \pi-\pi \text { or hydrophobic) }\end{array}$} & $\begin{array}{c}\text { TPA } \\
\text { (Aromatic molecules) }\end{array}$ & $\mathrm{I}_{\mathrm{D}} / \mathrm{I}_{\mathrm{C}} \approx 0^{\mathrm{a})}$ & $\mathrm{I}_{\mathrm{D}} / \mathrm{I}_{\mathrm{G}} \approx 0.4^{\mathrm{a})}$ & . & No obvious change & ${ }^{\left[{ }^{91]} \text { Exfoliation }\right.}$ \\
\hline & Pt-porphyrin & - & - & $\approx 8000(4.2 \mathrm{~K})$ & $\approx 10000(4.2 \mathrm{~K})$ & ${ }^{\left[{ }^{[9]} \text { Exfoliation }\right.}$ \\
\hline & Vanadyl phthalocyanine & - & $\mathrm{n}^{\mathrm{imp}}=5 \times 10^{13} \mathrm{~cm}^{-2 \mathrm{e})}$ & $2000-3000$ & $1500-2300$ & ${ }^{[95]}$ Exfoliation (bilayer) \\
\hline \multirow{7}{*}{$\begin{array}{l}\text { DNA and protein }{ }^{\text {II) }}(\pi-\pi \text { or } \\
\text { hydrophobic) }\end{array}$} & Adenine (A) & - & $0.8 \mathrm{ML}^{\mathrm{d})}$ & $\approx 1620$ & $\approx 1650$ & ${ }^{[97]} \mathrm{CVD}$ \\
\hline & Thymine $(\mathrm{T})$ & & $0.85 \mathrm{ML}^{\mathrm{d})}$ & $\approx 1540$ & $\approx 1700$ & \\
\hline & Cytosine (C) & & $1.1 \mathrm{ML}^{\mathrm{d})}$ & $\approx 1340$ & $\approx 1230$ & \\
\hline & Guanine $(G)$ & & $1 \mathrm{ML}^{\mathrm{d})}$ & $\approx 1640$ & $\approx 1180$ & \\
\hline & ssDNA I (12-mer) & - & - & 305.2 & 237.0 & ${ }^{[101]} \mathrm{CVD}$ \\
\hline & ssDNA II (12-mer) & - & - & 607.1 & 695.2 & \\
\hline & ssDNA (21, 24-mer) & - & - & 2600 & 1600 & ${ }^{[29]}$ Exfoliation \\
\hline $\begin{array}{l}\text { Polyelectrolyte multilayer"I) } \\
\text { (Electrostatic) }\end{array}$ & $\begin{array}{c}\text { polyelectrolyte }\left(\mathrm{PAH}^{+}\right. \\
\text {and PSS) }\end{array}$ & $\mathrm{I}_{\mathrm{D}} / \mathrm{I}_{\mathrm{G}} \approx 0.1^{\mathrm{a})}$ & - & $\approx 1556$ & No obvious change & ${ }^{[96]} \mathrm{CVD}$ \\
\hline \multirow[t]{3}{*}{$\begin{array}{l}\text { Nanoparticle } \\
\text { (van der Waals) }\end{array}$} & Pd nanoparticles & $\mathrm{I}_{\mathrm{D}} / \mathrm{I}_{\mathrm{G}} \approx 0.1^{\mathrm{a})}$ & $\mathrm{I}_{\mathrm{D}} / \mathrm{I}_{\mathrm{C}} \approx 0.1^{\mathrm{a})}$ & 2405 & $\begin{aligned} \approx & 2250(\mathrm{Pd}), 3840 \\
& (\text { Pd-hydrogen })\end{aligned}$ & ${ }^{[106]}$ CVD (bilayer) \\
\hline & Ag nanoparticles & - & $\left.\mathrm{n}^{\mathrm{imp}}=6.2 \times 10^{12} \mathrm{~cm}^{-2 \mathrm{e}}\right)$ & $\approx 810$ & $\approx 810$ & ${ }^{[107]} \mathrm{CVD}$ \\
\hline & & & $\mathrm{n}^{\mathrm{imp}}=9.4 \times 10^{12} \mathrm{~cm}^{-2 \mathrm{e})}$ & & $\approx 600$ & \\
\hline \multirow{3}{*}{$\begin{array}{l}\text { 2D heterostructure }{ }^{I I)} \\
\text { (van der Waals) }\end{array}$} & h-BN & - & Sandwiched & $\approx 15000$ (on $\mathrm{SiO}_{2}$ ) & $\approx 100000$ & ${ }^{[51]}$ Exfoliation \\
\hline & h-BN & - & Sandwiched & - & 197600 & [52]Exfoliation \\
\hline & $h-B N$ & - & Sandwiched & - & $\approx 350000$ & ${ }^{[61]} \mathrm{CVD}$ \\
\hline
\end{tabular}

")covalent functionalization; "Inon-covalent functionalization; *) $\mathrm{GO}$ is used as the starting material for $\mathrm{rCO}$. GO is an insulator; hence no pristine device mobility is provided; a) $I_{D} / I_{G}$ as a measure of $\mathrm{sp}^{2} / \mathrm{sp}^{3}$ in the graphene lattice. Increased ratio correlates to increased $\mathrm{sp}^{3}$ over $\mathrm{sp}^{2}$ (more defects); b) $\mathrm{F} / \mathrm{C}$ as as measure of degree of fluorination: ratio of fluorine over carbon atoms in the material; ${ }^{c} \mathrm{t}=$ reaction time for the functionalization of graphene with diazonium salt; ${ }^{\mathrm{d})} \mathrm{ML}=\mathrm{monolayer}$ of the introduced functional moiety on graphene; ${ }^{\mathrm{e}} \mathrm{n}^{\mathrm{imp}}$ is the amount of functional groups or nanoparticles at the surface of graphene per square centimeter. N.B.: single layer graphene or $\mathrm{rCO}$ unless stated otherwise; $\mathrm{T}=293 \mathrm{~K}$ unless stated otherwise. 


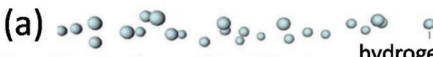
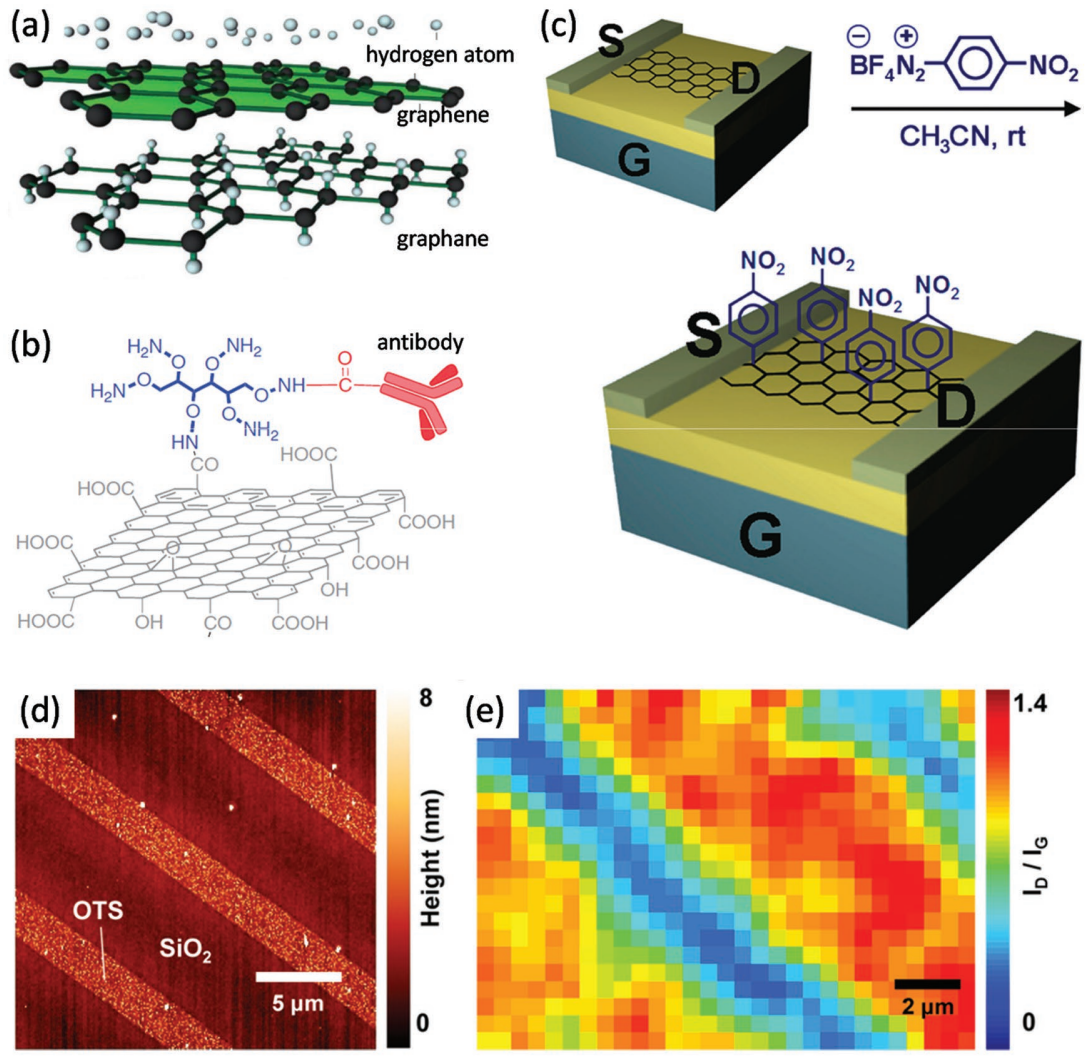

(f)

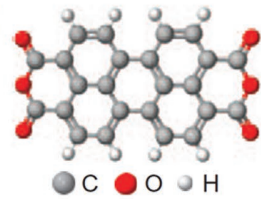

(g)
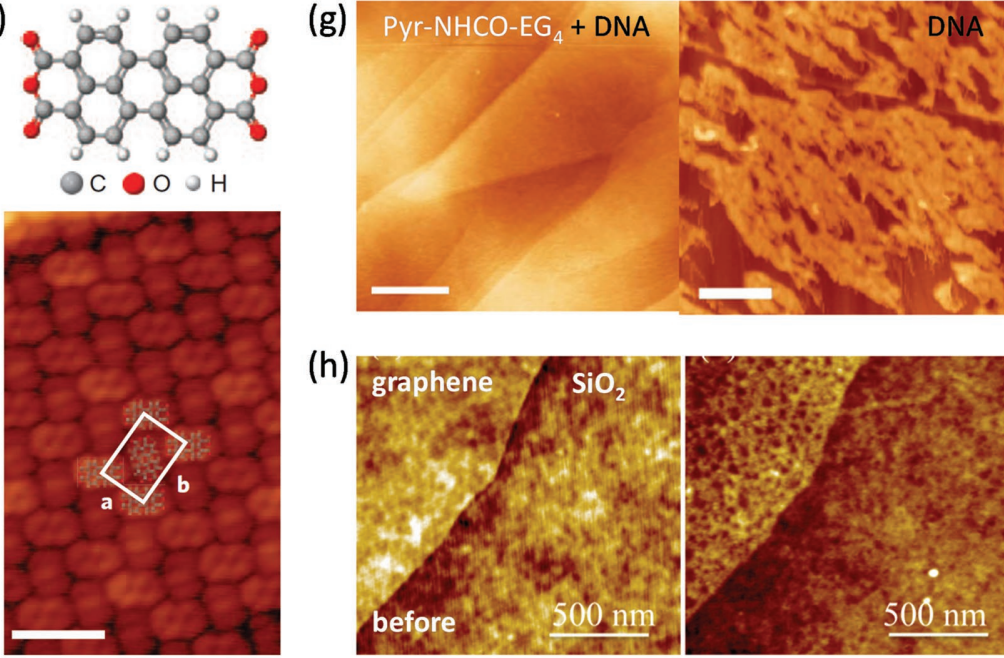

(h)
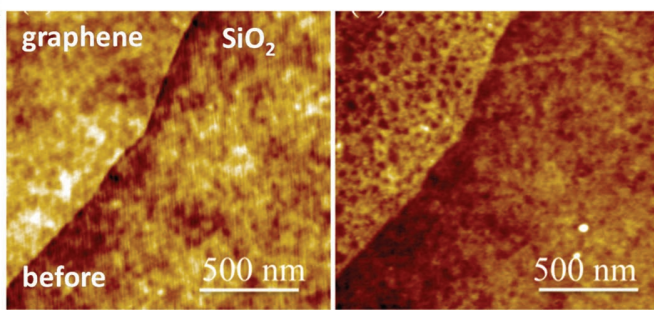

Figure 3. Surface chemical functionalizations of graphene materials. a) Graphene layer (in green) is attacked by cold plasma hydrogen atoms to produce graphane. Reproduced with permission. ${ }^{[23]}$ Copyright 2009, the American Association for the Advancement of Science. b) Bioconjugation of PEGylated GO with antibody. Reproduced with permission. ${ }^{[26]}$ Copyright 2013, Nature Publishing Group. c) Schematic of the chemical functionalization of a GFET device with 4-nitrobenzene diazonium tetrafluoroborate (4-NBD). Reproduced with permission. ${ }^{[126]}$ Copyright 2010, American Chemical Society. d) AFM image of octadecyltrichlorosilane (OTS) lines patterned on $\mathrm{SiO}_{2}$ surface. e) Raman mapping of $\mathrm{I}_{\mathrm{D}} / \mathrm{I}_{\mathrm{G}}$ for graphene after 4-NBD reactions: $10 \mathrm{mM} 4-\mathrm{NBD}$ in aqueous solution with $0.5 \mathrm{wt} \%$ sodium dodecyl sulfate (SDS) at $35^{\circ} \mathrm{C}$ for 1.5 h. Reproduced with permission. ${ }^{[123]}$ Copyright 2012, Nature Publishing Group. f) STM image of a self-assembled monolayer of an aromatic molecule (perylene-3,4,9,10-tetracarboxylic-3,4,9,10-dianhydride, PTCDA) (gas-phase deposition) on a graphene surface (scale bar is $3 \mathrm{~nm}$ ). Upper panel: molecular structure of PTCDA. Reproduced with permission. ${ }^{\text {92] }}$ Copyright 2009, Nature Publishing Group. g) Left panel: AFM of highly oriented pyrolitic graphite (HOPG) covalent approaches reliably modify the graphene surface with various functional biochemical molecules ${ }^{[26]}$ by reacting with the $\mathrm{sp}^{2}$ carbon centers in the aromatic lattice, introducing $\mathrm{sp}^{3}$ centers at the reaction sites. Precautions have to be taken as such chemical modification reduces the flatness, but more importantly, destroys the aromaticity of the graphene lattice and renders the modified material inferior in terms of electrical mobility compared to pristine graphene (and noise performances as well, but not shown in Table 2). On the contrary, non-covalent approaches provide the opportunity to functionalize graphene without disrupting its intrinsic aromaticity. ${ }^{[32]}$ Instead, an increase in the mobility of functionalized devices compared to pristine devices was observed from time to time, especially for h-BN sandwiched graphene samples (see Table 2). Thus, non-covalent strategies are very appealing for realizing high-performance sensors.

\subsection{Covalent Functionalizations}

Covalent chemical modification of graphene allows engineering the properties of graphene to a large extend, particularly with the scope of band gap engineering, surface modification, and biointerfacing. ${ }^{[109]}$ Introducing atomic hydrogen or fluorine into the honeycomb scaffold, reveals the possibility to continuously transform this highly conductive zero-band gap semimetal into an insulator known as graphane ${ }^{[23]}$ (Figure 3a) or 2D Teflon, ${ }^{[24,81]}$ as initially proposed by the Manchester group. Regarding sensing applications, calculations showed that (partially) hydrogenated graphene has a high affinity for $\mathrm{NO}_{2},{ }^{[111]}$ while graphane doped with $\mathrm{Li}$ adatoms was predicted to be sensitive to $\mathrm{H}_{2} \mathrm{~S}$ and $\mathrm{NH}_{3}{ }^{\left[{ }^{[12]}\right]}$ Moreover, the reduced carrier mobility of highly hydrogenated graphene is still sufficient for sensor applications. ${ }^{[113]}$ Fluorographene, on the other hand, was applied for the detection of ammonia, ${ }^{[114]}$

incubated for 5 min with a solution of $3 \mathrm{M} \mathrm{KCl}$ and $8 \mathrm{M}$ urea and rinsed with ultrapure water (scale bar is $200 \mathrm{~nm}$ ). Right panel: HOPG incubated for $5 \mathrm{~min}$ with single-stranded M13 DNA $\left(10 \mathrm{ng} \mathrm{Il}^{-1}\right)$ in the same buffer (scale bar is $200 \mathrm{~nm}$ ). Reproduced with permission. ${ }^{[128]}$ Copyright 2013, Nature Publishing Group. h) AFM topographic image of graphene before (left panel) and after (right panel) incubation with the peptide. Reproduced with permission. ${ }^{[31]}$ Copyright 2012, American Chemical Society. 
ascorbic acid, and uric acid. ${ }^{[15]}$ The fluorine-enriched material could also be further functionalized with thiol groups for genosensing. ${ }^{[16]}$ Underlying mechanisms and selectivity of the sensor are still under debate.

Separately, graphene sheets are now routinely covalently modified with oxygen functional groups (e.g., carboxyl, hydroxyl and epoxy moieties, see also Figure $3 \mathrm{~b}$ ) by using oxidative reactions, forming graphene oxide (GO), a material known since the early $1960 \mathrm{~s} .{ }^{[117]}$ The synthetic process consists in dispersing graphite into stable single layer GO and is suitable for large scale production of dispersible single layer graphene using a thermal or chemical reduction step. The resulting material is often referred to as reduced GO, or rGO. ${ }^{[118]}$ Remarkably, when used as an active sensing electrode, GO and rGO usually show improved sensing responses, presumably due to the large concentration of defects compared to near defect-free single layer graphene obtained via mechanical exfoliation of graphite. ${ }^{[25,119]}$ One of the first works on rGO as an active material for highperformance molecular sensing describes a conductance change of the rGO networks upon exposure to trace levels of vapor (including three main classes of chemical-warfare agents and an explosive at parts-per-billion concentrations). ${ }^{[25]}$ It was shown that the optimal defect density should balance the gains in the sensor response against the rapid degradation in low frequency $1 / f$ noise due to the increased density of defects. ${ }^{[25]}$ The difficulties in controlling the density of the defect as well as the lack of knowledge on the nature of the defect, however, represents significant limitations for utilizing GO or rGO for sensing applications. Reactive oxygen-rich groups, inherently present on $\mathrm{rGO}$, can be exploited to synthetically conjugate the material with various chemical or biological groups. ${ }^{[26]} \mathrm{A}$ viable synthetic strategy is depicted in Figure 3b: a GO-polyethylene glycol dispersion (i.e., PEGylated GO) was prepared; the hydrophilic six-armed PEG- $\mathrm{NH}_{2}$ could then be labelled by conjugating an antibody (for potential antibody-antigen detection ${ }^{[26]}$ ).

Hydrogenated graphene, fluorinated graphene (or halogenated graphene ${ }^{[120]}$ in general), and GO (or rGO) are the few examples of materials that resulted from covalent modification of the graphene scaffold. Instead of providing an extensive list of the methods available to induce such modifications, we will continue with discussing a grafting strategy, frequently applied to covalently attach chemical moieties to graphene surface (or edges) via free-radical reactions. [27,28,109,121-125] Graphene grafting uses alkyl or aryl diazonium salts as grafting agents, where the diazonium salt precursor is first chemically or electrochemically reduced (liberating nitrogen gas), to form a reactive alkyl or aryl radical that reacts with the aromatic system of the graphene sheet (the conductive channel of the transistor device fabricated on a $200 \mathrm{~nm} \mathrm{SiO} /$ /highly doped Si substrate as shown in Figure 3c). ${ }^{[126]}$ The disruption of the aromatic system by transforming the hybridization of carbon atoms from $\mathrm{sp}^{2}$ to $\mathrm{sp}^{3}$ results in a remarkable decrease in graphene conductivity, which can be controlled by reaction time (see also Table 2). The reaction efficiency depends on several parameters: the number of graphene layers, ${ }^{[122]}$ the electrostatic environment, ${ }^{[123]}$ and the defect density on the graphene surface. ${ }^{[124]}$ A previous study exploited the graphene reactivity, induced by electrostatic charge doping on different substrates using reactivity imprint lithography (RIL). ${ }^{[123]}$ The RIL technique made use of a polydimethylsiloxane (PDMS) stamp to pattern octadecyltrichlorosilane (OTS) lines on a $\mathrm{SiO}_{2} / \mathrm{Si}$ substrate (Figure 3d). During the electrografting of graphene with 4-nitrobenzene diazonium tetrafluoroborate (4-NBD), ${ }^{[28]}$ bare $\mathrm{SiO}_{2}$-supported graphene showed a stronger reactivity with the diazonium salt than graphene resting on OTS-protected $\mathrm{SiO}_{2}$ (Figure 3e). OTS increases the distance between the graphene sheet and the charged impurities in the $\mathrm{SiO}_{2}$ substrate, rendering the portion of graphene resting on it less reactive to the 4-NBD. ${ }^{[123]}$ Similarly, in case of GO (or rGO), grafting chemistries are best represented by localized reactivity of the carboxyl, carbonyl, and other oxygen-containing groups by substitution reactions. ${ }^{[124]}$

\subsection{Non-covalent Functionalization}

As mentioned in the previous section, non-covalent functionalization has the major advantage of fully preserving the graphene lattice (i.e., the aromaticity), and thus the electrical performances (see Table 2). In addition, non-covalent bond can also be quite strong. For example, the $\pi-\pi$ interactions of graphene-benzene and naphthalene result in a considerable binding energy of almost $0.1 \mathrm{eV}$ per carbon atom; consistently, the binding energy of graphene-TTP (tetraphenylporphyrin) was calculated to be $3.2 \mathrm{eV}$, i.e., $\approx 90 \%$ of a typical C-C covalent binding energy $(\approx 3.6 \mathrm{eV}) .{ }^{[127]}$ Given the aforementioned advantages, it is a common approach to anchor a biomolecule onto the graphene surface using an aromatic linker group via noncovalent bonds with excellent sensing performance in aqueous solutions. ${ }^{[109]}$ Still, we would like to note here that non-covalent functionalization is expected to be less compatible with long term usage, at least if compared to stronger covalent functionalization (although the covalent modifications of graphene inevitably lead to a severe degradation in the electrical properties). Nevertheless, non-covalent functionalization could also be an asset if the sensor surface has to be regenerated, for example, for recycling the sensor devices.

In general, non-covalent graphene functionalization approaches can be classified based on their corresponding intermolecular interactions, including $\pi-\pi$ or hydrophobic stacking, electrostatic interaction, and van der Waals interaction as also shown in Table $1 .^{[109]}$ The self-assembly process of these molecules on the surface of graphene could be highly controlled and accurately characterized in favor of an actual sensor design. ${ }^{[109,110]}$ For example, Figure $3 \mathrm{f}$ shows a scanning tunneling microscopy (STM) image of well-ordered aromatic perylene-3,4,9,10-tetracarboxylic-3,4,9,10-dianhydride (PTCDA) molecules on graphene (as indicated by the $\mathbf{a}$ and $\mathbf{b}$ vectors), where $\pi-\pi$ interaction are the driving force of the self-assembly. ${ }^{[92]}$ The perylene-based monolayer is stable and robust even when exposed to ambient conditions. $\pi-\pi$ or hydrophobic interactions between aromatic surface and nucleic acid moieties can also facilitate the decoration of graphene surface with single-stranded DNA (ssDNA) as shown in Figure $3 \mathrm{~g}$ (right panel, highly oriented pyrolytic graphite (HOPG) was applied in this case). ${ }^{[128]}$ This strong nonspecific ssDNA adsorption can be avoided by first self-assembling a monolayer of pyrene ethylene glycol, thus rendering the surface of graphene hydrophilic and preventing ssDNA adsorption via hydrophobic interactions (left panel, Figure 3g). Besides DNA, proteins ${ }^{[102]}$ or peptides ${ }^{[30,31]}$ containing aromatic moieties 
could also self-assemble on a graphene scaffold. As illustrated in Figure $3 \mathrm{~h}$, the incubation of graphene with the peptides resulted in the formation of an uniform mesh-like layer whilst silicon oxide surface was unaffected. This indicates that the adsorption occurred specifically on graphene. ${ }^{[31]}$

Electrostatic interaction is another driving force of the non-covalent assembly. For instance, voltage-biased graphene can act as an electrophoretic electrode for immobilization of charged biomolecules. The subsequent detection of complementary analysts can be achieved by using the same graphene transistor devices. ${ }^{[22,96,129]}$

As suggested by Geim and co-workers, ${ }^{[108]}$ weak van der Waals-like interaction between layers could be exploited to sandwich (a process called "encapsulation") graphene with other 2D layers of, e.g., $\mathrm{MoS}_{2}$, mica, or hexagonal boron nitride (h-BN). This innovative technique allows the formation of unprecedented multilayer heterostructures that may be used in devices with adjustable and astonishing electronic properties. For example, by encapsulating graphene in a h-BN stacking layer, researchers managed to obtain very high electric performances GFETs, including an exceptionally high carrier mobility of $140000 \mathrm{~cm}^{2} \mathrm{~V}^{-1} \mathrm{~s}^{-1}$ at room temperature, which is close to the theoretical limit as imposed by acoustic phonon scattering. This extremely high mobility could be ascribed to very clean interfaces above and below graphene and effective screening of all the defects. ${ }^{[17]}$ Very recently, even higher mobilities, up to a staggering $197600 \mathrm{~cm}^{2} \mathrm{~V}^{-1} \mathrm{~s}^{-1[52]}$ and $350000 \mathrm{~cm}^{2} \mathrm{~V}^{-1} \mathrm{~s}^{-1}$, [61] have been observed for hBN-sandwiched graphene samples. One could also explore various 2D crystals as active sensing elements, $\mathrm{MoS}_{2}$ or h-BN capped $\mathrm{MoS}_{2},{ }^{[53,130]}$ for instance. Please note that even in a stack such as encapsulated graphene, the encapsulating layers can be functionalized in the quest of sensing (with the requirement that the encapsulating layer is sufficiently thin).

As previously discussed, chemical functionalization is essential for unlocking the sensing potential of graphene surface, but important is also to realize that chemical functionalization also plays a critical role in passivating the surface of graphene. Surface passivation against unwanted non-specific binding (pyrene ethylene glycol to prevent any hydrophobic interactions, ${ }^{[128]}$ for example) is crucial to achieve very low detection limits in the presence of high ionic background levels and to avoid false positives when complex biological samples are assayed. [131]

Importantly, the transfer of large and clean (and crack- and fold-free) graphene sheets is still a critical challenge. Long chain polymers including poly(methyl methacrylate) (PMMA) - conventionally used for transferring two-dimensional materials - irreversibly adsorb on the graphene surface, yielding a range of contaminations with unwanted chemical functions. ${ }^{\text {[132] }}$ It is therefore a necessity to take into account the influences of these possible polymer residues as they impede the functionalization of the graphene surface (which is actually not always discussed, nor clarified in the literature). There is therefore also a large demand for decent polymer-free transfer methods. ${ }^{[133]}$

\subsection{Graphene Lipid Superstructures: Towards Graphene Bioelectronics}

Graphene bioelectronics represents a highly interdisciplinary field that combines material science with biology and electronics at the interface. The rapid expansion in this field offers the great potential to construct innovative biological cellular sensor devices to overcome existing challenges in bioelectronics and therefore opens up new opportunities in fundamental biology and healthcare. ${ }^{[68,134]}$ These challenges include the shrinking of the electronic dimensions to microor even nanoscale and large-scale integration for high-resolution sampling, ${ }^{[135]}$ but particularly, a complex but well-defined biocompatible interface between graphene surface and cell is at the core of graphene bioelectronics.

Lipids, as major constituents of the cell membrane, provide a physical barrier between the interior and the exterior of a cell. Along with their associated proteins, lipids are responsible for the key functions of a cell such as the highly controlled selectivity of passage of molecules and ions. ${ }^{[136]}$ Despite the hitherto limited knowledge on lipid-graphene interaction, these basic understandings are actually of vital importance as the starting point of graphene biointerfacing. Molecular dynamics simulations of the interaction between pristine graphene and lipid bilayers revealed a well-defined graphene-sandwiched superstructure most presumably achieved by hydrophobic interactions (Figure 4a). ${ }^{[137]}$ Precise patterning of phospholipid molecules directly on exfoliated graphene (the left panel of Figure 4b) can also be achieved by using dip-pen nanolithography. ${ }^{[138]}$ The graphene surface favors a merged and uniformed lipid layer in comparison to the lipid patches patterned on silicon oxide in the same conditions, as the lipids have tendency to slip and spread on the graphene surface (the right panel of Figure 4b).

Lipid interaction with $\mathrm{GO}^{[139]}$ can be harvested to control the assembly of GO sheets into large superstructures as well as to unravel the potential toxicity of graphene derivatives to cells. ${ }^{[140]}$ Previous studies in a Langmuir-Blodgett trough revealed that the negatively charged GO sheets dispersed in water interact with the positively charged lipids head groups present at the air/water interface mainly with two configurations: i) GO sheets positioned vertically to the interface ${ }^{[141]}$ or ii) GO sheets parallel to the interface. ${ }^{[142]}$ To understand how lipids interact with GO on solid substrates, GO has been incubated in the presence of various lipid compositions. ${ }^{[143,144]}$ Figure 4c shows a lipid membrane that is first formed on a $\mathrm{SiO}_{2}$ substrate by vesicle fusion assembly. Second, the negatively charged GO specifically adsorbs on the positively charged lipids, and induces rupture of further adsorbed liposomes, resulting in well-organized lipid-GO multilayered structures. ${ }^{[143]}$

Advantageously, unlike the conventional solid electronics with rigid surfaces, graphene electronics are well-known for the fabrication of flexible and transparent electrodes. ${ }^{[145]}$ Therefore graphene provides a flexible and conducting substrate that interfaces well with the soft, 3D biological systems. ${ }^{[146-148]}$ For example, the mechanical flexibility and electrical functions of graphene membrane can be used to achieve a strongly coupled electromechanical biointerface by coating yeast cells with an ultrathin layer of rGO. ${ }^{147]}$ Nevertheless, most researches on graphene biointerfaces still use graphene on rigid solid substrates at an intermediate stage mainly focusing on understanding the complicated sensing mechanisms (as the reconfiguration of the fluidic-like lipid layer has to be considered). ${ }^{[149-151]}$ For example, 
(a)

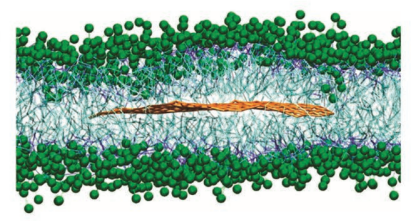

(b)
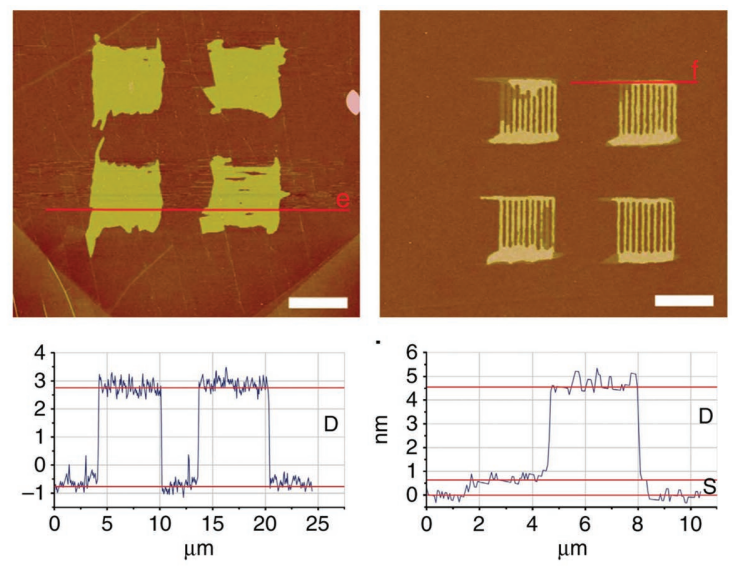

(c)

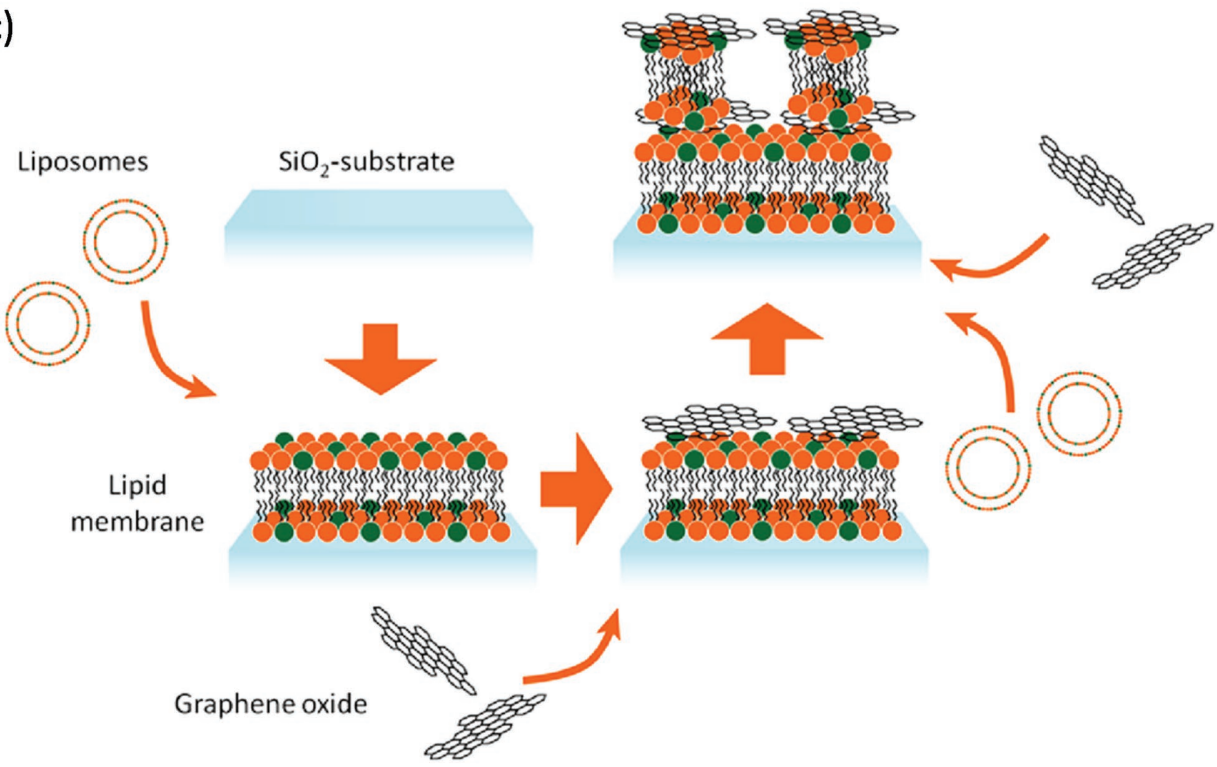

Figure 4. Non-covalent functionalizations of graphene with lipids. a) Stable superstructure of graphene sheet sandwiched within the hydrophobic core of a phospholipid bilayer membrane. Reproduced with permission. ${ }^{[137]}$ Copyright 2012, American Chemical Society. b) AFM images (scale bar is $5 \mu \mathrm{m}$ ) and the respective height profiles of phospholipid patches on graphene and on silicon oxide, respectively. Graphene induces a merged and uniform lipid patch compared to silicon oxide in the same conditions. Reproduced with permission. ${ }^{[138]}$ Copyright 2013, Nature Publishing Group. c) Schematic representation of $\mathrm{GO}$ sheets interacting with lipid forming stacked and multilayer structures on $\mathrm{SiO}_{2}$ substrate (by vesicle fusion assembly). Reproduced with permission. ${ }^{[143]}$ Copyright 2012, American Chemical Society.

a gram-negative bacteria biomimetic membrane was deposited on CVD GFETs (fabricated on a $\mathrm{SiO}_{2} / \mathrm{Si}$ substrate) for detecting magainin 2, an antimicrobial agent. The presence of magainin 2 disrupts and thins the lipid membrane from a thickness of $\approx 5 \mathrm{~nm}$ to $\approx 3 \mathrm{~nm} .{ }^{[150]}$ This change in membrane thickness and integrity lead to a significant change in the liquid gate coupling, and thus to a noticeable field effect which could be measured by the GFETs. The self-assembly processes of charged lipid bilayers can also induce a sensing signal in the GFET due to surface charge aggregation. Such GFETs interfaced with biomimetic membrane can even provide enough sensitivity to investigate individual ion channel activity during the insertion of a pore-forming membrane protein. ${ }^{[151]}$ Graphene bioelectronics for cellular sensors will be further discussed in the next Section 4.3.

\section{Current Trends \& Efforts in Biochemical Sensing at the Surface of GFETs}

There has been a vast interest of industry, society, and scientific community in applying graphene materials for biochemical sensing applications, for portable point-of-care devices for remote diagnostics, for environmental monitoring, and even for DNA sequencing technologies, etc. ${ }^{[65-67]}$ The outcomes of researches in this area, however, did not reach the market yet, ${ }^{[152]}$ although ultimate single molecule sensitivity has been demonstrated and prototype biosensor chips in various forms have also been developed. ${ }^{[65-67]}$ In the following sections, we will critically review the current trends in the development of GFET-based gas and ion sensors, protein and DNA sensors, and cellular sensors in revisiting the 
Table 3. Sensing performances of GFETs for a selected list of frequently reported analytes.

\begin{tabular}{|c|c|c|c|c|c|}
\hline Analyte & & $\begin{array}{l}\text { Graphene device mobility } \\
\qquad\left[\mathrm{cm}^{2} \mathrm{~V}^{-1} \mathrm{~s}^{-1}\right]\end{array}$ & Detection limit & $\begin{array}{l}\text { Corresponding sensing response } \\
|\Delta \mathrm{I} / \mathrm{I} \times 100 \%| \text { or }|\Delta \mathrm{R} / \mathrm{R} \times 100 \%|\end{array}$ & Refs (comments) \\
\hline \multirow[t]{7}{*}{ Gas } & $\mathrm{NO}_{2}$ & - & $1 \mathrm{ppm}$ & $0.99 \%$ & {$[155] \mathrm{rGO}$} \\
\hline & & $\approx 10$ & $20 \mathrm{ppm}$ & $21 \%$ & ${ }^{[156]} \mathrm{CVD}$ \\
\hline & & $\approx 5000$ & $1 \mathrm{ppm}$ & $\approx 4 \%$ (noise level: $\approx 0.1 \%$ ) & ${ }^{[157]}$ Exfoliation \\
\hline & $\mathrm{NH}_{3}$ & - & $200 \mathrm{ppm}$ & $10.2 \%$ & ${ }^{[155]} \mathrm{r} \mathrm{CO}$ \\
\hline & & $\approx 10$ & $550 \mathrm{ppm}$ & $10 \%$ & [156]CVD \\
\hline & & $\approx 5000$ & $1 \mathrm{ppm}$ & $\approx 4 \%$ (noise level: $\approx 0.1 \%$ ) & ${ }^{[157]}$ Exfoliation \\
\hline & & 5500 & $10^{3} \mathrm{ppm}$ & $1.5 \%$ & $\begin{array}{l}{ }^{[158]} \text { Exfoliation, } \\
\text { annealed }\end{array}$ \\
\hline \multirow[t]{13}{*}{ Ion } & $\mathrm{H}^{+}$ & - & $0.67 \mathrm{pH}$ & $27.8 \%$ & ${ }^{[159]} \mathrm{rCO}$ \\
\hline & & - & $0.34 \mathrm{pH}$ & $18 \%$ & ${ }^{[160]} \mathrm{CVD}$ \\
\hline & & 3600 & - & $99 \mathrm{mV} /$ decade $^{\mathrm{a})}$ & ${ }^{[161]}$ Exfoliation \\
\hline & & 4400 & $0.21 \mathrm{pH}$ & $8.7 \%$ & ${ }^{[162]}$ Exfoliation \\
\hline & & 5000 & $0.21 \mathrm{pH}$ & $12.8 \%$ & [163]Exfoliation \\
\hline & $\mathrm{K}^{+}$ & $\approx 300$ & $100 \mu \mathrm{M}$ & $40 \mathrm{mV} /$ decade $^{\mathrm{a})}$ & ${ }^{[37]} \mathrm{CVD}$ \\
\hline & & - & $10 \mathrm{nM}$ & $7.8 \mathrm{mV} /$ decade $^{\mathrm{a})}$ & ${ }^{[164]}$ Exfoliation \\
\hline & $\mathrm{Na}^{+}$ & - & $1 \mathrm{nM}$ & $1.5 \mathrm{mV} /$ decade $^{\text {a) }}$ & ${ }^{[165]}$ Exfoliation \\
\hline & $\mathrm{Ca}^{2+}$ & - & $1 \mu \mathrm{M}$ & $\approx 4 \%$ (SNR: $20-30$ ) & {$[166] \mathrm{r} \mathrm{CO}$} \\
\hline & $\mathrm{Cd}^{2+}$ & - & $1 \mathrm{nM}$ & $\approx 1 \%$ (SNR: 15-20) & \\
\hline & $\mathrm{Hg}^{2+}$ & - & $1 \mathrm{nM}$ & $\approx 2 \%$ (SNR: 25-30) & \\
\hline & & $\approx 4000$ & $10 \mathrm{ppm}$ & (back gate voltage shift: $\approx 6.2 \mathrm{~V}$ ) & ${ }^{[167]}$ Exfoliation \\
\hline & $\mathrm{Pb}^{2+}$ & - & $37.5 \mathrm{ng} / \mathrm{L}$ & (liquid gate voltage shift: $\approx 35 \mathrm{mV}$ ) & ${ }^{[168]}$ Exfoliation \\
\hline \multirow[t]{6}{*}{ DNA } & ssDNA (20-mer) & 0.068 & $0.175 \mathrm{mM}$ & $71 \%$ & ${ }^{[99]} \mathrm{GO}$ \\
\hline & ssDNA (33-mer) & - & 48 nM (2.4 nM calc. @ SNR=3) & $0.6 \%$ (SNR: 60) & {$[169] \mathrm{rGO}$} \\
\hline & Fully complementary ssDNA (12-mer) & $\approx 150-700$ & $0.001 \mathrm{nM}$ & $\approx 30 \%$ (in carrier density) & ${ }^{[101]} \mathrm{CVD}$ \\
\hline & & & $0.01 \mathrm{nM}$ & $\approx 12 \%$ (in mobility) & \\
\hline & 1-base mismatched ssDNA (12-mer) & & $0.001 \mathrm{nM}$ & - & \\
\hline & & & $0.01 \mathrm{nM}$ & $0 \%$ (in mobility) & \\
\hline \multirow[t]{3}{*}{ Protein } & Protective antigen (Anthrax toxin) & - & $1.2 \mathrm{aM}$ & 1.5\% (@12 aM) & {$[170] \mathrm{rGO}$} \\
\hline & Bovine serum albumin (BSA) & $\approx 1250-1750$ & $300 \mathrm{pM}$ & $\approx 0.36 \%$ & ${ }^{[162]}$ Exfoliation \\
\hline & Immunoglobulin E (IgE) & - & $290 \mathrm{pM}$ & $\approx 0.3 \%$ & ${ }^{[171]}$ Exfoliation \\
\hline \multirow[t]{4}{*}{ Glucose } & Glucose & - & $1 \mathrm{nM}$ & $64 \%$ & {$[172] \mathrm{rGO}$} \\
\hline & & 2298 & $1.25 \mathrm{mM}$ & $\approx 25 \%$ & ${ }^{[173]} \mathrm{CVD}$ \\
\hline & & - & $30 \mathrm{nM}$ & $\approx 1.1 \%$ & {$\left[{ }^{[174]} \mathrm{CVD}\right.$} \\
\hline & & - & $0.5 \mu \mathrm{M}$ & $\approx 0.5 \%$ & {$\left[{ }^{[75]} \mathrm{CVD}\right.$} \\
\hline \multirow[t]{2}{*}{ Cell } & Embryonic chicken cardiomyocyte cell & 4000 & $\approx 3.5 \mathrm{mV}$ & $(S N R \geq 4)$ & ${ }^{[176]}$ Exfoliation ${ }^{\text {b) }}$ \\
\hline & HL-1 mouse atrial tumor cell & 3000 & $100 \mu \mathrm{V}$ & $(S N R>10)$ & {$[177] C V D$} \\
\hline
\end{tabular}

a) $\mathrm{mV} /$ decade: liquid gate voltage shift in the Dirac point of a GFET per decade (ion concentration); b) in combination with Si-nanowire device.

ambiguous cases and in meeting the social/scientific needs. A brief introduction to the graphene electrochemical sensors will also be given as their operation and sensing mechanism can be regarded to be complementary to GFET sensing technologies..$^{[153,154]}$ Before discussing the separated cases, we summarized the sensing performances (and the electrical properties) of GFETs in Table 3 for a selected lists of frequently reported analytes.
As we pointed out in the previous Sections 2.2 and 2.3, it is preferential to use high mobility graphene for sensing applications as: 1. a higher mobility implies a larger sensing response; 2. a higher mobility complies with less defects and surface contaminations, which is in favor of an improved noise performance. Such trend is evidenced, for example, in case of gas sensors. Table 3 showed that for gas detection of both $\mathrm{NO}_{2}$ and $\mathrm{NH}_{3}$, exfoliated graphene with high mobility 
$\left(\approx 5000 \mathrm{~cm}^{2} \mathrm{~V}^{-1} \mathrm{~s}^{-1}\right)$ generally demonstrated a much better detection limit $\approx 1 \mathrm{ppm}$ compared to $\approx 1-550 \mathrm{ppm}$ of CVD or rGO with lower mobilities. We may also relate the decrease in the detection limit of DNA molecules - from $\approx 0.001-0.01 \mathrm{nM}$ (CVD graphene) to $\approx 48 \mathrm{nM}(\mathrm{rGO})$, and to $\approx 0.175 \mathrm{mM}(\mathrm{GO})-$ to the degradation in the electrical properties of (functionalized) graphene. In our listed cases of DNA detection, we made the comparisons as all the sensing reactions were based on short chain DNA hybridization, and thus sharing comparable (at least to a certain extent) bonding constants. We noted here that, it might not always be fair to make such comparisons, precautions have to be taken as surface condition (functionalization) of graphene as well as the device geometry might be different from sample to sample (and is not always reported in the literature). For example, Table 3 showed that annealing exfoliated graphene exhibited a comparable carrier mobility to as-fabricated exfoliated graphene (from $\approx 5000 \mathrm{~cm}^{2} \mathrm{~V}^{-1} \mathrm{~s}^{-1}$ to $\approx 5500 \mathrm{~cm}^{2} \mathrm{~V}^{-1} \mathrm{~s}^{-1}$ ), but also a strikingly decreased sensitivity to $\mathrm{NH}_{3}$ gas (from $\approx 1 \mathrm{ppm}$ to $\approx 1000 \mathrm{ppm}$ ) due to mainly the difference in their surface conditions (see the following section for detailed discussions).

\subsection{GFET Gas and Ion Sensors}

The very first chemical sensors fabricated using pristine mechanically exfoliated graphene flakes (Figure 5a) have shown very high sensitivity, down even to single molecule detection for the chemisorbed gaseous $\mathrm{NO}_{2}$ (and a detection limit higher than $1 \mathrm{ppm}$ for $\mathrm{NH}_{3}$ as shown in Figure 5b, at room temperature). ${ }^{[5]}$ The unprecedented high sensitivity was explained as a consequence of the extraordinary low intrinsic noise as well as the largest response of the Hall geometry around the CNP. However, this high chemical sensitivity of graphene towards physisorbed gaseous $\mathrm{NH}_{3}$ is a surprise, considering the fact that graphene is intrinsically chemically inert. ${ }^{[12]}$ Indeed, in more recent studies, inert electrical responses (i.e., no response) for gaseous $\mathrm{NH}_{3}$ were observed by annealing the exfoliated graphene at high temperature $(400$ ${ }^{\circ} \mathrm{C}$ ) in $\mathrm{Ar} / \mathrm{H}_{2}$ atmosphere to remove possible polymer contaminations and produce atomically clean graphene sheets. ${ }^{[178]}$ This inert sensing behavior of the cleaned graphene sensors is robust even upon the exposure to $\mathrm{NH}_{3}$ vapor at a concentration of $1000 \mathrm{ppm}$ (Figure 5c) ${ }^{[11]}$ and to dimethylmethylphosphonate (DMMP) vapor at a concentration of $100 \mathrm{ppm}$ (Figure 5d). ${ }^{[29]}$ It is now widely accepted that clean graphene should be inert to the presence of most of the gas molecules, although it is possible to amplify the more subtle dipole moment of a charge neutral gas molecule by switching it and mixing the modulated dipole signal in a high frequency setup for detection. ${ }^{[179]}$ The previous observed sensitive responses of pristine graphene to gas molecules could be, therefore, ascribed to the sensitivities of defects or polymer contaminations introduced during device fabrication. The edge of graphene, also plays a crucial role in the determination of its physical, electronic and chemical properties and thus in the sensing properties. As an example, holey rGO could be fabricated by using enzymatic oxidation followed by reduction with hydrazine. ${ }^{[180]}$ Such defective graphene - with abundant edge defects - exhibited a large and selective electronic response toward the detection of hydrogen, particularly when decorated with Pt nanoparticles. ${ }^{[180]}$ In principle, the substrate surface conditions are also suspected to influence the edge of graphene, and hence the sensing properties of the GFET devices especially at nanoscale. ${ }^{[181]}$

GFET based $\mathrm{pH}$ sensors measure the protonation and deprotonation at the (functionalized) graphene surface. They hold great potentials especially in food industry and medical applications for glass-free $\mathrm{pH}$ measurement requiring the highest level performance, small size, and/or flexibility. ${ }^{[182]}$ In earlier reports, GFET based $\mathrm{pH}$ sensors exhibited large variation in their $\mathrm{pH}$ sensitivities, ranging from a low value of $12 \mathrm{mV} / \mathrm{pH}$ to a value of $99 \mathrm{mV} / \mathrm{pH} .{ }^{[7-10,21]}$ The latter value is even larger than the thermodynamically allowed maximal shift of $60 \mathrm{mV} / \mathrm{pH}$ (the so-called Nernst value) at room temperature. ${ }^{[8]}$ It became clear later on that the large range of $\mathrm{pH}$-induced gate shifts observed in the previous literature could be ascribed to defects as well as surface contaminations reflecting the quality of as-fabricated graphene. ${ }^{[66,183]}$ Practically GFETs are normally composed of CVD graphene and contain therefore defects in the lattice introduced during growth. ${ }^{[55]}$ Exfoliated samples are also subject to various polymer and metal contaminations coming from the fabrication process. ${ }^{[11]}$ These defects and contaminations also account for the discrepancies reported in earlier literature on pristine graphene chemical sensors. ${ }^{[5-7]}$ Such defect-induced ionic response can be suppressed by passivating the graphene layer with inert aromatic molecules such as fluorobenzene. ${ }^{[12]}$ As a consequence of its ideal hydrophobic surface with a very small amount (ideally zero) of dangling bonds, such a clean GFET should be inert to the change of electrolyte compositions, and could therefore act as a novel solid-state reference electrode that senses only the electrostatic potential in aqueous electrolytes unless a chemo-adsorption or a physicoadsorption of charged ions is considered. ${ }^{[184]}$ On the contrary, by functionalizing the GFETs with active groups, for example, with proton sensitive phenol or $\mathrm{K}^{+}$sensitive crow ethers conjugated with aromatic molecular anchor groups, a $\mathrm{pH}$ response up to $\approx 49 \mathrm{mV} /$ decade or a $\mathrm{K}^{+}$ionic response up

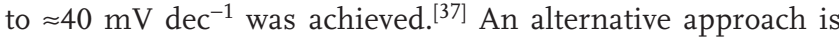
to directly coat an ion-selective membrane (ISM) on graphene surface. A recent report has demonstrated the selective detection of $\mathrm{K}^{+}$ions $\left(-8 \mathrm{mV} \mathrm{dec}{ }^{-1}\right)$ over $\mathrm{Na}^{+}$ions $\left(-0.2 \mathrm{mV} \mathrm{dec}^{-1}\right)$ in an electolyte solution with an ISM composed of valinomycin, a $\mathrm{K}^{+}$selective ionophore. ${ }^{[185]}$

Heavy metals that are notorious to health and environment (e.g., $\mathrm{Hg}, \mathrm{Cr}, \mathrm{Cd}, \mathrm{Pb}$ ) motivate the current interests in graphene heavy metal sensors. ${ }^{[186,187]}$ Scientists have functionalized the surface of exfoliated graphene with a selfassembled monolayer of 1-octadecanethiol and applied it for $\mathrm{Hg}^{2+}$ sensing. ${ }^{[188]}$ Due to the high binding affinity of the thiol groups of 1-octadecanethiol to $\mathrm{Hg}^{2+}$, the sensor achieved a detection limit of $10 \mathrm{ppm}$. DNAzyme aptamer decoration, on the other hand, leads to $\mathrm{Pb}^{2+}$ detection down to $37.5 \mathrm{ng}$ $\mathrm{L}^{-1}$ with prototype device demonstrated using real blood samples. ${ }^{[189]}$ Instead of using exfoliated graphene, solutionprocessable rGO has also been developed for a low-cost, 
(a)

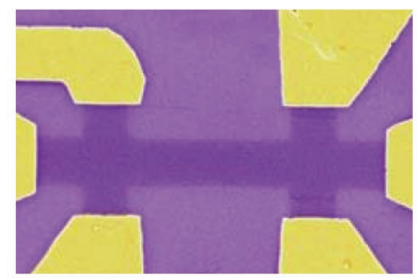

(b)

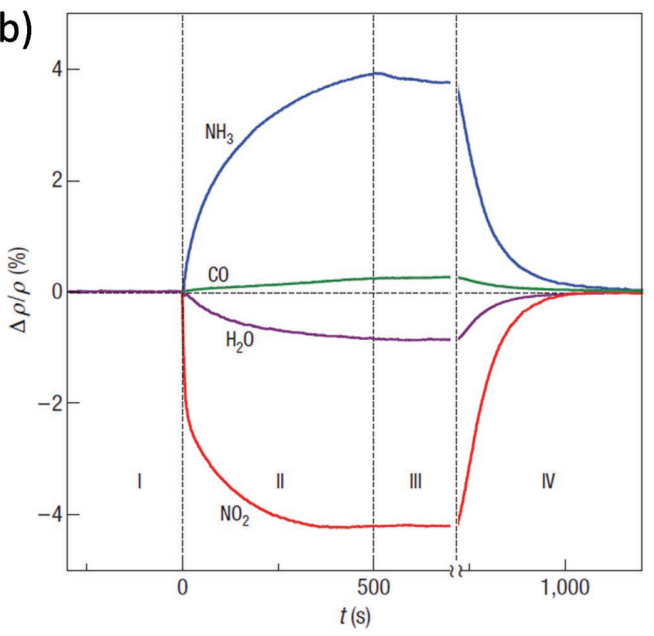

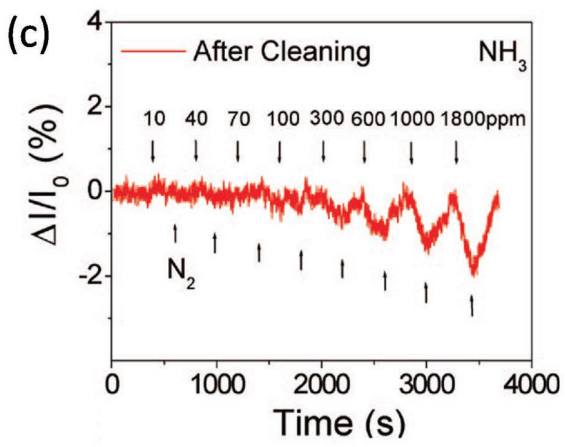

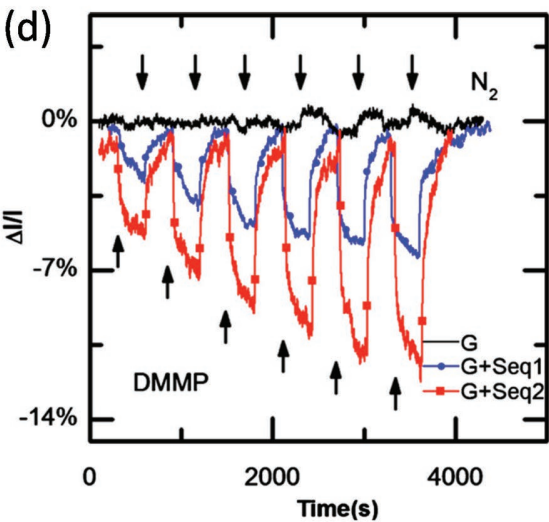

Internet of nano-things

(e)
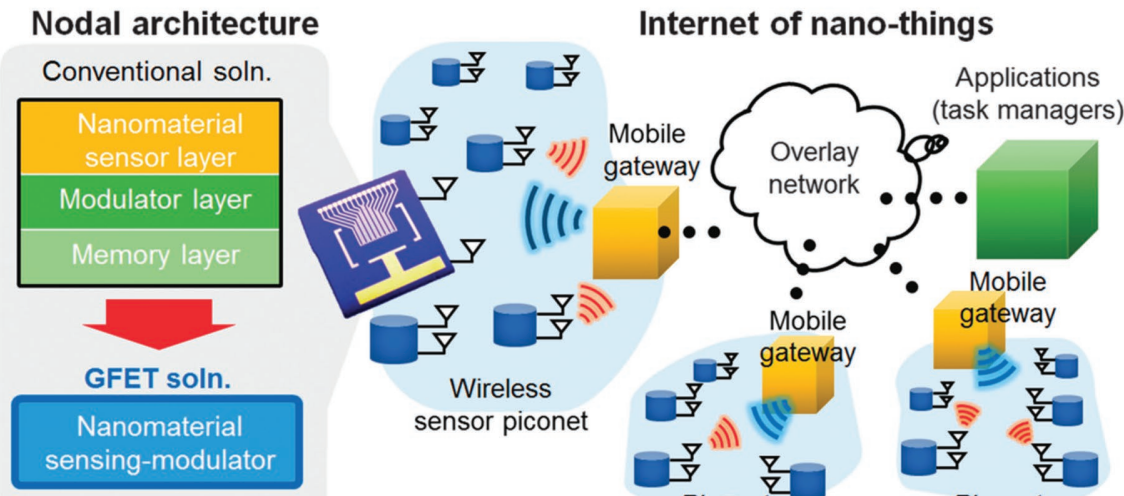

Piconet

Piconet

Figure 5. a) Colored SEM of a graphene (purple) Hall bar device. The scale bar is given by the width of the Hall bar, which is $1 \mu \mathrm{m}$. Yellow color corresponds to metal electrodes evaporated on the $\mathrm{SiO}_{2} / \mathrm{Si}$ substrate. b) Relative changes in resistivity $(\Delta \rho / \rho$, in percentage) caused by the exposure of graphene to various gases diluted at $1 \mathrm{ppm}\left(\mathrm{NH}_{3}\right.$ : blue curve, $\mathrm{CO}$ : green curve, $\mathrm{H}_{2} \mathrm{O}$ : purple curve, $\mathrm{NO}_{2}$ : red curve). Here the positive (respectively negative) changes in the $\Delta \rho / \rho$ curves in blue and green (purple and red, respectively) indicate electron (hole) doping. Reproduced with permission. ${ }^{\left[{ }^{5}\right]}$ Copyright 2007, Nature Publishing Group. c) Relative changes in current $\left(\Delta I / I\right.$, in percentage) of annealed exfoliated graphene $\left(1 \mathrm{~h}\right.$ at $400{ }^{\circ} \mathrm{C}$ in $\mathrm{Ar} /$ $\mathrm{H}_{2}$ atmosphere) towards the detection of ammonia vapors. The lower amount of defects in the clean graphene device yields smaller changes in the sensing response, even for ammonia concentrations as high as $1000 \mathrm{ppm}$. Lower arrows indicate when the sensor device was flushed with a pure $\mathrm{N}_{2}$ carrier gas. Reproduced with permission. ${ }^{[1]}$ Copyright 2009. American Chemical Society. d) Relative changes in current $(\Delta l / I$, in percentage) caused by the exposure of clean graphene device (black data) and ssDNA functionalized graphene devices (Seq1 in blue: 5'-GAG TCT GTG GAG GAC GTA GTC-3', Seq2 in red: 5'-CTT CTC TCT TCA TCT TTG TCA AAC-3') to dimethylmethylphosphonate (DMMP) vapor at concentrations of 20, 40, 60, 80, 100 , and 120 ppm. Upper arrows indicate when the sensor cell was purged with inert $\mathrm{N}_{2}$ gas. Reproduced with permission. ${ }^{[29]}$ Copyright 2010 , AlP Publishing LLC. e) A conceptual nodal architecture of an internet of things consisting of nanomaterial sensing modulators made of wireless GFET solutions. Open access. ${ }^{[192]}$

scalable fabrication of GFET sensors. ${ }^{[190]}$ By functionalizing the rGO with specific metal ion binding proteins or gold nanoparticle, nanomolar concentrations of $\mathrm{Hg}^{2+}, \mathrm{Cd}^{2+}$, and $\mathrm{Pb}^{2+}$ were detected. ${ }^{[186]}$ Impressive prospective has also been shared for smart graphene chemical sensors (which can be, in principle, wireless and wearable ${ }^{[191]}$ ) with low maintenance cost and low energy consumption for real-time, event-based monitoring in pervasive healthcare internet-of-things applications (Figure 5e). ${ }^{[192]}$ The selectivity of the detection, however, was not always thoroughly studied or understood in the 
reports representing a major challenge in the field (and for GFET biosensors in general). ${ }^{[193]}$

\subsection{GFET Glucose, DNA and Protein Biosensors}

Glucose detection has been an enduring topic since it directly reflects the metabolism condition and inspection of the chronic diabetes (the physiological concentration range of glucose detection falls in $\approx 2-20 \mathrm{mM}$ in serum). ${ }^{[194,195]}$ Recently, the perspectives for non-invasive portable point-of-care (POC) glucose sensors imposed imperative need for biosensors with high sensitivity and reliability, as the glucose levels in body fluids (saliva, tears, urine, etc.) are much lower $(\approx 0.01 \%)$ than that in plasma. ${ }^{[196]}$ Conventional electrochemical glucose sensors are not sensitive enough for these applications. On the other hand, detection of glucose with the aid of electronic devices such as GFETs can reach high sensitivity down to $\mathrm{nM}$ concentrations (see Table 2), which are sensitive enough for non-invasive glucose detections in body fluids. It is noteworthy that GFET glucose biosensors have also been fabricated on flexible supports such as PET (polyethylene terephthalate) $)^{[197]}$ and silk fibroin. ${ }^{[198]}$ In a continuous effort to achieve flexible and cheap biosensors, recently a GFET was implemented within a 2D paper network for practical glucose detection

(a)

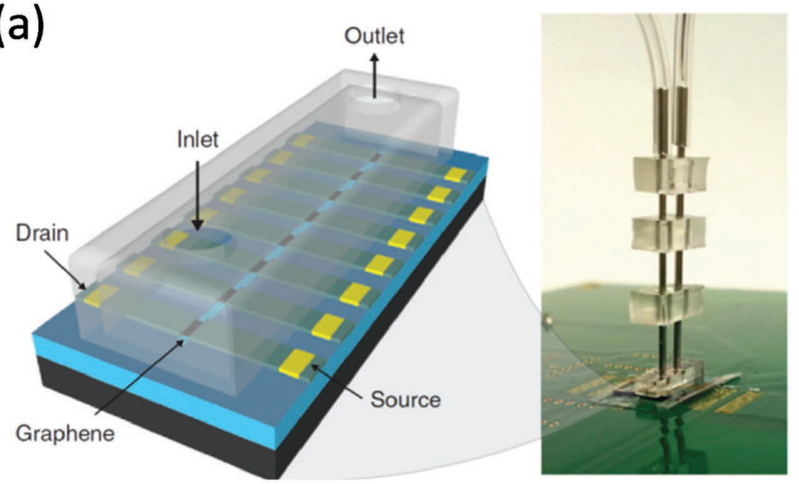

(b)

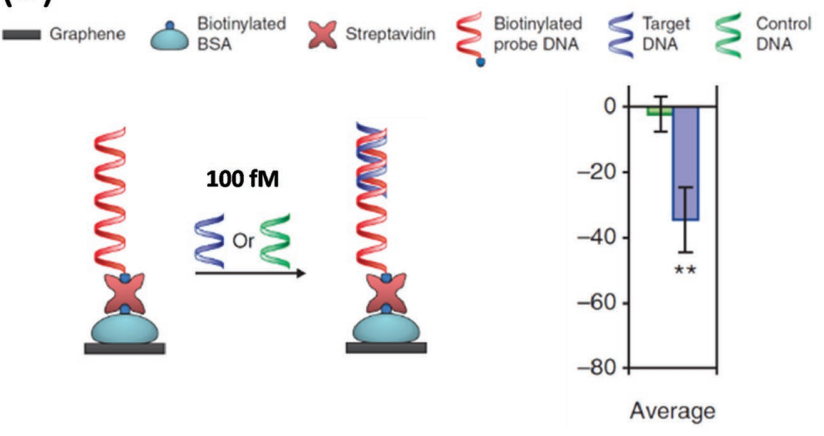

$(\approx 1.25 \mathrm{mM}) \cdot{ }^{[199]}$ Interestingly, using a PANI/Nafion-bilayer graphene modified gate electrode, researchers could also determine glucose level in saliva with a detection limit of $30 \mathrm{nM} .^{[200]}$ We would like to note here that, despite the recent achievements in GFET glucose biosensor development, more research is still needed to achieve a highly sensitive and reliable platform for home use or portable application. These challenges include modifying enzyme activity for improved performance, developing methods to reliably bind them to the graphene surface, and incorporating the (functionalized) GFET into a microfluidic device. ${ }^{[194]}$

Intrinsically, DNA and proteins, which contain aromatic groups, can bind onto graphene surface through $\pi-\pi$ interaction or simply by hydrophobic interactions (see Table 1). ${ }^{[98]}$ In one of the first GFET DNA sensors, single-stranded DNA (SSDNA) was anchored on the surface of GFETs and used to detect complementary DNA strands through hybridization. ${ }^{[9]}$ It has also been demonstrated that the GFETs were capable of detecting DNA hybridizations with single-base specificity. ${ }^{[201]}$ One step further from a single device to multiplexed DNA arrays, CVD GFET sensor arrays were manufactured (Figure 6a) and acted as both an electrophoretic electrode for site-specific DNA immobilization (not shown here) and hybridization detection down to $100 \mathrm{fM}$ (Figure $6 \mathrm{~b}){ }^{[22]}$ GFETs were also capable of distinguishing the conductance signature upon adsorption of the four different DNA
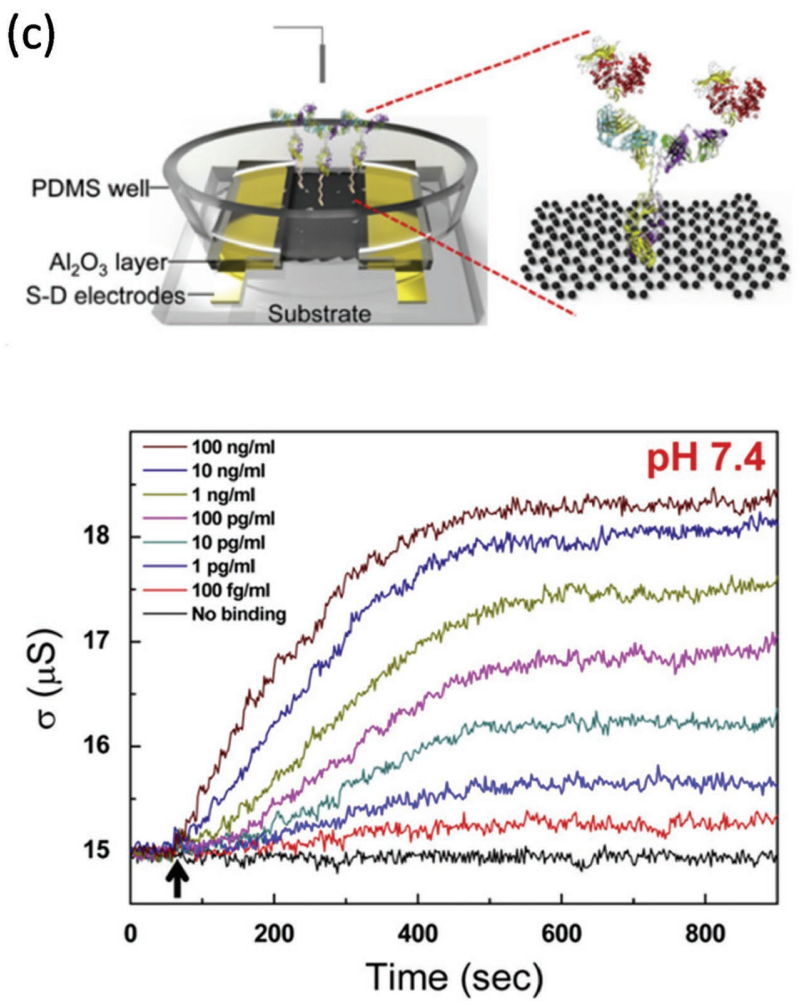

Figure 6. a) Schematics and image of an 8-graphene-electrode/FET array that sits on a printed circuit board with a microfluidic channel on top. b) $100 \mathrm{fM}$ hybridization (blue) and control (green) DNA detection. The sensing signals (hybridization DNA ** in blue and control DNA in green) were averaged across all eight GFETs. Reproduced with permission. ${ }^{[22]}$ Copyright 2014, Nature Publishing Group. c) Upper panel: illustration of rGO FET immunosensor with Pt reference electrode in the analyte solution. The $\mathrm{rGO}$ surface is functionalized with prostate specific antigen monoclonal antibody (PSAmAb). The zoom-in indicates the immunoreaction between the antibody (PSAmAb) and its antigen (PSA/ $\alpha 1$-antichymotrypsin, PSA-ACT complex). Lower panel: plot of the conductance versus time for antibody (PSAmAb) functionalized rGO FET upon the binding of its antigen (a PSA-ACT complex). Experiments were carried in a PBS buffer solution at $\mathrm{pH}=7.4$. The black arrow indicates the injection of the antigen (PSA-ACT complex) at increasing concentrations. Reproduced with permission. ${ }^{[78]}$ Copyright 2013, Elsevier. 
nucleobases due to the different interface dipole field. ${ }^{[97]}$ The same study concluded that the sensing of single nucleotide with graphene is feasible even without DNA amplification (amplification of DNA showed a detection limit of $50 \mathrm{aM}$ using rolling circle amplification $^{[100]}$ ). Another possibility for graphene-based DNA sensors, ${ }^{[202]}$ is to configure a graphene nanoribbon FET with a nanopore and to probe the subtle differences in the conductance as the negatively charged DNA molecules translocating through the nanopore. ${ }^{[203]}$ Interestingly, single-stranded DNA can also be used as a sensitizing agent to selectively probe various gases. ${ }^{[29]}$ Contrarily to the inert sensing behavior of clean GFETs to various gas vapor such as dimethylmethylphosphonate (DMMP, black line, Figure 5d), ssDNA decorated GFET showed selective sensing responses to vapor of DMMP (blue and red lines indicate different sensing responses when the GFETs were functionalized with two DNA sequences). ${ }^{[29]}$ We note here that, as a functional DNA or RNA molecule selected in vitro to bind pre-selected analytes (organic and inorganic molecules and proteins) with high affinity and specificity, aptamers also represent a versatile toolbox for producing novel graphene sensors. ${ }^{[171,204,205]}$

GFETs have also been widely reported for protein detection. In an earlier study, bovine serum albumin (BSA) nonspecifically adsorbed on a graphene surface can be detected at 300 pM concentration. ${ }^{[10]}$ Separately, selective detection of immunoglobulin E (IgE) can be realized by functionalizating graphene with aptamers (through probing the charges the IgE brings as it binds to the aptamers) $\cdot{ }^{[171]}$ One of the important expectations/outcomes of a highly sensitive and selective bioGFET is to analyze biomarkers for especially point-of-care applications. ${ }^{[65-67]}$ As an example, femtomolars of prostate specific antigen $/ \alpha 1$-antichymotrypsin (PSAACT) - a complex biomarker in prostate cancer diagnosis - could be detected using a rGO FET (Figure 6c, lower panel) by functionalizing the graphene surface with PSA monoclonal antibody (PSAmAb) (Figure 6c, upper panel). ${ }^{[78]}$ Attomolar level detections have also been reported occasionally ${ }^{[205]}$ but such significantly improved sensitivities were not always consistent with the more generally observed nM-pM detection limit, ${ }^{[66,67]}$ suggesting that more dedicated efforts are still needed to improve the reliability and the reproducibility of the sensing response. ${ }^{[206]}$ This brings us back to some of the fundamental issues associated with graphene-based BioFETs (and BioFETs in general) as eventually, after carefully designing and controlling every steps of a GFET, we will be looking at the Debye screening effects as an obstacle to achieve ultimate detection of a relatively large biomolecule in physiological conditions as previously discussed in Section 2.5 and will be further reviewed in the next Section 5.2. Alternatively, the detection of biomolecules can also be achieved by monitoring $\mathrm{pH}$ changes during adsorption, thus circumventing the Debye screening effects as proton is negligibly small. ${ }^{[207]}$ Along this line, GFETs were applied as $\mathrm{pH}$ sensors to detect alanine aminotransferase (ALT) in the concentration range of $10-100$ unit $L^{-1}$ by monitoring the generation of protons during the enzymatic reaction between L-alanine/ $\alpha$-ketoglutarate and ALT. ${ }^{[208]}$

\subsection{GFET Biological Cellular Sensors}

Recently, as a part of the Europe's Graphene Flagship - a €1 billion project aims to bring graphene from innovative laboratory researches into commercial applications, scientists interfaced graphene to neurons directly, while retaining the integrity of these vital nerve cells. ${ }^{[148]}$ In fact, stimulating and recording extracellular potentials (or even intracellular potential using branched transistors ${ }^{[209]}$ ) from neurons is one of the hallmark of modern bioelectronics. Graphene can serve not only as conductive electrodes to transduce stimuli into the cells, but also as the conductive channel of GFETs to monitor the presence and activity of the cells. ${ }^{[99]}$ Figure 7 a illustrates a cell-transistor measurement, ${ }^{[68]}$ where a cell is located on the graphene surface. A constant bias voltage is applied to the drain and source electrodes (in gold yellow), bridged by a graphene conductive channel (in pink). The current in the graphene channel is amplified and monitored in real time. Any local electrochemical potential change triggered by the action potential of the cell leads to a modulation of the source-drain current in graphene (due to the field effect). A separate study combined GFETs (exfoliated graphene) and silicon nanowire FETs to probe single electrogenic cardiomyocyte cell (Figure 7b). ${ }^{[176]}$ The GFETs yielded well-defined current peaks (extracellular signals) with signal-to-noise ratio (SNR) routinely above four, which is comparable to that of the nanowire FETs (Figure 7c). ${ }^{[176]}$ Narrow GFETs $(\approx 2 \mu \mathrm{m} \times 3 \mu \mathrm{m})$ exhibit similar peak-to-peak widths as the nanowireFET $(\approx 100$ times smaller per area). But GFET devices with large size $(\approx 20 \mu \mathrm{m} \times 10 \mu \mathrm{m})$ detect an average of the extracellular potential from beating cells and yield a broadened peak-to-peak signal width.

Arrays of GFET have been developed to monitor tissue-like specimens. ${ }^{[210]}$ Densely packed cardiomyocyte-like HL-1 cells were cultured on the surface of graphene arrays (a fluorescence image of the cells is shown in Figure 7d). The propagation of the action potentials across the beating cellular network is monitored by recording the current flowing through the transistor array (Figure 7e). In the current trace, spikes from the different GFETs depict the variations in the graphene-cell coupling and the propagation of the action potentials across the HL-1 cell network. Furthermore, these GFETs exhibit a SNR better than ten. Even at its early stage of development, such SNR performance equals (or even surpasses) that of well-established techniques like the microelectrode arrays (MEAs) and the planarFET ${ }^{[211]}$ and nanowireFET. ${ }^{[176]}$

In order to provide a control over the specific voltage-gated ion-channels in the cell membrane ${ }^{[212]}$ (and thus the action potential of the cell), patch-clamping experiments were combined with GFETs (Figure 7a), where a patch pipette containing an inner electrode was manually introduced into the inner part of a single HEK-293 cell. Because the HEK-293 cell were genetically modified so that the membrane contains only $\mathrm{K}^{+}$channels, ${ }^{[213]}$ the opening and closing of these ion channels could be unambiguously identified by monitoring the current across the membrane with the patch electrodes. ${ }^{[68]}$ Simultaneously, signals from the GFET could be attributed to these $\mathrm{K}^{+}$channels and very good SNR (up to fifty) could be achieved by repeating the same process multiple times. ${ }^{[68]}$ Besides the action potential, GFET can also measure the electromechanical coupling between rGO microsheets and - for example - yeast cells (Figure 7f). ${ }^{[147]}$ The flexible rGO layer responses electrically to the change in cell volume when exposed to different alcohols due to a strong mechanical coupling. By monitoring the 

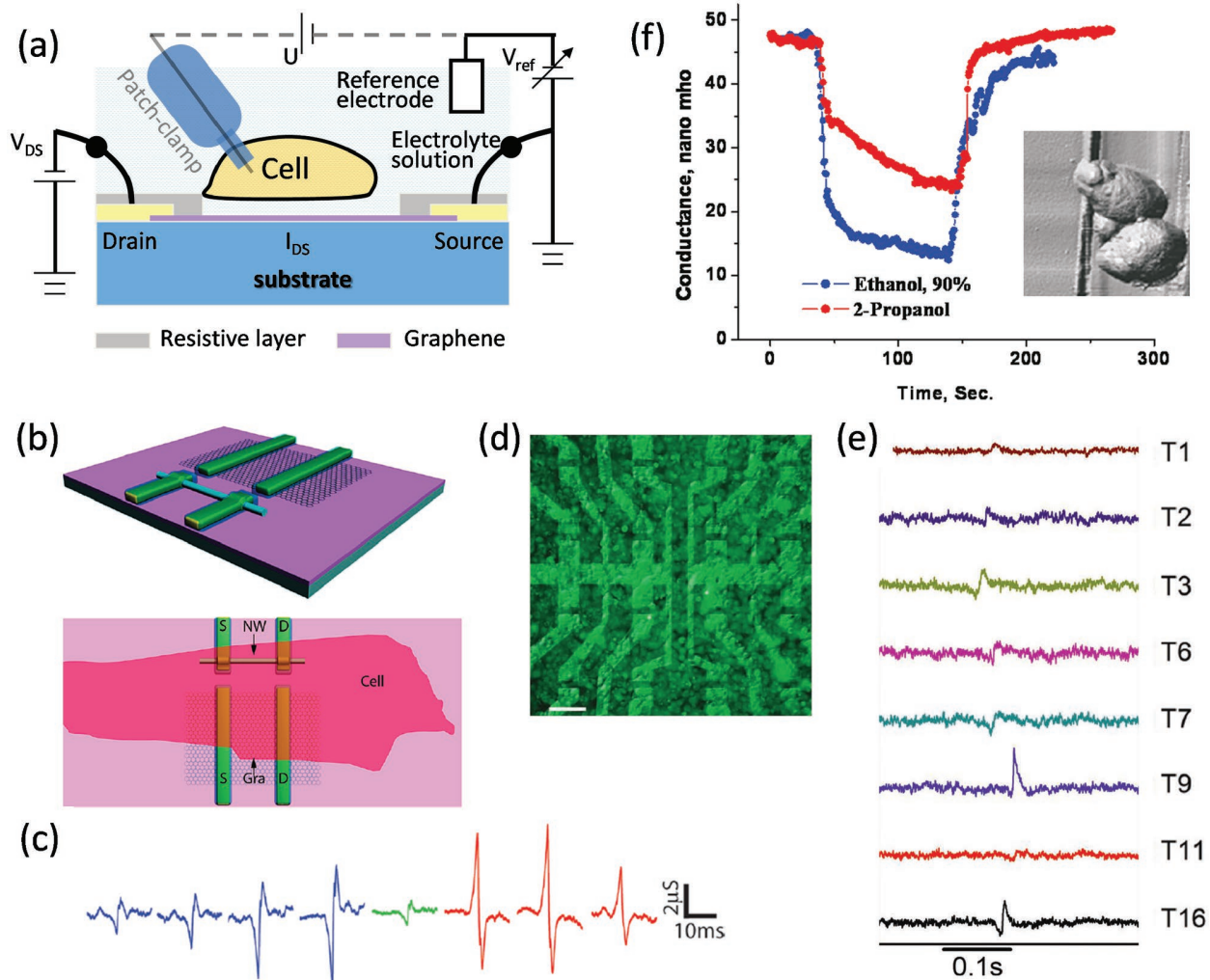

(c)

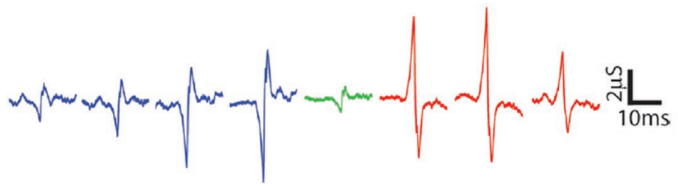

Figure 7. a) Schematic of a single cell coupled to a GFET combined with a patch-clamp electrode setup. b) Top: illustration of two sensors, one incorporating a SiNW and a second using graphene as the sensing element. Bottom: illustration of the chip design incorporating graphene and SiNW devices, highlighting the relative size of a cardiomyocyte cell with respect to the device dimensions. c) The representative detection peaks in blue were recorded at the p-type polarity of the GFET, red peaks represent recorded traces at the n-type device polarity, and the green peak was recorded near the CNP of the GFET. Reproduced with permission. ${ }^{[176]}$ Copyright 2010, American Chemical Society. d) A fluorescent optical image of a GFET array with stained cells. Scale bar: $100 \mu \mathrm{m}$. e) Time-dependent current recordings of eight transistors from one array (marked from T1 to T16). Reproduced with permission. ${ }^{[210]}$ Copyright 2011, John Wiley and Sons. f) Conductance versus time plots of a rGO FET upon exposure of the (rGO covered) cells to ethanol and 2-propanol solutions. Inset: AFM image of the surface of a cell deposited between Au electrodes on a silicon dioxide chip. Reproduced with permission. ${ }^{[147]}$ Copyright 2011, American Chemical Society.

dynamic electrical response of $\mathrm{rGO}$, it was possible to differentiate between ethanol, 2-propanol, and water, each inducing different physiological stress response on the cell.

Based on the state-of-the-art of GFET cellular sensors, graphene and graphene-related materials have confirmed their suitability for monitoring the activity of cells with excellent SNR (both electrically and mechanically). Looking ahead, new advancements could reside in the development of flexible GFET cellular sensors, ${ }^{[214]}$ by combining the outstanding electronic performances of GFET with the high flexibility of graphene. ${ }^{[215]}$ In such a flexible bioelectronic platform, individual cells in a network can be addressed via electrical interfaces, which could potentially lead to advanced learning circuits, neuron-implants, and neuroprosthesis that could potentially replace damaged nervous tissue for treating brain and paralysis diseases. ${ }^{[148]}$

\subsection{Graphene-based Electrochemical (GEC) Biosensors}

In liquid-gated GFET biosensors, the electrical current is confined transversely in the graphene conductive channel. Any electrochemical current vertically flowing between the graphene channel and the liquid gate (through the electrolyte solution), is regarded as a spurious signal and limits the performances of gate controlled GFET devices. ${ }^{[12]}$ This electrochemical current is - however - at the basis of graphene electrochemical (GEC) biosensors, which are complementary to GFETs. ${ }^{[216]}$ In this regard, it is necessary to understand the construction as well as the working principle of a GEC sensor, in order to fully appreciate the operation of a GFET. Specifically, the sensing principle of a GEC sensor roots on the electrochemical transfer current between the redox active biomolecules in the solution-phase and graphene surface.

Similarly to GFETs, the GEC uses the surface of graphene as the major sensing element. Until now, the majority of GECs uses graphene dispersions (usually nanosheets of chemically functionalized graphene) deposited on conductive electrodes. ${ }^{[217,218]}$ These graphene dispersions with large surface to volume ratio - in contrast with mono- or bilayer graphene sheets employed in GFETs - contain more defective areas. These defects enhance the density of electronic states (DOS) of graphene dispersions, which favors the electron transfer between the graphene materials and the redox biomolecules, and thus yielding a higher sensitivity. ${ }^{[219]}$ For 
example, reduced graphene nanowalls (rGNW) with large amount of sharp edges have been deposited vertically via electrophoresis on a graphite electrode to detect double-stranded DNA (dsDNA) with an impressively wide detection concentration range of $0.1 \mathrm{fM}-10 \mathrm{mM} .^{[218]}$

The sensitivity of the abovementioned GEC biosensors resides in the defects of graphene. Functionalizations of these defects with electrochemical catalysts lead to further improved sensitivity and selectivity for the detection of a wide range of molecules, namely glucose, ${ }^{[220]}$ cholesterol, ${ }^{[221]}$ DNA, ${ }^{[222,223]}$ proteins, ${ }^{[224]}$ and even living cells. ${ }^{[103,225]}$ To functionalize graphene, most typical catalysts are composed of enzymes, ${ }^{[226]}$ metal nanoparticles, ${ }^{[227]}$ and polymers, ${ }^{[228]}$ to name a few. In fact, to a large extent the functionalization of a GEC is similar to that of a GFET. Advantageously, covalent functionalization often results in dramatically enhanced DOS in graphene facilitating higher electron transfer rate. ${ }^{[219]}$ Non-covalent functionalization, however, has the advantage to retain the excellent electrical properties of graphene, and, to a certain extent, limit the possible charge transfer across the interface, and thus favoring the GFET biosensors. Drop-casting deposition ${ }^{[223]}$ is one of the most widely adopted methodologies to fabricate GEC biosensors with functionalized graphene dispersions. Such graphene dispersions, however, usually contain a mixture of mono-, bi-, few layer graphene flakes with uncontrolled and even unknown defect, impurities, or chemical functionalities. In order to unambiguously address the electrochemical properties of graphene, the difference in the electrochemical activity of the edge and the basal plane of graphene have been carefully studied (Figure 8a). ${ }^{[229]}$ Graphene edges (with current density $\mathrm{j}=0.11 \mathrm{~A} \mathrm{~cm}^{-2}$ ) exhibit larger electrocata-

lytic properties, while the basal plane $\left(j=2.2 \times 10^{-4} \mathrm{~A} \mathrm{~cm}^{-2}\right)$ is relatively inert (Figure $8 \mathrm{~b}$ ). Previous studies carried out on clean graphene monolayer with a well-defined surface area, ${ }^{[230]}$ on free-standing graphene samples over a nanopore, ${ }^{[231]}$ and on graphene and graphite step edges using scanning electrochemical microscopy (SECM), ${ }^{[232,233]}$ confirmed this trend that edges are electrochemically more active than the basal plane. ${ }^{[125]}$ In combination with Raman spectroscopy (Figure 8c), SECM is able to quantitatively correlate the defect density of graphene with its localized electrochemical activity (Figure 8d), ${ }^{[233]}$ providing new possibilities to systematically study the electrochemical properties of graphene. The correlation indicates that the electrochemical activity first increases with the defect density (in line with earlier reported higher reactivity for covalent derivatization $^{[122,234]}$ ), and then decreases when "defective" graphene sheet loses its structure integrity (i.e., presumably when the aromaticity of graphene is totally lost). As a perspective, a GFET biosensor can in principle be combined with a GEC biosensor we described here in a same device, and thus providing a fully complementary sensing platform to study both the electrostatic charge of the biomolecules but also the charge transfer during redox reaction at the graphene surface. Such device configuration has already been realized in organic electrochemical transistors with graphene-modified gate electrodes, which was proved to significantly improve the selectivity of the organic electrochemical transistors for dopamine detection. ${ }^{[154]}$ 


\section{Perspectives and Conclusions}

GFET is a young member of the huge family of current biosensors based on optical transduction and/or electrical transduction principles, but it has already opened a totally new, very promising range of options for biochemical sensing. Indeed, the reliability and reproducibility of GFET biosensors were not always thoroughly studied or understood in the reports, representing a major challenge in the field ${ }^{[193]}$ that, in principle, could be solved via reliable processing of device fabrication and rational design of graphene surface functionalization. We believe that the future of GFET based biosensor exploration should focus on the unique physical and chemical properties that graphene offers, ${ }^{[235]}$ in the direction to improve even further its sensitivity with ever-demanding reliability and reproducibility. In the following, we will have an in-depth look at the benefit by reducing possible competing sensing reactions to preserve the highest possible biological signal at graphene surface. Particularly, we will discuss current progresses on high frequency biosensors based on nanostructures including graphene materials, which suggest that the Debye length limitations could be overcome in physiological conditions without any special design or engineering of the receptor molecules and the sensor environments. We expect these perspectives will prompt GFET's future development with potential to revolutionize the fields ranging from neuronal sensing to point-ofcare medical diagnosis. ${ }^{[65-67,192]}$

\subsection{Graphene as a Chemically Inert Surface: Towards an Ultimate Biosensor}

In 2011, Ion Torrent by Life Technologies (USA) directly translated chemically encoded information in genomes (A, C, G, T) into digital information $(0,1)$ on a semiconductor chip, and thus being able to commercialize the first low-cost integrated semiconductor device based on Si technology for non-optical genome (DNA) sequencing. ${ }^{[207]}$ The DNA sequencing is achieved by detecting the $\mathrm{pH}$ changes induced by protons that are released when nucleotides (dNTP) are incorporated on the growing DNA strands. In fact, $\mathrm{pH}$ sensors are the only ion-sensitive FET sensors that have been successfully commercialized due to the highly sensitive $(\approx 60 \mathrm{mV} / \mathrm{pH})$ and selective nature of the highly abundant hydroxyl $(-\mathrm{OH})$ terminal groups on the oxide surface $\left(\mathrm{SiO}_{2}, \mathrm{Al}_{2} \mathrm{O}_{3}, \mathrm{HfO}_{2}, \mathrm{Ta}_{2} \mathrm{O}_{5}\right.$, etc.) towards protons. ${ }^{[42]}$ Although Si ion-sensitive FETs can also probe and detect biomolecules directly, ${ }^{[236]}$ no commercial biological ion-sensitive FETs has been released so far. In fact: i) such devices are more capable of detecting small molecules such as protons, as the Debye screening effect limits the charge detection of large biomolecules (as discussed in Section 5); ii) the very high density of the hydroxyl $(-\mathrm{OH})$ groups diminishes the sensing response of the $\mathrm{Si}$ ion-sensitive FET to biomolecules. That is, upon the binding of target biomolecules onto the receptors immobilized on the surface of a $\mathrm{Si}$ ion-sensitive FET, the protonation $\left(-\mathrm{OH}_{2}{ }^{+}\right)$or deprotonation $\left(-\mathrm{O}^{-}\right)$of the nearby unpassivated $-\mathrm{OH}$ groups ${ }^{[42]}$ (a full passivation of the surface $-\mathrm{OH}$ groups by receptors is difficult ${ }^{[237]}$ ) will buffer and significantly screen/hinder the sensing response of the $\mathrm{Si}$ ion-sensitive FET to the charged biomolecules. ${ }^{[236]}$ In this regard, beside the above discussed well-known advantages (such as high mobility, low intrinsic electrical noise, large surface to volume ratio and stability) the fact that graphene possesses a surface that is free of dangling bonds (-OH groups, for example), represents another significant advantage and unprecedented opportunities over Si ion-sensitive FETs for biosensing applications.

This screening/interference effect can be understood by considering the following iterative sensing steps: i) positively charged target biomolecules attaching to the receptors cause a change in the total surface charge: $+\Delta Q_{0}$ (biosensing signal); ii) the change in the total surface charge initiates a redistribution of the proton concentration near the sensor surface due to Boltzmann distribution in the electrolyte environment; ${ }^{[42]}$ iii) the change in the proton concentration at the surface will, in turn, induce a negative change in the surface charge $-\Delta Q_{01}$ due to the protonation/deprotonation of the $-\mathrm{OH}$ groups, which will compensate the aforementioned sensing signal $+\Delta Q_{0}$. In an extreme case, this compensation will be complete if the sensor surface has a Nernstian $\mathrm{pH}$ response ${ }^{[238]}$ (indicating very high density of unpassivated -OH groups). Consequently, there will be no sensing response because any changes in the surface charge $+\Delta Q_{0}$ due to positively charged target biomolecules attached to surface immobilized receptors are totally screened by the protonation/deprotonation reactions. In fact, a reduced biosensing signal can be measured if the compensation is not complete. ${ }^{[239]}$ In our opinions, this screening effect has caused many problems for FET-type of biosensing applications (sometimes even without noticing), where the surface functionalization started with a surface of very high -OH group density. In the contrary, the highest sensitivity is reached, if the density of the surface $-\mathrm{OH}$ groups is zero (except if one aims to sense the $\mathrm{pH})$. This is the case of ideal graphene. Hence, if graphene is used as the sensing surface and the conducting channel in an ion-sensitive FET at the same time, an ultimate sensor could be realized.

\subsection{Overcoming the Debye Length Limitations with Radio-frequency (RF)-operated GFETs}

GFETs are primarily sensitive to the charges carried by the biomolecules adsorbed on top of the graphene surface. In previous Section 2.5, we reviewed that FET based biosensors could suffer from the ionic screening due to mobile ions present in the solution, known as Debye screening effect. ${ }^{[73]}$ As the charges are heavily screened, they can hardly be detected if the distance between the charged biomolecules and graphene surface exceeds several times the Debye length, which is about $0.7 \mathrm{~nm}$ at physiological conditions. Possible routes to circumvent the Debye screening effect include short antibody design, porous polymer incorporation, and ex situ measurement in low ionic strength buffers (see Section 2.5)..$^{74-77,80]}$ These approaches, however, also impose limitations on the biodetection and it is highly desirable to develop a straightforward methods to overcome the Debye screening: ${ }^{[46]} 1$. without any special design or engineering of the receptor molecules and the sensor environments, and 2. in physiological conditions to facilitate in situ, real-time biosensing. 
(a)
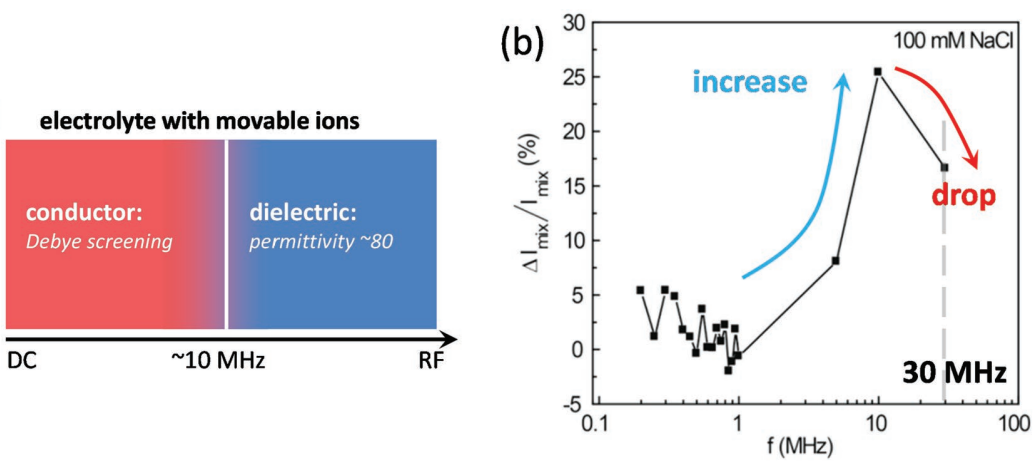

Figure 9. a) The Debye screening effect dominates at DC and frequencies lower than $\approx 10 \mathrm{MHz}$. At higher frequencies, the electrolytes behave as dielectrics. b) Relative changes in mixing current $\left(\Delta \mathrm{I}_{\text {mix }} / \mathrm{I}_{\text {mix }}\right.$, in percentage) of a carbon nanotube-based streptavidin-biotin sensor as a function of frequency at $100 \mathrm{mM} \mathrm{NaCl}$ solution. Reproduced with permission. ${ }^{[46]}$ Copyright 2012, American Chemical Society.

Theoretically, improved sensitivity is expected at high frequencies using a measuring strategy that overcomes the ionic screening effect. This improvement is because at RF/microwave frequencies the ions in the electrolyte lag behind the alternating current (ac) electric field due to the viscosity of the solution. ${ }^{[40]}$ As a result, the Debye screening is canceled and the buffer solution can be regarded as a pure dielectric at high frequencies (much larger than $10 \mathrm{MHz}$, see Figure 9a). ${ }^{[40]}$ Hence the displacement of water from the interface by a lower dielectric constant such as a biomolecule can be detected/ transduced as the output signal of the RF sensors, which ultimately relates to the chemical structure (i.e., dipole moment) of the biomolecule. ${ }^{[46]}$ Recently, in an attempt to detect beyond the Debye length, carbon nanotube sensors were operated at frequencies up to $\approx 10 \mathrm{MHz}$. ${ }^{[46]}$ The sensing response, defined as the relative change of the measured mixing current $\Delta \mathrm{I}_{\text {mix }} /$ $I_{\text {mix }}$ of the sensor, increases for increasing the measurement frequencies (up to $10 \mathrm{MHz}$, Figure 9b, blue arrow) due to a continuous reduction of the Debye screening effect. However, an unexpected drop in the sensing response at $30 \mathrm{MHz}$ was observed (Figure 9b, red arrow). In fact, at frequency smaller than $10 \mathrm{MHz}$ the ions in the aqueous solution (at physiological conditions) can still follow the electrical signal. ${ }^{[40]}$ As a result, the charged biomolecules are still screened (at least partially) by the movable ions in the buffer solutions (Figure 9a). In order to unambiguously test whether GFET operated at high frequency can overcome the Debye screening limitation, further research at frequencies above $10 \mathrm{MHz}$ would still be highly desired.

In fact, owing to its exceptional high mobilities, graphene is potentially suited for high-frequency applications. ${ }^{[15]}$ For instance, high-frequency GFETs with an intrinsic cut-off frequency of above $300 \mathrm{GHz}$ have been demonstrated, which outperforms the best silicon FETs with similar gate lengths. ${ }^{[240]}$ However, our knowledge of the high-frequency (much higher than $10 \mathrm{MHz}$ ) properties of GFETs in the presence of the electrolyte (in direct contact with the graphene layer) is very limited. As an attempt to forward our understanding of the RF properties of graphene, especially with regards to sensing in a liquid environment, the operation of an electrolyte-gated GFET at $\mathrm{RF} /$ microwave frequencies $(\approx 2-4 \mathrm{GHz})$ has been achieved in a recent study. ${ }^{[241]}$ Owing to the wide bandwidth, the graphene sensor fields. ${ }^{[192]}$

RF device also enables ultrafast measurements at nanosecond time-resolved. ${ }^{[241]} \mathrm{We}$ note here that AC electric field also exerts great influences on the fluid and results in micro-nano electrokinetic phenomena like electroosmosis and especially dielectrophoresis, ${ }^{[242]}$ which could, in principle, be adopted to manipulate, sort, and concentrate different types of nanoparticles and biomolecules at the strongest field range to enhance further the detection limit. Indeed, the development of RF sensors is still in the early stage, but already reveals great potential for a wide range of biochemical sensing applications. ${ }^{[243]}$ Sincerely, we hope that the perspective of RF graphene biosensors will open up new directions in research in the biomedical, environmental, and other high performance

To conclude, this article comprehensively reviews recent research efforts devoted to understanding the operation mechanisms of GFETs in electrolytic environments, and to functionalizing the graphene surface with particular recognition moieties to unlock its sensitivity potential. We provide a timely overview on the recent developments of gas sensors, protein and DNA biosensors, and biological cellular sensors, using the surface of graphene as the sensing element. We are convinced that graphene biochemical sensors hold great promise to meet the increasing demands on the sensitivity, especially by the recent progresses on operating GFETs at high frequencies suggesting that Debye screening can be overcome.

\section{Acknowledgements}

The research leading to this review has gratefully received funding from the European Research Council under the European Union's Seventh Framework Programme (FP/2007-2013)/ERC Grant Agreement n. 335879 project acronym 'Biographene', the FP7 funded DECATHLON Grant agreement n. 613908 'Development of Cost efficient Advanced DNA-based methods for specific Traceability issues and High Level On-site applications', the Netherlands Organization for Scientific Research (Vidi 723.013.007 and Veni 722.014.004), the Swiss National Science Foundation (P300P2_154557), and the China Scholarship Council (201406890016).

Received: July 8, 2016

Revised: August 18, 2016

Published online: November 29, 2016

[1] E. Stern, J. F. Klemic, D. A. Routenberg, P. N. Wyrembak, D. B. Turner-Evans, A. D. Hamilton, D. A. LaVan, T. M. Fahmy, M. A. Reed, Nature 2007, 445, 519.

[2] a) Y. Cui, Q. Q. Wei, H. K. Park, C. M. Lieber, Science 2001, 293, 1289; b) K. Besteman, J. O. Lee, F. G. M. Wiertz, H. A. Heering, C. Dekker, Nano Lett. 2003, 3, 727; c) C. Li, M. Curreli, H. Lin, B. Lei, F. N. Ishikawa, R. Datar, R. J. Cote, M. E. Thompson, C. W. Zhou, J. Am. Chem. Soc. 2005, 127, 12484; d) T. Cohen-Karni, R. Langer, D. S. Kohane, ACS Nano 2012, 6, 6541.

[3] a) A. P. F. Turner, Chem. Soc. Rev. 2013, 42, 3184; b) T. M. H. Lee, Sensors-Basel 2008, 8, 5535; c) M. Y. Shen, B. R. Li, Y. K. Li, Biosens. Bioelectron. 2014, 60, 101. 
[4] K. S. Novoselov, A. K. Geim, S. V. Morozov, D. Jiang, Y. Zhang, S. V. Dubonos, I. V. Grigorieva, A. A. Firsov, Science 2004, 306, 666

[5] F. Schedin, A. K. Geim, S. V. Morozov, E. W. Hill, P. Blake, M. I. Katsnelson, K. S. Novoselov, Nat. Mater. 2007, 6, 652.

[6] Y. Ohno, K. Maehashi, K. Matsumoto, Proc. Spie. 8031, 2011, 803121.

[7] I. Heller, S. Chatoor, J. Mannik, M. A. G. Zevenbergen, C. Dekker, S. G. Lemay, J. Am. Chem. Soc. 2010, 132, 17149.

[8] P. K. Ang, W. Chen, A. T. S. Wee, K. P. Loh, J. Am. Chem. Soc. 2008, $130,14392$.

[9] J. Ristein, W. Y. Zhang, F. Speck, M. Ostler, L. Ley, T. Seyller, J. Phys. D: Appl. Phys. 2010, 43, 345303.

[10] Y. Ohno, K. Maehashi, Y. Yamashiro, K. Matsumoto, Nano Lett. 2009, 9, 3318.

[11] Y. P. Dan, Y. Lu, N. J. Kybert, Z. T. Luo, A. T. C. Johnson, Nano Lett. 2009, 9, 1472.

[12] W. Y. Fu, C. Nef, O. Knopfrnacher, A. Tarasov, M. Weiss, M. Calame, C. Schonenberger, Nano Lett. 2011, 11, 3597.

[13] a) A. K. Geim, K. S. Novoselov, Nat. Mater. 2007, 6, 183 b) P. Avouris, Z. H. Chen, V. Perebeinos, Nat. Nanotechnol. 2007, 2, 605 .

[14] S. Das Sarma, S. Adam, E. H. Hwang, E. Rossi, Rev. Mod. Phys. 2011, 83, 407.

[15] F. Schwierz, Nat. Nanotechnol. 2010, 5, 487

[16] a) C. R. Dean, A. F. Young, I. Meric, C. Lee, L. Wang, S. Sorgenfrei, K. Watanabe, T. Taniguchi, P. Kim, K. L. Shepard, J. Hone, Nat. Nanotechnol. 2010, 5, 722; b) J. Baringhaus, M. Ruan, F. Edler, A. Tejeda, M. Sicot, A. Taleb-lbrahimi, A. P. Li, Z. G. Jiang, E. H. Conrad, C. Berger, C. Tegenkamp, W. A. de Heer, Nature 2014, 506, 349.

[17] L. Wang, I. Meric, P. Y. Huang, Q. Gao, Y. Gao, H. Tran, T. Taniguchi, K. Watanabe, L. M. Campos, D. A. Muller, J. Guo, P. Kim, J. Hone, K. L. Shepard, C. R. Dean, Science 2013, 342, 614.

[18] A. A. Balandin, Nat. Nanotechnol. 2013, 8, 549.

[19] Y. M. Lin, P. Avouris, Nano Lett. 2008, 8, 2119.

[20] A. N. Pal, A. Ghosh, Appl. Phys. Lett. 2009, 95, 082105.

[21] Z. G. Cheng, Q. Li, Z. J. Li, Q. Y. Zhou, Y. Fang, Nano Lett. 2010, 10, 1864.

[22] G. Y. Xu, J. Abbott, L. Qin, K. Y. M. Yeung, Y. Song, H. Yoon, J. Kong, D. Ham, Nat. Commun. 2014, 5, 4866.

[23] D. C. Elias, R. R. Nair, T. M. G. Mohiuddin, S. V. Morozov, P. Blake, M. P. Halsall, A. C. Ferrari, D. W. Boukhvalov, M. I. Katsnelson, A. K. Geim, K. S. Novoselov, Science 2009, 323, 610.

[24] R. R. Nair, W. C. Ren, R. Jalil, I. Riaz, V. G. Kravets, L. Britnell, P. Blake, F. Schedin, A. S. Mayorov, S. J. Yuan, M. I. Katsnelson, H. M. Cheng, W. Strupinski, L. G. Bulusheva, A. V. Okotrub, I. V. Grigorieva, A. N. Grigorenko, K. S. Novoselov, A. K. Geim, Small 2010, 6, 2877.

[25] J. T. Robinson, F. K. Perkins, E. S. Snow, Z. Q. Wei, P. E. Sheehan, Nano Lett. 2008, 8, 3137.

[26] K. Yang, L. Z. Feng, H. Hong, W. B. Cai, Z. Liu, Nat. Protoc. 2013, 8, 2392.

[27] a) E. Bekyarova, M. E. Itkis, P. Ramesh, C. Berger, M. Sprinkle, W. A. de Heer, R. C. Haddon, J. Am. Chem. Soc. 2009, 131, 1336; b) F. M. Koehler, W. J. Stark, Acc. Chem. Res. 2013, 46, 2297.

[28] F. M. Koehler, N. A. Luechinger, D. Ziegler, E. K. Athanassiou, R. N. Grass, A. Rossi, C. Hierold, A. Stemmer, W. J. Stark, Angew. Chem. Int. Ed. 2009, 48, 224.

[29] Y. Lu, B. R. Goldsmith, N. J. Kybert, A. T. C. Johnson, Appl. Phys. Lett. 2010, 97, 083107.

[30] Y. Cui, S. N. Kim, S. E. Jones, L. L. Wissler, R. R. Naik, M. C. McAlpine, Nano Lett. 2010, 10, 4559.

[31] J. Katoch, S. N. Kim, Z. F. Kuang, B. L. Farmer, R. R. Nalk, S. A. Tatulian, M. Ishigami, Nano Lett. 2012, 12, 2342.
[32] Z. X. Zhang, H. L. Huang, X. M. Yang, L. Zang, J. Phys. Chem. Lett. 2011, 2, 2897

[33] Q. Y. He, S. X. Wu, Z. Y. Yin, H. Zhang, Chem. Sci. 2012, 3, 1764.

[34] S. X. Wu, Q. Y. He, C. L. Tan, Y. D. Wang, H. Zhang, Small 2013, 9, 1160 .

[35] C. T. Lin, P. T. K. Loan, T. Y. Chen, K. K. Liu, C. H. Chen, K. H. Wei, L. J. Li, Adv. Funct. Mater. 2013, 23, 2301.

[36] S. Chen, Z. B. Zhang, L. P. Ma, P. Ahlberg, X. D. Gao, Z. J. Qiu, D. P. Wu, W. C. Ren, H. M. Cheng, S. L. Zhang, Appl. Phys. Lett. 2012, 101, 154106.

[37] W. Fu, C. Nef, A. Tarasov, M. Wipf, R. Stoop, O. Knopfmacher, M. Weiss, M. Calame, C. Schonenberger, Nanoscale 2013, 5, 12104

[38] J. Yang, M. Z. Ma, L. Q. Li, Y. F. Zhang, W. Huang, X. C. Dong, Nanoscale 2014, 6, 13301.

[39] a) J. L. Xia, F. Chen, J. H. Li, N. J. Tao, Nat. Nanotechnol. 2009, 4 505; b) S. Luryi, Appl. Phys. Lett. 1988, 52, 501.

[40] J. O. Bockris, E. Gileadi, K. Muller, J. Chem. Phys. 1966, 44, 1445.

[41] V. M. Aguilella, J. Pellicer, M. Aguilella-Arzo, Langmuir 1999, 15, 6156

[42] P. Bergveld, Sens. Actuat. B-Chem. 2003, 88, 1.

[43] P. Bergveld, IEEE Trans. Biomed. Eng. 1970, Bm17, 70

[44] J. J. Gooding, Small 2006, 2, 313.

[45] X. P. A. Gao, G. F. Zheng, C. M. Lieber, Nano Lett. 2010, 10, 547.

[46] G. S. Kulkarni, Z. H. Zhong, Nano Lett. 2012, 12, 719.

[47] K. S. Chang, C. C. Chen, J. T. Sheu, Y. K. Li, Sens. Actuat. B-Chem. 2009, 138, 148.

[48] V. Singh, D. Joung, L. Zhai, S. Das, S. I. Khondaker, S. Seal, Prog. Mater. Sci. 2011, 56, 1178.

[49] a) E. W. Hill, A. Vijayaragahvan, K. Novoselov, IEEE Sens. J. 2011, 11, 3161; b) H. C. Cheng, R. J. Shiue, C. C. Tsai, W. H. Wang, Y. T. Chen, ACS Nano 2011, 5, 2051.

[50] S. M. Sze, K. K. Ng, Physics of Semiconductor Devices, 3rd Edition 2007, 832pp.

[51] D. H. Tien, J.-Y. Park, K. B. Kim, N. Lee, T. Choi, P. Kim, T. Taniguchi, K. Watanabe, Y. Seo, ACS Appl. Mater. Interfaces 2016 8, 3072.

[52] G. Auton, J. Zhang, R. K. Kumar, H. Wang, X. Zhang, Q. Wang, E. Hill, A. Song, Nat. Commun. 2016, 7, 11670.

[53] G. Liu, S. L. Rumyantsev, C. Jiang, M. S. Shur, A. A. Balandin, IEEE Electron Device L 2015, 36, 1202

[54] K. S. Kim, Y. Zhao, H. Jang, S. Y. Lee, J. M. Kim, K. S. Kim, J. H. Ahn, P. Kim, J. Y. Choi, B. H. Hong, Nature 2009, 457, 706.

[55] X. S. Li, W. W. Cai, J. H. An, S. Kim, J. Nah, D. X. Yang, R. Piner, A. Velamakanni, I. Jung, E. Tutuc, S. K. Banerjee, L. Colombo, R. S. Ruoff, Science 2009, 324, 1312.

[56] A. W. Tsen, L. Brown, M. P. Levendorf, F. Ghahari, P. Y. Huang, R. W. Havener, C. S. Ruiz-Vargas, D. A. Muller, P. Kim, J. Park Science 2012, 336, 1143.

[57] a) SIGMA-ALDRICH, Graphene field effect transistor chip, 2016, http://www.sigmaaldrich.com/technical-documents/articles/materials-science/graphene-field-effect-transistors.html\#newMaterials; b) L. BGT Materials Limited, Grat-FET ${ }^{\mathrm{TM}}, 2015$, http://bgtmaterials.com/uploads/BGT\%20Materials\%20Limited_Grat\%20FET_ Data\%20Sheet\%2017Jun2015.pdf.

[58] H. S. Song, S. L. Li, H. Miyazaki, S. Sato, K. Hayashi, A. Yamada, N. Yokoyama, K. Tsukagoshi, Sci. Rep-UK 2012, 2, 337.

[59] a) Z. Yan, Z. W. Peng, J. M. Tour, Acc. Chem. Res. 2014, 47, 1327; b) T. R. Wu, X. F. Zhang, Q. H. Yuan, J. C. Xue, G. Y. Lu, Z. H. Liu, H. S. Wang, H. M. Wang, F. Ding, Q. K. Yu, X. M. Xie, M. H. Jiang, Nat. Mater. 2016, 15, 43.

[60] a) K. H. Lee, H. J. Shin, J. Lee, I. Y. Lee, G. H. Kim, J. Y. Choi, S. W. Kim, Nano Lett. 2012, 12, 714; b) N. Petrone, C. R. Dean, I. Meric, A. M. van der Zande, P. Y. Huang, L. Wang, D. Muller, K. L. Shepard, J. Hone, Nano Lett. 2012, 12, 2751. 
[61] L. Banszerus, M. Schmitz, S. Engels, J. Dauber, M. Oellers, F. Haupt, K. Watanabe, T. Taniguchi, B. Beschoten, C. Stampfer, Sci. Adv. 2015, 1, e1500222.

[62] F. N. Hooge, T. G. M. Kleinpenning, L. K. J. Vandamme, Rep. Prog. Phys. 1981, 44, 479.

[63] a) N. K. Rajan, D. A. Routenberg, M. A. Reed, Appl. Phys. Lett. 2011, 98, 264107; b) A. Tarasov, W. Fu, O. Knopfmacher, J. Brunner, M. Calame, C. Schonenberger, Appl. Phys. Lett. 2011, 98, 012114; c) K. Bedner, V. A. Guzenko, A. Tarasov, M. Wipf, R. L. Stoop, D. Just, S. Rigante, W. Y. Fu, R. A. Minamisawa, C. David, M. Calame, J. Gobrecht, C. Schonenberger, Sens. Mater. 2013, 25, 567.

[64] G. X. Liu, S. Rumyantsev, M. S. Shur, A. A. Balandin, Appl. Phys. Lett. 2013, 102, 093111.

[65] B. Veigas, E. Fortunato, P. V. Baptista, Sensors-Basel 2015, 15, 10380.

[66] B. B. Zhan, C. Li, J. Yang, G. Jenkins, W. Huang, X. C. Dong, Small 2014, 10, 4042

[67] O. Moldovan, B. Iniguez, M. J. Deen, L. F. Marsal, IET Circ. Device Syst. 2015, 9, 446.

[68] L. H. Hess, M. Seifert, J. A. Garrido, P IEEE 2013, 101, 1780.

[69] G. Liu, W. Stillman, S. Rumyantsev, Q. Shao, M. Shur, A. A. Balandin, Appl. Phys. Lett. 2009, 95, 033103.

[70] M. A. Stolyarov, G. X. Liu, S. L. Rumyantsev, M. Shur, A. A. Balandin, Appl. Phys. Lett. 2015, 107, 023106.

[71] N. K. Rajan, K. Brower, X. X. Duan, M. A. Reed, Appl. Phys. Lett. 2014, 104, 084106.

[72] S. Sorgenfrei, C. Y. Chiu, M. Johnston, C. Nuckolls, K. L. Shepard, Nano Lett. 2011, 11, 3739.

[73] P. Debye, E. Hückel, Phys. Z. 1923, 24, 185.

[74] E. Stern, R. Wagner, F. J. Sigworth, R. Breaker, T. M. Fahmy, M. A. Reed, Nano Lett. 2007, 7, 3405.

[75] G. J. Zhang, G. Zhang, J. H. Chua, R. E. Chee, E. H. Wong, A. Agarwal, K. D. Buddharaju, N. Singh, Z. Q. Gao, N. Balasubramanian, Nano Lett. 2008, 8, 1066.

[76] N. Gao, W. Zhou, X. C. Jiang, G. S. Hong, T. M. Fu, C. M. Lieber, Nano Lett. 2015, 15, 2143.

[77] S. Mukherjee, X. Meshik, M. Choi, S. Farid, D. Datta, Y. Lan, S. Poduri, K. Sarkar, U. Baterdene, C. E. Huang, Y. Y. Wang, P. Burke, M. Dutta, M. A. Stroscio, IEEE T. Nanobiosci. 2015, 14, 967.

[78] D. J. Kim, I. Y. Sohn, J. H. Jung, O. J. Yoon, N. E. Lee, J. S. Park, Biosens. Bioelectron. 2013, 41, 621.

[79] N. Clement, K. Nishiguchi, J. F. Dufreche, D. Guerin, A. Fujiwara, D. Vuillaume, Appl. Phys. Lett. 2011, 98, 014104.

[80] E. Stern, A. Vacic, N. K. Rajan, J. M. Criscione, J. Park, B. R. Ilic, D. J. Mooney, M. A. Reed, T. M. Fahmy, Nat. Nanotechnol. 2010, $5,138$.

[81] J. T. Robinson, J. S. Burgess, C. E. Junkermeier, S. C. Badescu, T. L. Reinecke, F. K. Perkins, M. K. Zalalutdniov, J. W. Baldwin, J. C. Culbertson, P. E. Sheehan, E. S. Snow, Nano Lett. 2010, 10, 3001.

[82] J. S. Burgess, B. R. Matis, J. T. Robinson, F. A. Bulat, F. Keith Perkins, B. H. Houston, J. W. Baldwin, Carbon 2011, 49, 4420.

[83] F. Withers, M. Dubois, A. K. Savchenko, Phys. Rev. B 2010, 82, 073403.

[84] K.-I. Ho, C.-H. Huang, J.-H. Liao, W. Zhang, L.-J. Li, C.-S. Lai, C.-Y. Su, Sci. Rep-UK 2014, 4, 5893.

[85] X. Zhang, A. Hsu, H. Wang, Y. Song, J. Kong, M. S. Dresselhaus, T. Palacios, ACS Nano 2013, 7, 7262.

[86] B. Li, L. Zhou, D. Wu, H. Peng, K. Yan, Y. Zhou, Z. Liu, ACS Nano 2011, 5, 5957

[87] A. Wei, J. Wang, Q. Long, X. Liu, X. Li, X. Dong, W. Huang, Mater. Res. Bull. 2011, 46, 2131.
[88] H. Feng, R. Cheng, X. Zhao, X. Duan, J. Li, Nat. Commun. 2013, 4, 1539.

[89] X. Dong, Q. Long, A. Wei, W. Zhang, L.-J. Li, P. Chen, W. Huang, Carbon 2012, 50, 1517.

[90] H. Zhang, E. Bekyarova, J.-W. Huang, Z. Zhao, W. Bao, F. Wang, R. C. Haddon, C. N. Lau, Nano Lett. 2011, 11, 4047.

[91] X. C. Dong, D. L. Fu, W. J. Fang, Y. M. Shi, P. Chen, L. J. Li, Small 2009, 5, 1422.

[92] Q. H. Wang, M. C. Hersam, Nat. Chem. 2009, 1, 206.

[93] a) Y. Zhu, Y. Hao, E. A. Adogla, J. Yan, D. Li, K. Xu, Q. Wang, J. Hone, Q. Lin, Nanoscale 2016, 8, 5815; b) F. M. Koehler, N. A. Luechinger, D. Ziegler, E. K. Athanassiou, R. N. Grass, A. Rossi, C. Hierold, A. Stemmer, W. J. Stark, Angew. Chem. Int. Ed. 2009, 48, 224; c) T. F. v. Dijkman, Vol. Copper trispyrazolylborate complexes for ethene detection, Doctoral Thesis, Leiden University, 2016. Retrieved from http://hdl.handle.net/1887/39518.

[94] C. Li, K. Komatsu, S. Bertrand, G. Clavé, S. Campidelli, A. Filoramo, S. Guéron, H. Bouchiat, Phys. Rev. B 2016, 93, 045403.

[95] X. Wang, J.-B. Xu, W. Xie, J. Du, J. Phys. Chem. C 2011, 115, 7596.

[96] Y. Y. Wang, P. J. Burke, Nano Res. 2014, 7, 1650.

[97] N. Dontschuk, A. Stacey, A. Tadich, K. J. Rietwyk, A. Schenk, M. T. Edmonds, O. Shimoni, C. I. Pakes, S. Prawer, J. Cervenka, Nat. Commun. 2015, 6, 6563.

[98] N. S. Green, M. L. Norton, Anal. Chim. Acta 2015, 853, 127.

[99] N. Mohanty, V. Berry, Nano Lett. 2008, 8, 4469.

[100] A. R. Gao, N. L. Zou, P. F. Dai, N. Lu, T. Li, Y. L. Wang, J. L. Zhao, H. J. Mao, Nano Lett. 2013, 13, 4123.

[101] C.-T. Lin, P. T. K. Loan, T.-Y. Chen, K.-K. Liu, C.-H. Chen, K.-H. Wei, L.-). Li, Adv. Funct. Mater. 2013, 23, 2301.

[102] a) J. Wang, H. Zhu, Y. Xu, W. Yang, A. Liu, F. Shan, M. Cao, J. Liu, Sens. Actuat. B-Chem. 2015, 220, 1186; b) Q. Guo, H. Zhu, F. Liu, A. Y. Zhu, J. C. Reed, F. Yi, E. Cubukcu, ACS Photonics 2014, 1, 221; c) M. B. Lerner, F. Matsunaga, G. H. Han, S. J. Hong, J. Xi, A. Crook, J. M. Perez-Aguilar, Y. W. Park, J. G. Saven, R. Liu, A. T. C. Johnson, Nano Lett. 2014, 14, 2709; d) S. Eissa, C. Tlili, L. L'Hocine, M. Zourob, Biosens. Bioelectron. 2012, 38, 308.

[103] L. Feng, Y. Chen, J. Ren, X. Qu, Biomaterials 2011, 32, 2930.

[104] a) L. Feng, L. Wu, J. Wang, J. Ren, D. Miyoshi, N. Sugimoto, X. Qu, Adv. Mater. 2012, 24, 125; b) H. Zhang, Y. Wang, D. Zhao, D. Zeng, J. Xia, A. Aldalbahi, C. Wang, L. San, C. Fan, X. Zuo, X. Mi, ACS Appl. Mater. Interfaces 2015, 7, 16152.

[105] a) S. Li, X. Zhong, H. Yang, Y. Hu, F. Zhang, Z. Niu, W. Hu, Z. Dong, J. Jin, R. Li, J. Ma, Carbon 2011, 49, 4239; b) L.-M. Lu, H.-B. Li, F. Qu, X.-B. Zhang, G.-L. Shen, R.-Q. Yu, Biosens. Bioelectron. 2011, 26, 3500; c) J. Luo, S. Jiang, H. Zhang, J. Jiang, X. Liu, Anal. Chim. Acta 2012, 709, 47.

[106] B. C. Zhong, M. A. Uddin, A. Singh, R. Webb, G. Koley, Appl. Phys. Lett. 2016, 108, 093102.

[107] F. Jimenez-Villacorta, E. Climent-Pascual, R. Ramirez-Jimenez, J. Sanchez-Marcos, C. Prieto, A. de Andrés, Carbon 2016, 101, 305

[108] K. S. Novoselov, D. Jiang, F. Schedin, T. J. Booth, V. V. Khotkevich, S. V. Morozov, A. K. Geim, Proc. Natl. Acad. Sci. USA 2005, 102, 10451.

[109] V. Georgakilas, M. Otyepka, A. B. Bourlinos, V. Chandra, N. Kim, K. C. Kemp, P. Hobza, R. Zboril, K. S. Kim, Chem. Rev. 2012, 112, 6156.

[110] F. Yan, M. Zhang, J. H. Li, Adv. Healthcare Mater. 2014, 3, 313.

[111] H. Tanveer, P. Puspamitra, A. Rajeev, Nanotechnology 2014, 25, 325501.

[112] T. Hussain, P. Panigrahi, R. Ahuja, Phys. Chem. Chem. Phys. 2014, 16,8100

[113] A. Y. S. Eng, Z. Sofer, P. Šimek, J. Kosina, M. Pumera, Chem. Eur. J. 2013, 19, 15583

[114] K. K. Tadi, S. Pal, T. N. Narayanan, Sci. Rep-UK 2016, 6, 25221. 
[115] X. Chia, A. Ambrosi, M. Otyepka, R. Zbořil, M. Pumera, Chem. Eur. J. 2014, 20, 6665.

[116] V. Urbanová, K. Holá, A. B. Bourlinos, K. Čépe, A. Ambrosi, A. H. Loo, M. Pumera, F. Karlický, M. Otyepka, R. Zbořil, Adv. Mater. 2015, 27, 2305.

[117] H. P. Boehm, A. Clauss, U. Hofmann, G. O. Fischer, Z. Naturforsch. Pt B 1962, B17, 150.

[118] D. A. Dikin, S. Stankovich, E. J. Zimney, R. D. Piner G. H. B. Dommett, G. Evmenenko, S. T. Nguyen, R. S. Ruoff, Nature 2007, 448, 457.

[119] a) S. Seo, M. Min, J. Lee, T. Lee, S. Y. Choi, H. Lee, Angew. Chem. Int. Ed. 2012, 51, 108; b) S. Borini, R. White, D. Wei, M. Astley, S. Haque, E. Spigone, N. Harris, J. Kivioja, T. Ryhanen, ACS Nano 2013, 7, 11166; c) X. H. Zhang, Y. Zhang, Q. L. Liao, Y. Song, S. W. Ma, Small 2013, 9, 4045.

[120] F. Karlicky, K. K. R. Datta, M. Otyepka, R. Zboril, ACS Nano 2013 7, 6434.

[121] a) D. E. Jiang, B. G. Sumpter, S. Dai, J. Phys. Chem. B 2006, 110, 23628; b) R. K. Shervedani, A. Amini, N. Sadeghi, Biosens. Bioelectron. 2016, 77, 478; c) C. S. Rao Vusa, V. Manju, S. Berchmans, P. Arumugam, RSC Adv. 2016, 6, 33409; d) M. Mooste, E. Kibena, J. Kozlova, M. Marandi, L. Matisen, A. Niilisk, V. Sammelselg, K. Tammeveski, Electrochim. Acta 2015, 161, 195.

[122] F. M. Koehler, A. Jacobsen, K. Ensslin, C. Stampfer, W. J. Stark, Small 2010, 6, 1125 .

[123] Q. H. Wang, Z. Jin, K. K. Kim, A. J. Hilmer, G. L. C. Paulus, C. J. Shih, M. H. Ham, J. D. Sanchez-Yamagishi, K. Watanabe, T. Taniguchi, J. Kong, P. Jarillo-Herrero, M. S. Strano, Nat. Chem. 2012, 4, 724 .

[124] G. L. C. Paulus, Q. H. Wang, M. S. Strano, Acc. Chem. Res. 2013, 46, 160.

[125] A. Bellunato, H. Arjmandi Tash, Y. Cesa, G. F. Schneider, Chem. Phys. Chem. 2016, 17, 785.

[126] A. Sinitskii, A. Dimiev, D. A. Corley, A. A. Fursina, D. V. Kosynkin, J. M. Tour, ACS Nano 2010, 4, 1949.

[127] a) S. D. Chakarova-Kack, E. Schroder, B. I. Lundqvist, D. C. Langreth, Phys. Rev. Lett. 2006, 96, 146107; b) W. Orellana, J. D. Correa, J. Mater. Sci. 2015, 50, 898.

[128] G. F. Schneider, Q. Xu, S. Hage, S. Luik, J. N. H. Spoor, S. Malladi, H. Zandbergen, C. Dekker, Nat. Commun. 2013, 4, 2619

[129] W. Sun, Y. Lu, Y. Wu, Y. Zhang, P. Wang, Y. Chen, G. Li, Sens. Actuat. B-Chem. 2014, 202, 160.

[130] a) F. K. Perkins, A. L. Friedman, E. Cobas, P. M. Campbell, G. G. Jernigan, B. T. Jonker, Nano Lett. 2013, 13, 668; b) B. Cho, M. G. Hahm, M. Choi, J. Yoon, A. R. Kim, Y. J. Lee, S. G. Park, J. D. Kwon, C. S. Kim, M. Song, Y. Jeong, K. S. Nam, S. Lee, T. J. Yoo, C. G. Kang, B. H. Lee, H. C. Ko, P. M. Ajayan, D. H. Kim, Sci. Rep-UK 2015, 5, 8052.

[131] a) L. Y. Feng, L. Wu, J. S. Wang, J. S. Ren, D. Miyoshi, N. Sugimoto, X. G. Qu, Adv. Mater. 2012, 24, 125; b) J. P. Wang, B. J. Zou, J. Z. Rui, Q. X. Song, T. Kajiyama, H. Kambara, G. H. Zhou, Microchim. Acta 2015, 182, 1095

[132] a) X. S. Li, Y. W. Zhu, W. W. Cai, M. Borysiak, B. Y. Han, D. Chen, R. D. Piner, L. Colombo, R. S. Ruoff, Nano Lett. 2009, 9, 4359, b) L. B. Gao, G. X. Ni, Y. P. Liu, B. Liu, A. H. C. Neto, K. P. Loh, Nature 2014, 505, 190; c) Y. D. Su, H. L. Han, Q. Cai, Q. Wu, M. X. Xie, D. Y. Chen, B. S. Geng, Y. B. Zhang, F. Wang, Y. R. Shen, C. S. Tian, Nano Lett. 2015, 15, 6501

[133] a) W. H. Lin, T. H. Chen, J. K. Chang, J. I. Taur, Y. Y. Lo, W. L. Lee, C. S. Chang, W. B. Su, C. I. Wu, ACS Nano 2014, 8 , 1784; b) I. Pasternak, A. Krajewska, K. Grodecki, I. Jozwik-Biala, K. Sobczak, W. Strupinski, AIP Adv. 2014, 4, 097133; c) G. H. Zhang, A. G. Guell, P. M. Kirkman, R. A. Lazenby T. S. Miller, P. R. Unwin, ACS Appl. Mater. Inter. 2016, 8, 8008.

[134] a) A. Q. Zhang, C. M. Lieber, Chem. Rev. 2016, 116, 215; b) S. Choi, H. Lee, R. Ghaffari, T. Hyeon, D. H. Kim, Adv. Mater. 2016, 28, 4203.

[135] C. Mackin, T. Palacios, Analyst 2016, 141, 2704.

[136] a) D. M. Engelman, Nature 2005, 438, 578; b) K. Murata, K. Mitsuoka, T. Hirai, T. Walz, P. Agre, J. B. Heymann, A. Engel, Y. Fujiyoshi, Nature 2000, 407, 599; c) Z. L. Li, H. M. Ding, Y. Q. Ma, J. Phys. Condens. Matter. 2016, 28; d) X. Y. Zhang, W. Y. Fu, C. G. Palivan, W. Meier, Sci. Rep-UK 2013, 3, 2196.

[137] a) A. V. Titov, P. Kral, R. Pearson, ACS Nano 2010, 4, 229; b) J. Mao, R. H. Guo, L. T. Yan, Biomaterials 2014, 35, 6069; c) T. Yue, X. Wang, X. Zhang, F. Huang, RSC Adv. 2015, 5, 30092; d) J. J. Liu, M. M. Bao, J. L. Li, Y. Y. Weng, Y. J. Dou, B. Yuan, K. Yang, Y. Q. Ma, Carbon 2016, 98, 300.

[138] M. Hirtz, A. Oikonomou, T. Georgiou, H. Fuchs, A. Vijayaraghavan, Nat. Commun. 2013, 4, 2591.

[139] D. R. Dreyer, S. Park, C. W. Bielawski, R. S. Ruoff, Chem. Soc. Rev. 2010, 39, 228

[140] a) Y. S. Tu, M. Lv, P. Xiu, T. Huynh, M. Zhang, M. Castelli, Z. R. Liu, Q. Huang, C. H. Fan, H. P. Fang, R. H. Zhou, Nat. Nanotechnol. 2013, 8, 594; b) V. T. H. Pham, V. K. Truong, M. D. J. Quinn S. M. Notley, Y. Guo, V. A. Baulin, M. Al Kobaisi, R. J. Crawford, E. P. Ivanova, ACS Nano 2015, 9, 8458

[141] S. H. Li, A. J. Stein, A. Kruger, R. M. Leblanc, J. Phys. Chem. C 2013, $117,16150$.

[142] H. Kim, Y. R. Jang, J. Yoo, Y. S. Seo, K. Y. Kim, J. S. Lee, S. D. Park, C. J. Kim, J. Koo, Langmuir 2014, 30, 2170.

[143] R. Frost, G. E. Jonsson, D. Chakarov, S. Svedhem, B. Kasemo, Nano Lett. 2012, 12, 3356.

[144] a) R. Frost, S. Svedhem, C. Langhammer, B. Kasemo, Langmuir 2016, 32, 2708; b) H. Lei, X. Zhou, H. Wu, Y. Song, J. Hu, S. Guo, Y. Zhang, Langmuir 2014, 30, 4678.

[145] a) S. Bae, H. Kim, Y. Lee, X. F. Xu, J. S. Park, Y. Zheng, J. Balakrishnan, T. Lei, H. R. Kim, Y. I. Song, Y. J. Kim, K. S. Kim, B. Ozyilmaz, J. H. Ahn, B. H. Hong, S. lijima, Nat. Nanotechnol. 2010, 5, 574; b) J. U. Park, S. Nam, M. S. Lee, C. M. Lieber, Nat. Mater. 2012, 11, 120.

[146] P. Nguyen, V. Berry, J. Phys. Chem. Lett. 2012, 3, 1024

[147] R. Kempaiah, A. Chung, V. Maheshwari, ACS Nano 2011, 5, 6025.

[148] A. Fabbro, D. Scaini, V. Leon, E. Vazquez, G. Cellot, G. Privitera, L. Lombardi, F. Torrisi, F. Tomarchio, F. Bonaccorso, S. Bosi, A. C. Ferrari, L. Ballerini, M. Prato, ACS Nano 2016, 10, 615.

[149] a) H. Z. Lei, X. J. Zhou, H. X. Wu, Y. Song, J. Hu, S. W. Guo, Y. Zhang, Langmuir 2014, 30, 4678; b) Y. X. Liu, X. C. Dong, P. Chen, Chem. Soc. Rev. 2012, 41, 2283.

[150] P. K. Ang, M. Jaiswal, C. H. Y. X. Lim, Y. Wang, J. Sankaran, A. Li, C. T. Lim, T. Wohland, B. Ozyilmaz, K. P. Loh, ACS Nano 2010, 4 7387.

[151] Y. Y. Wang, T. D. Pham, K. Zand, J. Li, P. J. Burke, ACS Nano 2014, 8, 4228.

[152] M. Peplow, Nature 2015, 522, 268.

[153] W. G. Huang, A. K. Diallo, J. L. Dailey, K. Besar, H. E. Katz, J. Mater. Chem. C 2015, 3, 6445

[154] C. Z. Liao, M. Zhang, L. Y. Niu, Z. J. Zheng, F. Yan, J. Mater. Chem. B 2014, 2, 191.

[155] P.-G. Su, H.-C. Shieh, Sens. Actuat. B-Chem. 2014, 190, 865.

[156] A. K. Singh, M. A. Uddin, J. T. Tolson, H. Maire-Afeli, N. Sbrockey, G. S. Tompa, M. G. Spencer, T. Vogt, T. S. Sudarshan, G. Koley, Appl. Phys. Lett. 2013, 102, 043101.

[157] F. Schedin, A. K. Geim, S. V. Morozov, E. W. Hill, P. Blake, M. I. Katsnelson, K. S. Novoselov, Nat. Mater. 2007, 6, 652 
[158] Y. Dan, Y. Lu, N. J. Kybert, Z. Luo, A. T. C. Johnson, Nano Lett. 2009, 9, 1472.

[159] Y.-R. Li, S.-h. Chang, C.-T. Chang, W.-L. Tsai, Y.-K. Chiu, P.-Y. Yang, H.-C. Cheng, Jpn. J. Appl. Phys. 2016, 55, 04EM08.

[160] Y. Zhu, C. Wang, N. Petrone, J. Yu, C. Nuckolls, J. Hone, Q. Lin, Appl. Phys. Lett. 2015, 106, 123503.

[161] P. K. Ang, W. Chen, A. T. S. Wee, K. P. Loh, J. Am. Chem. Soc. 2008, 130, 14392.

[162] Y. Ohno, K. Maehashi, Y. Yamashiro, K. Matsumoto, Nano Lett. 2009, 9, 3318.

[163] Z. Cheng, Q. Li, Z. Li, Q. Zhou, Y. Fang, Nano Lett. 2010, 10, 1864.

[164] K. Maehashi, Y. Sofue, S. Okamoto, Y. Ohno, K. Inoue, K. Matsumoto, Sens. Actuat. B-Chem. 2013, 187, 45.

[165] S. Yasuyuki, O. Yasuhide, M. Kenzo, I. Koichi, M. Kazuhiko, Jpn. J. Appl. Phys. 2011, 50, 06GE07.

[166] H. G. Sudibya, Q. He, H. Zhang, P. Chen, ACS Nano 2011, 5, 1990.

[167] T. Zhang, Z. Cheng, Y. Wang, Z. Li, C. Wang, Y. Li, Y. Fang, Nano Lett. 2010, 10, 4738.

[168] C. Wang, X. Cui, Y. Li, H. Li, L. Huang, J. Bi, J. Luo, L. Q. Ma, W. Zhou, Y. Cao, B. Wang, F. Miao, Sci. Rep-UK 2016, 6, 21711.

[169] Z. Yin, Q. He, X. Huang, J. Zhang, S. Wu, P. Chen, G. Lu, P. Chen, Q. Zhang, Q. Yan, H. Zhang, Nanoscale 2012, 4, 293.

[170] D.-J. Kim, H.-C. Park, I. Y. Sohn, J.-H. Jung, O. J. Yoon, J.-S. Park, M.-Y. Yoon, N.-E. Lee, Small 2013, 9, 3352.

[171] Y. Ohno, K. Maehashi, K. Matsumoto, J. Am. Chem. Soc. 2010, 132, 18012.

[172] K. Vasu, S. Sridevi, S. Sampath, A. Sood, Sensor Actuat. B-Chem. 2015, 221, 1209.

[173] A. A. Cagang, I. H. Abidi, A. Tyagi, J. Hu, F. Xu, T. J. Lu, Z. Luo, Anal. Chim. Acta 2016, 917, 101.

[174] C. Liao, C. Mak, M. Zhang, H. L. Chan, F. Yan, Adv. Mater. 2015, $27,676$.

[175] M. Zhang, C. Liao, C. H. Mak, P. You, C. L. Mak, F. Yan, Sci. Rep-UK 2015, 5, 8311.

[176] T. Cohen-Karni, Q. Qing, Q. Li, Y. Fang, C. M. Lieber, Nano Lett. 2010, 10, 1098.

[177] L. H. Hess, M. Jansen, V. Maybeck, M. V. Hauf, M. Seifert, M. Stutzmann, I. D. Sharp, A. Offenhäusser, J. A. Garrido, Adv. Mater. 2011, 23, 5045

[178] M. Ishigami, J. H. Chen, W. G. Cullen, M. S. Fuhrer, E. D. Williams, Nano Lett. 2007, 7, 1643

[179] G. S. Kulkarni, K. Reddy, Z. H. Zhong, X. D. Fan, Nat. Commun. 2014, 5, 4376.

[180] H. Vedala, D. C. Sorescu, G. P. Kotchey, A. Star, Nano Lett. 2011, 11, 2342.

[181] M. Nakamura, Y. Kanai, Y. Ohno, K. Maehashi, K. Inoue, K. Matsumoto, Jpn. J. Appl. Phys. 2015, 54, 6S1.

[182] C. Toumazou, P. Georgiou, P. Bergveld, Electron. Lett. 2011, 47, S7.

[183] a) X. B. Tan, H. J. Chuang, M. W. Lin, Z. X. Zhou, M. M. C. Cheng, J. Phys. Chem. C 2013, 117, 27155; b) S. S. Kwon, J. Yi, W. W. Lee, J. H. Shin, S. H. Kim, S. H. Cho, S. Nam, W. I. Park, ACS Appl. Mater. Inter. 2016, 8, 834.

[184] L. Larrimore, S. Nad, X. J. Zhou, H. Abruna, P. L. McEuen, Nano Lett. 2006, 6, 1329.

[185] K. Maehashi, Y. Sofue, S. Okamoto, Y. Ohno, K. Inoue, K. Matsumoto, Sensor Actuat. B-Chem. 2013, 187, 45.

[186] Z. Y. Yang, N. N. Dai, R. T. Lu, Z. H. Huang, F. Y. Kang, New Carbon Mater. 2015, 30, 511.

[187] J. B. Chang, G. H. Zhou, E. R. Christensen, R. Heideman, J. H. Chen, Anal. Bioanal. Chem. 2014, 406, 3957.

[188] T. Zhang, Z. G. Cheng, Y. B. Wang, Z. J. Li, C. X. Wang, Y. B. Li, Y. Fang, Nano Lett. 2010, 10, 4738.
[189] C. Y. Wang, X. Y. Cui, Y. Li, H. B. Li, L. Huang, J. Bi, J. Luo, L. Q. Ma, W. Zhou, Y. Cao, B. G. Wang, F. Miao, Sci. Rep-UK 2016, 6, 21711.

[190] a) H. G. Sudibya, Q. Y. He, H. Zhang, P. Chen, ACS Nano 2011, 5, 1990; b) G. H. Zhou, J. B. Chang, S. M. Cui, H. H. Pu, Z. H. Wen, J. H. Chen, ACS Appl. Mater. Inter. 2014, 6, 19235.

[191] a) M. S. Mannoor, H. Tao, J. D. Clayton, A. Sengupta, D. L. Kaplan, R. R. Naik, N. Verma, F. G. Omenetto, M. C. McAlpine, Nat. Commun. 2012, 3, 763; b) D. A. Deen, E. J. Olson, M. A. Ebrish, S. J. Koester, IEEE Sens. J. 2014, 14, 1459; c) X. J. Huang, T. Leng, M. J. Zhu, X. Zhang, J. C. Chen, K. Chang, M. Aqeeli, A. K. Geim, K. S. Novoselov, Z. R. Hu, Sci. Rep-UK 2015, 5, 18298.

[192] H. Huang, L. Tao, F. Liu, L. Ji, Y. Hu, M. M.-C. Cheng, P.-Y. Chen, D. Akinwande, Microsyst. Nanoeng. 2016, 2, 16018.

[193] S. Mao, J. B. Chang, G. H. Zhou, J. H. Chen, Small 2015, 11, 5336.

[194] S. Viswanathan, T. N. Narayanan, K. Aran, K. D. Fink, J. Paredes, P. M. Ajayan, S. Filipek, P. Miszta, H. C. Tekin, F. Inci, U. Demirci, P. Li, K. I. Bolotin, D. Liepmann, V. Renugopalakrishanan, Mater. Today 2015, 18, 513.

[195] W. L. Clarke, D. Cox, L. A. Gonderfrederick, W. Carter, S. L. Pohl, Diabetes Care 1987, 10, 622.

[196] M. Zhang, C. Z. Liao, C. H. Mak, P. You, C. L. Mak, F. Yan, Sci. Rep-UK 2015, 5, 8311

[197] Y. H. Kwak, D. S. Choi, Y. N. Kim, H. Kim, D. H. Yoon, S.-S. Ahn, J.-W. Yang, W. S. Yang, S. Seo, Biosens. Bioelectron. 2012, 37, 82.

[198] X. You, J. J. Pak, Sens. Actuat. B-Chem. 2014, 202, 1357.

[199] A. A. Cagang, I. H. Abidi, A. Tyagi, J. Hu, F. Xu, T. J. Lu, Z. T. Luo, Anal. Chim. Acta 2016, 917, 101

[200] C. Z. Liao, M. Zhang, L. Y. Niu, Z. J. Zheng, F. Yan, J. Mater. Chem. B 2013, 1, 3820.

[201] X. C. Dong, Y. M. Shi, W. Huang, P. Chen, L. J. Li, Adv. Mater. 2010, 22, 1649

[202] a) R. Q. Li, Q. Li, Y. Zhou, K. P. Chen, C. X. Zhang, L. Chen, L. Gao, J. J. Cui, Mater. Technol. 2015, 30, B163; b) S. J. Heerema, C. Dekker, Nat. Nanotechnol. 2016, 11, 127; c) H. Arjmandi-Tash, L. A. Belyaeva, G. F. Schneider, Chem. Soc. Rev. 2016, 45, 476.

[203] F. Traversi, C. Raillon, S. M. Benameur, K. Liu, S. Khlybov, M. Tosun, D. Krasnozhon, A. Kis, A. Radenovic, Nat. Nanotechnol. 2013, 8, 939

[204] a) D. R. Mills, R. L. Peterson, S. Spiegelm, Proc. Natl. Acad. Sci. USA 1967, 58, 217; b) K. Xu, J. Qian, P. Shukla, M. Dutta, M. A. Stroscio, 2012 15th International Workshop on Computational Electronics (Iwce) 2012.

[205] D. J. Kim, H. C. Park, I. Y. Sohn, J. H. Jung, O. J. Yoon, J. S. Park, M. Y. Yoon, N. E. Lee, Small 2013, 9, 3352.

[206] C. Reiner-Rozman, C. Kotlowski, W. Knoll, Biosensors (Basel) 2016, 6, 17.

[207] J. M. Rothberg, W. Hinz, T. M. Rearick, J. Schultz, W. Mileski, M. Davey, J. H. Leamon, K. Johnson, M. J. Milgrew, M. Edwards, J. Hoon, J. F. Simons, D. Marran, J. W. Myers, J. F. Davidson, A. Branting, J. R. Nobile, B. P. Puc, D. Light, T. A. Clark, M. Huber, J. T. Branciforte, I. B. Stoner, S. E. Cawley, M. Lyons, Y. T. Fu, N. Homer, M. Sedova, X. Miao, B. Reed, J. Sabina, E. Feierstein, M. Schorn, M. Alanjary, E. Dimalanta, D. Dressman, R. Kasinskas, T. Sokolsky, J. A. Fidanza, E. Namsaraev, K. J. McKernan, A. Williams, G. T. Roth, J. Bustillo, Nature 2011, 475, 348.

[208] J. L. Her, T. M. Pan, W. Y. Lin, K. S. Wang, L. J. Li, Sens. Actuat. B-Chem. 2013, 182, 396.

[209] X. J. Duan, R. X. Gao, P. Xie, T. Cohen-Karni, Q. Qing, H. S. Choe, B. Z. Tian, X. C. Jiang, C. M. Lieber, Nat. Nanotechnol. 2012, 7, 174.

[210] L. H. Hess, M. Jansen, V. Maybeck, M. V. Hauf, M. Seifert, M. Stutzmann, I. D. Sharp, A. Offenhausser, J. A. Garrido, Adv. Mater. 2011, 23, 5045. 
[211] a) M. D. Halbach, U. Egert, J. Hescheler, K. Banach, Cell Physiol. Biochem. 2003, 13, 271; b) F. Heer, S. Hafizovic, T. Ugniwenko, U. Frey, W. Franks, E. Perriard, J. C. Perriard, A. Blau, C. Ziegler, A. Hierlemann, Biosens. Bioelectron. 2007, 22, 2546; c) S. Ingebrandt, C. K. Yeung, M. Krause, A. Offenhausser, Biosens. Bioelectron. 2001, 16, 565

[212] O. P. Hamill, A. Marty, E. Neher, B. Sakmann, F. J. Sigworth, Pflug. Arch. Eur. J. Phy. 1981, 391, 85.

[213] C. Becker-Freyseng, P. Fromherz, EPL-Europhys. Lett. 2011, 96, 38005.

[214] a) S. J. Park, O. S. Kwon, S. H. Lee, H. S. Song, T. H. Park, J. Jang Nano Lett. 2012, 12, 5082; b) J. H. An, S. J. Park, O. S. Kwon, J. Bae, J. Jang, ACS Nano 2013, 7, 10563; c) B. K. Sharma, J. H. Ahn, Solid State Electron. 2013, 89, 177.

[215] a) S. M. Choi, S. H. Jhi, Y. W. Son, Phys. Rev. B 2010, 81, 081407; b) H. H. P. Garza, E. W. Kievit, G. F. Schneider, U. Staufer, Nano Lett. 2014, 14, 4107.

[216] Y. Y. Shao, J. Wang, H. Wu, J. Liu, I. A. Aksay, Y. H. Lin, Electroanal. 2010, 22, 1027.

[217] a) Y. Wang, Y. Shao, D. W. Matson, J. Li, Y. Lin, ACS Nano 2010, 4, 1790; b) J. Guo, T. Zhang, C. Hu, L. Fu, Nanoscale 2015, 7, 1290 c) V. Urbanová, F. Karlický, A. Matěj, F. Šembera, Z. Janoušek, J. A. Perman, V. Ranc, K. Čépe, J. Michl, M. Otyepka, Nanoscale 2016, 8, 12134.

[218] O. Akhavan, E. Ghaderi, R. Rahighi, ACS Nano 2012, 6, 2904.

[219] R. L. McCreery, Chem. Rev. 2008, 108, 2646.

[220] a) C. Ruan, W. Shi, H. Jiang, Y. Sun, X. Liu, X. Zhang, Z. Sun, L. Dai, D. Ge, Sensor Actuat. B-Chem. 2013, 177, 826; b) B. Unnikrishnan, S. Palanisamy, S.-M. Chen, Biosens. Bioelectron. 2013, 39, 70.

[221] S. S. J. Aravind, T. T. Baby, T. Arockiadoss, R. B. Rakhi, S. Ramaprabhu, Thin Solid Films 2011, 519, 5667.

[222] a) Y. Guo, Y. Guo, C. Dong, Electrochim. Acta 2013, 113, 69; b) K.-J. Huang, Y.-J. Liu, H.-B. Wang, T. Gan, Y.-M. Liu, L.-L. Wang, Sensor Actuat. B-Chem. 2014, 191, 828; c) Y. Ni, P. Wang, H. Song, X. Lin, S. Kokot, Anal. Chim. Acta 2014, 821, 34.

[223] L. Zhu, L. Luo, Z. Wang, Biosens. Bioelectron. 2012, 35, 507.

[224] a) B. Jin, P. Wang, H. Mao, B. Hu, H. Zhang, Z. Cheng, Z. Wu, X. Bian, C. Jia, F. Jing, Biosens. Bioelectron. 2014, 55, 464; b) F. Liu, K. S. Choi, T. J. Park, S. Y. Lee, T. S. Seo, BioChip J. 2011, 5, 123 c) Z. Wang, F. Li, J. Xia, L. Xia, F. Zhang, S. Bi, G. Shi, Y. Xia, J. Liu, Y. Li, Biosens. Bioelectron. 2014, 61, 391.

[225] Y. Wang, Z. Li, D. Hu, C.-T. Lin, J. Li, Y. Lin, J. Am. Chem. Soc. 2010 132, 9274

[226] A. Muthurasu, V. Ganesh, Appl. Biochem. Biotechnol. 2014, 174, 945.

[227] a) J. C. Claussen, A. Kumar, D. B. Jaroch, M. H. Khawaja, A. B. Hibbard, D. M. Porterfield, T. S. Fisher, Adv. Funct. Mater. 2012, 22, 3399; b) D. Pan, Y. Gu, H. Lan, Y. Sun, H. Gao, Anal.
Chim. Acta 2015, 853, 297; c) Z. Yang, Y. Cao, J. Li, Z. Jian, Y. Zhang, X. Hu, Anal. Chim. Acta 2015, 871, 35.

[228] Y. Yang, M. Kang, S. Fang, M. Wang, L. He, J. Zhao, H. Zhang, Z. Zhang, Sens. Actuat. B-Chem 2015, 214, 63.

[229] W. Yuan, Y. Zhou, Y. Li, C. Li, H. Peng, J. Zhang, Z. Liu, L. Dai, G. Shi, Sci. Rep-UK 2013, 3, 2248.

[230] W. Li, C. Tan, M. A. Lowe, H. D. Abruna, D. C. Ralph, ACS Nano 2011, 5, 2264

[231] S. Banerjee, J. Shim, J. Rivera, X. Jin, D. Estrada, V. Solovyeva, X. You, J. Pak, E. Pop, N. Aluru, ACS Nano 2012, 7, 834

[232] a) C. Tan, J. n. Rodríguez-López, J. J. Parks, N. L. Ritzert, D. C. Ralph, H. C. D. Abruña, ACS Nano 2012, 6, 3070; b) N. L. Ritzert, J. Rodríguez-López, C. Tan, H. C. D. Abruña, Langmuir 2013, 29, 1683; c) A. G. Guell, A. S. Cuharuc, Y. R. Kim, G. H. Zhang, S. Y. Tan, N. Ebejer, P. R. Unwin, ACS Nano 2015, 9, 3558.

[233] J.-H. Zhong, J. Zhang, X. Jin, J.-Y. Liu, Q. Li, M.-H. Li, W. Cai, D.-Y. Wu, D. Zhan, B. Ren, J. Am. Chem. Soc. 2014, 136, 16609 .

[234] a) R. Sharma, J. H. Baik, C. J. Perera, M. S. Strano, Nano Lett. 2010, 10, 398; b) K. S. Mali, J. Greenwood, J. Adisoejoso R. Phillipson, S. De Feyter, Nanoscale 2015, 7, 1566; c) K. Yong-Jin, K. Yuna, N. Konstantin, H. Byung Hee, 2D Materials 2015, 2, 042001; d) A. Criado, M. Melchionna, S. Marchesan, M. Prato Angew. Chem. Int. Ed. 2015, 54, 10734.

[235] W. Y. Fu, L. Y. Feng, D. Mayer, G. Panaitov, D. Kireev A. Offenhausser, H. J. Krause, Nano Lett. 2016, 16, 2295.

[236] a) P. Bergveld, Biosens. Bioelectron. 1991, 6, 55; b) P. Bergveld, Sens. Actuat. A-Phys 1996, 56, 65; c) B. K. Wunderlich, P. A. Neff, A. R. Bausch, Appl. Phys. Lett. 2007, 91, 083904.

[237] A. Tarasov, M. Wipf, K. Bedner, J. Kurz, W. Fu, V. A. Guzenko, O. Knopfmacher, R. L. Stoop, M. Calame, C. Schonenberger, Langmuir 2012, 28, 9899.

[238] O. Knopfmacher, A. Tarasov, W. Y. Fu, M. Wipf, B. Niesen, M. Calame, C. Schonenberger, Nano Lett. 2010, 10, 2268.

[239] R. L. Stoop, M. Wipf, S. Mueller, K. Bedner, I. A. Wright, C. J. Martin, E. C. Constable, W. Y. Fu, A. Tarasov, M. Calame, C. Schonenberger, Sens. Actuat. B-Chem. 2015, 220, 500.

[240] a) L. Liao, Y. C. Lin, M. Q. Bao, R. Cheng, J. W. Bai, Y. A. Liu, Y. Q. Qu, K. L. Wang, Y. Huang, X. F. Duan, Nature 2010, 467, 305; b) Y. Q. Wu, K. A. Jenkins, A. Valdes-Garcia, D. B. Farmer, Y. Zhu, A. A. Bol, C. Dimitrakopoulos, W. J. Zhu, F. N. Xia, P. Avouris Y. M. Lin, Nano Lett. 2012, 12, 3062

[241] W. Fu, M. El Abbassi, T. Hasler, M. Jung, M. Steinacher M. Calame, C. Schonenberger, G. Puebla-Hellmann, S. Hellmuller, T. Ihn, A. Wallraff, Appl. Phys. Lett. 2014, 104, 013102.

[242] R. Pethig, Biomicrofluidics 2010, 4, 022811.

[243] H. J. Lee, J. G. Yook, Biosens. Bioelectron. 2014, 61, 448. 